\title{
Adversarial domain translation networks enable fast and accurate large-scale atlas-level single-cell data integration
}

Jia Zhao ${ }^{1 *}$ Gefei Wang ${ }^{*}$, Jingsi Ming ${ }^{2}$, Zhixiang Lin $^{3}$, Yang Wang ${ }^{1}$ Tabula Microcebus Consortium, Angela Ruohao $\mathrm{Wu}^{4,5 \dagger}$, Can Yang ${ }^{1 \dagger}$

${ }^{1}$ Department of Mathematics, The Hong Kong University of Science and Technology, Hong Kong SAR, China

${ }^{2}$ Academy of Statistics and Interdisciplinary Sciences, KLATASDS-MOE, East China Normal University, Shanghai, China

${ }^{3}$ Department of Statistics, The Chinese University of Hong Kong, Hong Kong SAR, China

${ }^{4}$ Division of Life Science, The Hong Kong University of Science and Technology, Hong Kong SAR, China

${ }^{5}$ Department of Chemical and Biological Engineering, The Hong Kong University of Science and Technology, Hong Kong SAR, China

\section{Abstract}

The rapid emergence of large-scale atlas-level single-cell RNA-sequencing (scRNA-seq) datasets from various sources presents remarkable opportunities for broad and deep biological investigations through integrative analyses. However, harmonizing such datasets requires integration approaches to be not only computationally scalable, but also capable of preserving a wide range of fine-grained cell populations. We created Portal, a unified framework of adversarial domain translation to learn harmonized representations of datasets. With innovation in model and algorithm designs, Portal achieves superior performance in preserving biological variation during integration, while having significantly reduced running time and memory compared to existing approaches, achieving integration of millions of cells in minutes with low memory consumption. We demonstrate the efficiency and accuracy of Portal using diverse datasets ranging from

*These authors contributed to this work equally.

†Correspondence: angelawu@ust.hk, macyang@ust.hk. 
mouse brain atlas projects, the Tabula Muris project, and the Tabula Microcebus project.

Portal has broad applicability and in addition to integrating multiple scRNA-seq datasets, it can also integrate scRNA-seq with single-nucleus RNA-sequencing (snRNA-seq) data. Finally, we demonstrate the utility of Portal by applying it to the integration of cross-species datasets with limited shared-information between them, and are able to elucidate biological insights into the similarities and divergences in the spermatogenesis process between mouse, macaque, and human.

\section{Introduction}

Advances in single-cell sequencing have enabled identification of novel cell types [1, 2], investigation of gene regulation networks [3, 4], and understanding of cellular differentiation processes [5, 6]. As single-cell technologies rapidly evolved over recent years, its experimental throughput substantially increased, allowing researchers to profile increasingly complex and diverse samples, and accelerating the accumulation of vast numbers of rich datasets over time [7, 8, 9]. Integrative and comparative analyses of such large-scale datasets originating from various samples, different platforms and data types, as well as across multiple species, offer unprecedented opportunities to establish a comprehensive picture of diverse cellular behaviors. Integration is a critical step, to account for heterogeneity of different data sources when taking advantage of single-cell data from different studies [10]. Thus, integration methods that can efficiently and accurately harmonize a wide range of data types are essential for accelerating life sciences research [11].

Although integration methods for single-cell transcriptomics analysis have evolved along with single-cell sequencing technologies, the rapid accumulation of new and diverse single-cell datasets has introduced three major challenges to the integration task. First, as the sample size of each single-cell dataset grows dramatically, numerous extensive datasets with hundreds of thousands or even millions of cells have been produced [8, 9, 12]. The emergence of large-scale datasets requires integration methods to be fast, memory-efficient, and scalable to millions of cells. Second, technology now allows effective, comprehensive characterization of complex organs, containing rare subpopulations of cells that can now be captured, albeit in small numbers, thanks to the scale of profiling that is now possible [7, 13]. Investigation into highlevel heterogeneity among cell populations is essential for understanding the mechanism of 
complex biological systems. Hence, the ideal integration method needs to carefully preserve finegrained cell populations from each atlas-level dataset. Third, the biological origins of datasets has expanded in diversity, with data now spanning across not only different technological platforms and data types, different individual donors, but even across different species, which can be especially interesting for evolutionary studies [14, 15, 16]. Integrative analysis of such diverse datasets would allow researchers to unify resources to address a wider range of biological questions. Recent single-cell atlasing efforts are a primary example of these challenges - various human tissue atlases [12, 17], mouse multi-tissue atlases [7, 18], and non-human primate atlases [19, 20] have been generated, culminating in data from millions of single cells and single nuclei. Both within and across atlas comparisons are of interest. To perform integrative and comparative analyses based on such diverse data sources, there is an urgent need for methods that can flexibly account for heterogeneous dataset-specific effects, while maintaining a high level of integration accuracy.

Many methods have been developed to align single-cell datasets [10], including Harmony [21], Seurat [22], online iNMF [23], fastMNN [24], Scanorama [25] and BBKNN [26]. Several of these methods that were designed for large datasets at the time of publication are now less attractive in terms of scalability in the face of atlas-level dataset sizes. For instance, a representative category of methods leverages the mutual nearest neighbors (MNN) to perform data alignment. These MNN-based methods, such as Seurat, fastMNN and Scanorama, require identification of MNN pairs across datasets, thus the time and memory costs quickly become unbearably high when the dataset exceeds one million cells. Another limitation of existing methods is that they are mainly targeted towards integrating datasets of less complex tissues, utilizing strategies such as MNN, matrix factorization, and soft-clustering to capture major biological variations. With these strategies, inaccurate mixing of different cell types can be avoided when clear clustering patterns are present; but when dealing with more complex tissues, they tend to overcorrect fine-grained cell subpopulations, resulting in the loss of power in revealing interesting biological variations. Lastly, most existing methods are designed to correct batch effects caused by technical artifacts. To this end, a number of methods, like BBKNN and fastMNN, assume that the biological variation is much larger than the variation of batch effects. This assumption may not be true when applied across data types and species.

To simultaneously address the above three challenges, we created Portal, a machine learning- 
based algorithm for aligning atlas-level single-cell datasets with high efficiency, flexibility, and accuracy. Viewing datasets from different studies as distinct domains with domain-specific effects (including technical variation and other sources of unwanted variation), Portal achieves extraordinary data alignment performance through a unified framework of domain translation networks that incorporates an adversarial learning mechanism [27]. To find the correspondence between two domains, our domain translation network utilizes an encoder to embed cells from one domain into a latent space where domain-specific effects are removed, and then uses a generator to map latent codes to another domain. The generator simulates the generation process of domain-specific effects. In each domain, a discriminator is trained to identify where poor alignment between the distributions of original cells and transferred cells occurs. The feedback signal from the discriminator is used to strengthen the domain translation network for better alignment. The nonlinearity of encoders and generators in the adversarial domain translation framework enables Portal to account for complex domain-specific effects. In contrast to existing domain translation methods [28, 29, 30], Portal has the following unique features. First, Portal has a uniquely designed discriminator which can adaptively distinguish domainshared cell types and domain-unique cell types. Therefore, Portal will not force the alignment of domain-unique cell types, avoiding the risk of overcorrection. Second, without using any cell type label information, three regularizers of Portal can guide domain translation networks to find correct correspondence between domains, account for domain-specific effects, and retain biological variation in the latent space. Third, through a tailored design of light-weight neural networks and mini-batch optimization accelerated by graphics processing units (GPUs), Portal can scale up to datasets containing millions of cells in minutes with nearly constant memory usage. With the above innovations in model and algorithm designs, Portal enables fast and accurate integration of atlas-level datasets across samples, technological platforms, data types, and species.

Through integration of heterogeneous collections of atlas-level single-cell RNA sequencing (scRNA-seq) data, Portal shows its superiority over state-of-the-art alignment algorithms in terms of both computational efficiency and accuracy. We then show that Portal can accurately align cells from complex tissues profiled by scRNA-seq and single-nucleus RNA sequencing (snRNA-seq), and also perform cross-species alignment of the gradient of cells in the spermatogenesis process, demonstrating Portal's versatility and power for a broad range of 
applications. Comprehensive analyses of real, expert annotated data confirm that integrated cell embeddings provided by Portal can be reliably used for identification of rare cell populations via clustering or label transfer, studies of differentiation trajectories, and transfer learning across data types and across species. Portal is now publicly available as a Python package (https://github.com/YangLabHKUST/Portal), serving as an efficient, reliable and flexible tool for integrative analyses.

\section{Results}

\section{Method Overview: Portal learns a harmonized representation of dif- ferent datasets with adversarial domain translation.}

Expression measurements from different datasets fall into different domains due to the existence of domain-specific effects, including technical variation and other sources of unwanted variation (Fig. 1a), causing difficulty when performing joint analyses. Without loss of generality, here we consider two domains, $\mathcal{X}$ and $\mathcal{Y}$. We assume that domain $\mathcal{X}$ and domain $\mathcal{Y}$ can be connected through a low-dimensional shared latent space $\mathcal{Z}$, which captures the biological variation and is not affected by the domain-specific effects. By taking the measurements of cells from $\mathcal{X}$ and $\mathcal{Y}$ as inputs, we aim to learn a harmonized representation of cells in latent space $\mathcal{Z}$ to obtain data alignment between $\mathcal{X}$ and $\mathcal{Y}$.

We achieve the above goal through a unified framework of adversarial domain translation, namely "Portal". Domains and the shared latent space are connected by encoders and generators (Fig. 1 $\mathbf{b}$ ). Encoder $E_{1}(\cdot): \mathcal{X} \rightarrow \mathcal{Z}$ is designed to remove the domain-specific effects when mapping cells from $\mathcal{X}$ into $\mathcal{Z}$, and generator $G_{1}(\cdot): \mathcal{Z} \rightarrow \mathcal{X}$ is designed to simulate the domain-specific effects when mapping cells from $\mathcal{Z}$ into $\mathcal{X}$. By symmetry, encoder $E_{2}(\cdot): \mathcal{Y} \rightarrow \mathcal{Z}$ and generator $G_{2}(\cdot): \mathcal{Z} \rightarrow \mathcal{Y}$ are designed with the same role in connecting $\mathcal{Y}$ and $\mathcal{Z}$. To transfer cells between $\mathcal{Y}$ and $\mathcal{X}$ through shared latent space $\mathcal{Z}$ (Fig. $1 \mathbf{b}$ ), encoder $E_{2}(\cdot)$ and generator $G_{1}(\cdot)$ work together to form one domain translation network $G_{1}\left(E_{2}(\cdot)\right): \mathcal{Y} \rightarrow \mathcal{Z} \rightarrow \mathcal{X}$. Clearly, encoder $E_{1}(\cdot)$ and generator $G_{2}(\cdot)$ form another domain translation network $G_{2}\left(E_{1}(\cdot)\right): \mathcal{X} \rightarrow \mathcal{Z} \rightarrow \mathcal{Y}$. To achieve the mixing of original cells and transferred cells, discriminators $D_{1}(\cdot)$ and $D_{2}(\cdot)$ are deployed in domains $\mathcal{X}$ and $\mathcal{Y}$ to identify where poor mixing occurs (Fig. 1 1 ). The discriminators' feedback then guides the domain translation networks to improve the mixing. 
However, the well mixing of original cells and transferred cells in each domain does not imply extraordinary data alignment across domains. First, a domain-unique cell population should not be mixed with cells from another domain. Second, cell types $A$ and $B$ in domain $\mathcal{X}$ could be incorrectly aligned with cell types $B$ and $A$ in domain $\mathcal{Y}$, respectively, although the distributions of original cells and transferred cells are well mixed. To address these issues, Portal has the following unique features, which distinguishes it from existing adversarial domain translation frameworks [28, 29]. On one hand, we deploy the tailored design of discriminators $D_{1}(\cdot)$ and $D_{2}(\cdot)$ such that they can distinguish domain-unique cell types from cell types shared across different domains. The domain-unique cell types will be treated as outliers and left in the discriminator's inactive region (Fig. 11c). In such a way, these cell types will not be enforced for alignment, avoiding the risk of overcorrection. On the other hand, we design three regularizers to find correct correspondence across domains and avoid incorrect alignment when the distributions are well mixed.

Specifically, let $\mathbf{x}$ and $\mathbf{y}$ be the samples from domains $\mathcal{X}$ and $\mathcal{Y}$, respectively. We consider the following framework of adversarial domain translation,

$$
\begin{aligned}
\min _{\left\{E_{1}, G_{1}, E_{2}, G_{2}\right\}\left\{D_{1}, D_{2}\right\}} & \mathcal{L}_{\mathcal{X}}\left(D_{1}, E_{2}, G_{1}\right)+\mathcal{L}_{\mathcal{Y}}\left(D_{2}, E_{1}, G_{2}\right), \\
\text { subject to } & \mathcal{R}_{\mathrm{AE}}\left(E_{1}, G_{1}, E_{2}, G_{2}\right) \leq t_{\mathrm{AE}}, \\
& \mathcal{R}_{\mathrm{LA}}\left(E_{1}, G_{1}, E_{2}, G_{2}\right) \leq t_{\mathrm{LA}} \\
& \mathcal{R}_{\mathrm{cos}}\left(E_{1}, G_{1}, E_{2}, G_{2}\right) \leq t_{\mathrm{cos}} .
\end{aligned}
$$

In model (1), $\mathcal{L}_{\mathcal{X}}\left(D_{1}, E_{2}, G_{1}\right):=\mathbb{E}\left[\log D_{1}(\mathbf{x})\right]+\mathbb{E}\left[\log \left(1-D_{1}\left(G_{1}\left(E_{2}(\mathbf{y})\right)\right)\right)\right]$ and $\mathcal{L}_{\mathcal{Y}}\left(D_{2}, E_{1}, G_{2}\right):=$ $\mathbb{E}\left[\log D_{2}(\mathbf{y})\right]+\mathbb{E}\left[\log \left(1-D_{2}\left(G_{2}\left(E_{1}(\mathbf{x})\right)\right)\right)\right]$ are the objective functions for adversarial learning of domain translation networks $G_{1}\left(E_{2}(\cdot)\right)$ and $G_{2}\left(E_{1}(\cdot)\right)$ in $\mathcal{X}$ and $\mathcal{Y}$, respectively. Discriminators $D_{1}(\cdot)$ and $D_{2}(\cdot)$ are trained to distinguish between "real" cells (i.e. original cells in a domain), and "fake" cells (i.e. transferred cells generated by domain translation networks) by minimizing $\mathcal{L}_{\mathcal{X}}+\mathcal{L}_{\mathcal{Y}}$, while the domain translation networks are trained against the discriminators by maximizing $\mathcal{L}_{\mathcal{X}}+\mathcal{L}_{\mathcal{Y}}$. These three regularizers $\mathcal{R}_{\mathrm{AE}}, \mathcal{R}_{\mathrm{LA}}$ and $\mathcal{R}_{\mathrm{cos}}$ play a critical role in finding correct correspondence of cells between two domains, accounting for domain-specific effects, and retaining biological variation in the latent space (Fig. 1 d). More specifically, the first regularizer $\mathcal{R}_{\mathrm{AE}}:=\frac{1}{p}\left\{\mathbb{E}\left[\left\|\mathbf{x}-G_{1}\left(E_{1}(\mathbf{x})\right)\right\|_{2}^{2}\right]+\mathbb{E}\left[\left\|\mathbf{y}-G_{2}\left(E_{2}(\mathbf{y})\right)\right\|_{2}^{2}\right]\right\}$, where $p$ is the dimensionality of domains $\mathcal{X}$ and $\mathcal{Y}$, requires the autoencoder consistency in domains $\mathcal{X}$ and $\mathcal{Y}$; the second regularizer $\mathcal{R}_{\mathrm{LA}}:=\frac{1}{q}\left\{\mathbb{E}\left[\left\|E_{1}(\mathbf{x})-E_{2}\left(G_{2}\left(E_{1}(\mathbf{x})\right)\right)\right\|_{2}^{2}\right]+\mathbb{E}\left[\left\|E_{2}(\mathbf{y})-E_{1}\left(G_{1}\left(E_{2}(\mathbf{y})\right)\right)\right\|_{2}^{2}\right]\right\}$ 
where $q$ is the dimensionality of $\mathcal{Z}$, imposes the consistency constraint in the latent space; and the third regularizer $\mathcal{R}_{\cos }:=\mathbb{E}\left[1-\frac{<\mathbf{x}, G_{2}\left(E_{1}(\mathbf{x})\right)>}{\|\mathbf{x}\|_{2}\left\|G_{2}\left(E_{1}(\mathbf{x})\right)\right\|_{2}}\right]+\mathbb{E}\left[1-\frac{<\mathbf{y}, G_{1}\left(E_{2}(\mathbf{y})\right)>}{\|\mathbf{y}\|_{2}\left\|G_{1}\left(E_{2}(\mathbf{y})\right)\right\|_{2}}\right]$ introduces the cross-domain correspondence by preserving the cosine similarity between a sample and its transferred version; $t_{\mathrm{AE}}, t_{\mathrm{LA}}$ and $t_{\mathrm{cos}}$ are their corresponding constraint parameters. More detailed explanation can be found in the Method section.

We solve the above optimization problem via alternating updates by stochastic gradient descent. The algorithm is extremely computationally efficient with the support of stochastic optimization accelerated by GPUs. After the training process, Portal learns a harmonized representation of different domains in shared latent space $\mathcal{Z}$. Samples from $\mathcal{X}$ and $\mathcal{Y}$ can be transferred into latent space $\mathcal{Z}$ to form an integrated dataset $\left\{E_{1}(\mathbf{x})\right\}_{\mathbf{x} \in \mathcal{X}} \cup\left\{E_{2}(\mathbf{y})\right\}_{\mathbf{y} \in \mathcal{Y}}$ using encoders $E_{1}(\cdot)$ and $E_{2}(\cdot)$, facilitating the downstream integrative analysis of cross-domain single-cell datasets.

\section{Accurate integration of atlas-level datasets within minutes and re- quiring lower memory consumption compared to other methods.}

The rapid accumulation of large-scale single-cell datasets requires integration algorithms to efficiently handle datasets containing millions of cells without loss of accuracy. For a comprehensive comparison, we first benchmarked Portal against multiple methods, including Harmony [21], Seurat v3 [22], online iNMF [23], fastMNN [24], Scanorama [25] and BBKNN [26], in terms of integration performance. Using massive scRNA-seq datasets from diverse tissue types with curated cell cluster annotations, including mouse spleen, marrow, and bladder [7], we quantitatively evaluated the integration performance of each method. We evaluated alignment performance, which can sometimes be interpreted as batch effects removal performance, using k-nearest neighbor batch-effect test (kBET) [32]; the higher the kBET score, the higher the degree of mixing across datasets. We also assessed cluster identity preservation performance using the adjust rand index (ARI) and average silhouette width (ASW) metrics. Using the authors' cell type annotations as ground truth, higher ARI and ASW scores denote that correct cell type identities are preserved after integration, while lower scores indicate inappropriate merging of cell types during integration. Based on these metrics, we found that in general, fastMNN, Scanorama, and BBKNN have less satisfactory integration performance compared to the other four methods (Figs. 2, S3 and S4): as indicated by the relatively lower kBET scores 
a
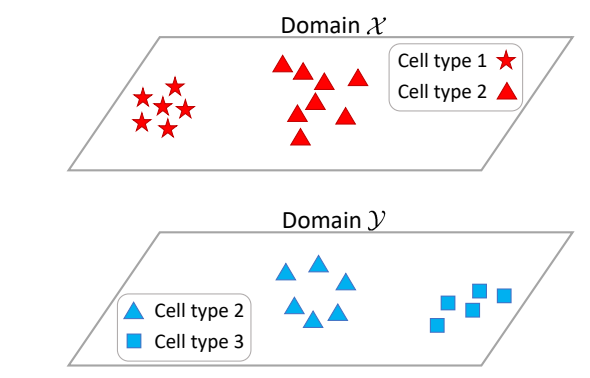

C
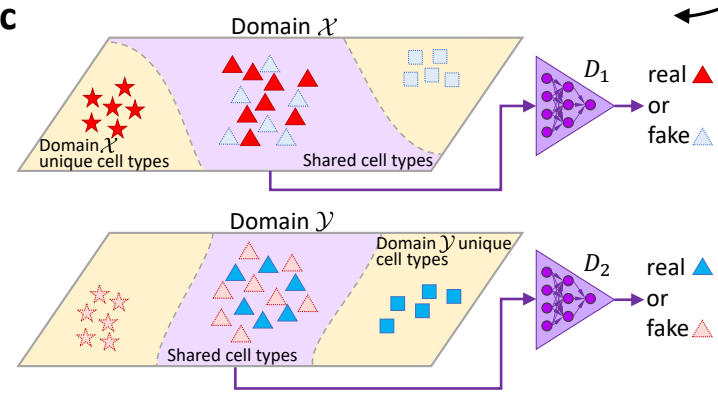

Discriminator active region boundary

Active region

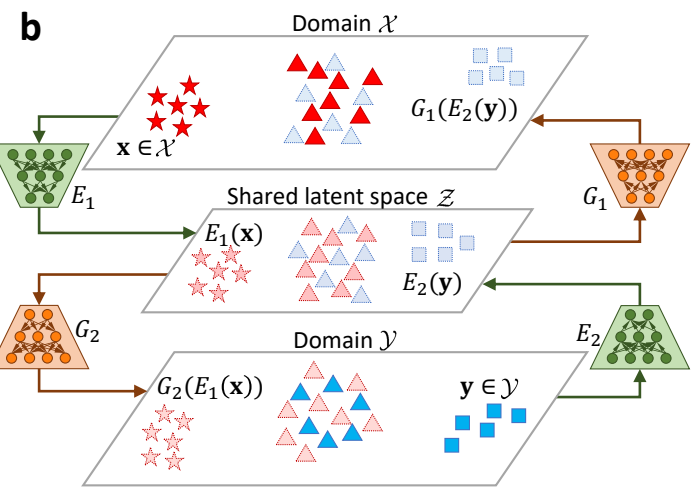

d

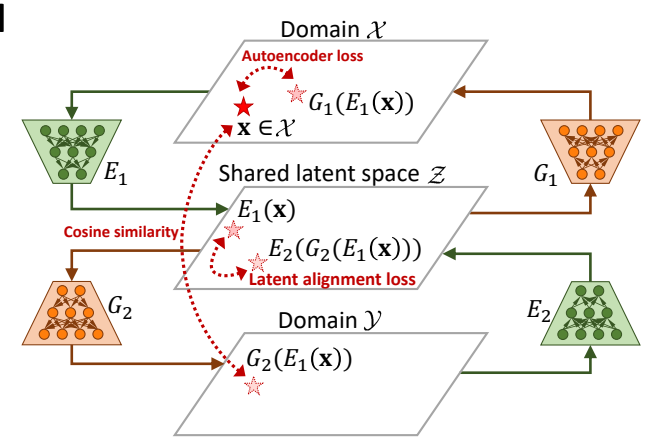

Figure 1: Overview of Portal. a. Portal regards different single-cell datasets as different domains. Joint analyses of these datasets are confounded by domain-specific effects, representing the unwanted technical variation. b. Portal employs encoders $E_{1}(\cdot), E_{2}(\cdot)$ to embed the biological variation of domains $\mathcal{X}$ and $\mathcal{Y}$ into a shared latent space $\mathcal{Z}$, where domain-specific effects are removed. The generating process of domain-specific effects are captured by two generators $G_{1}(\cdot)$ and $G_{2}(\cdot)$. Encoder $E_{1}(\cdot)$ and generator $G_{2}(\cdot)$ form a domain translation network $G_{2}\left(E_{1}(\cdot)\right)$ mapping from $\mathcal{X}$ to $\mathcal{Y}$; Encoder $E_{2}(\cdot)$ and generator $G_{1}(\cdot)$ form another domain translation network mapping from $\mathcal{Y}$ to $\mathcal{X}$. c. Encoders and generators are trained by competing against specially designed discriminators $D_{1}(\cdot)$ and $D_{2}(\cdot)$. In each domain, a discriminator is trained to distinguish between original cells in this domain and cells transferred from another domain, providing feedback signals to assist alignment. To prevent overcorrection of domain-unique cell types, the discriminators in Portal with the tailored design are also able to distinguish between domain-unique cell types and domain-shared cell types. With this design, Portal can focus only on merging cells of high probability to be of domain-shared cell types, while it remains inactive on cells of domain-unique cell types. d. Portal leverages three regularizers to help it find correct and consistent correspondence across domains, including the autoencoder regularizer, the latent alignment regularizer and the cosine similarity regularizer.

of these three methods, we found that observable batch effects still exist in the integration results they produced (Fig. 2a); in addition, their ARI and ASW metrics are also lower (Fig. $2 \mathrm{~b})$.

Among those methods with high user popularity, Harmony, Seurat, and online iNMF 

available under aCC-BY-NC-ND 4.0 International license.

a
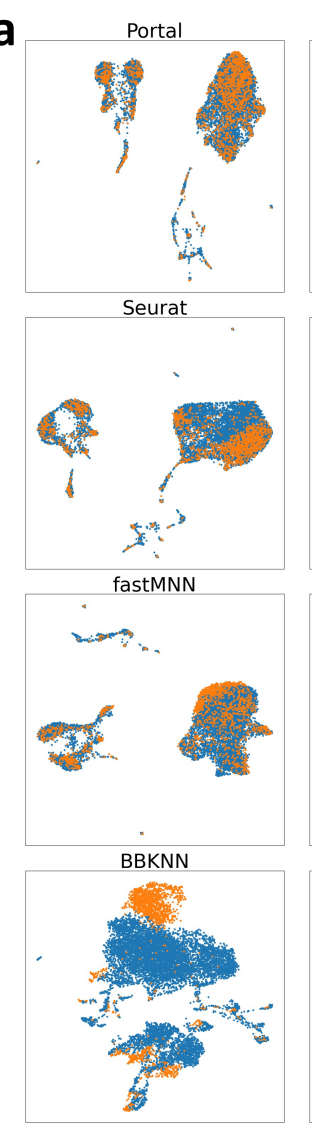
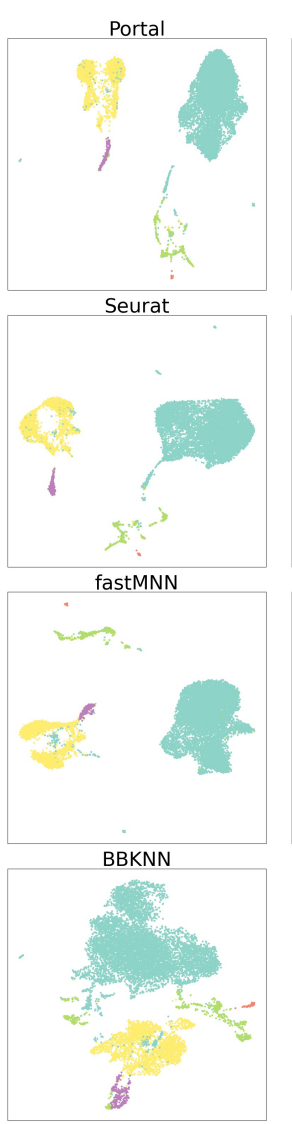
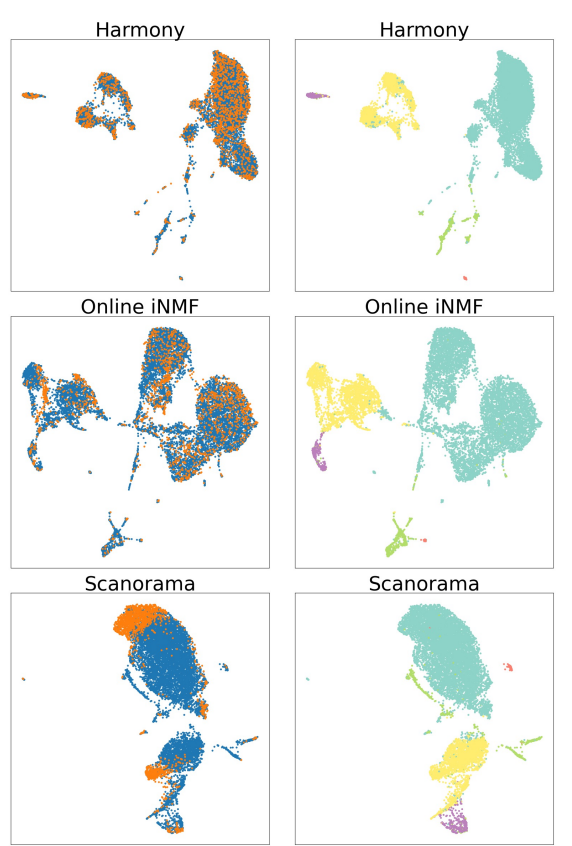

Method Cell type

$\begin{array}{llll}-10 \mathrm{X} & \text { B cell }\end{array}$

- SS2 - Dendritic cell

- Macrophage

- Natural killer cell

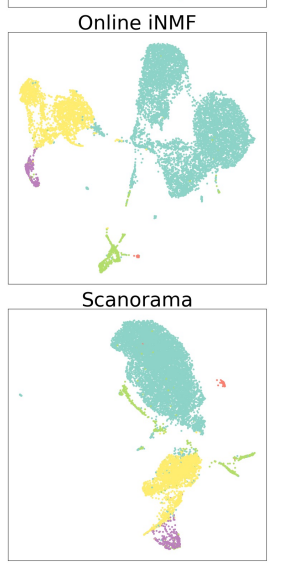

T cell b
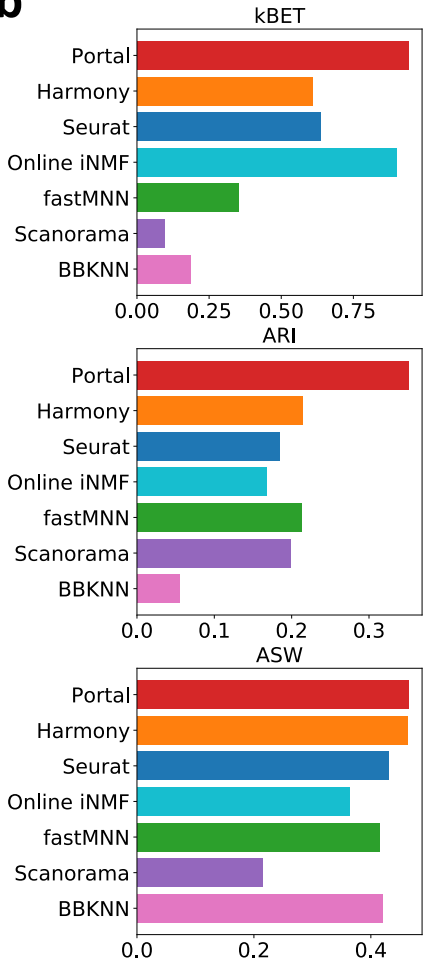

Figure 2: Comparison of integration methods based on mouse spleen data. We integrated mouse spleen scRNA-seq datasets profiled by 10X Genomics (10X) and SMART-seq2 (SS2). a. UMAP [31] plots colored by profiling methods and cell types. b. Alignment (kBET) and cluster preservation performance (ARI and ASW) of compared methods evaluated on the mouse spleen data.

also showed the best alignment performance results. To offer precise and robust integration performance, Seurat [22] utilizes the detection of mutual nearest neighbors (MNN) to build correspondence between datasets in the shared embedding space obtained by applying canonical correlation analysis (CCA). Harmony 21] learns a simple linear correction for dataset-specific effects by running an iterative soft clustering algorithm, enabling fast computation on large datasets. Online iNMF [23] is a recently developed approach based on widely used integration method LIGER [33]. It extends LIGER's non-negative matrix factorization to an iterative and incremental version to improve its scalability, while it has nearly the same performance as LIGER. For the remainder of this study, we focus our discussion on comparisons between Portal and these three high-performing and popular methods (Fig. 3) in the main text. The comparisons with other methods are provided in Supplementary Information (Fig. S5). 

available under aCC-BY-NC-ND 4.0 International license.

a

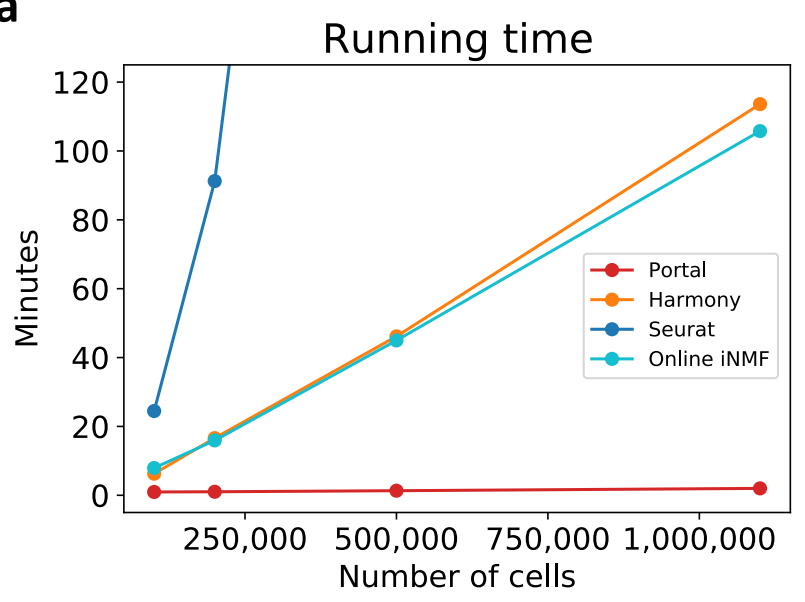

b

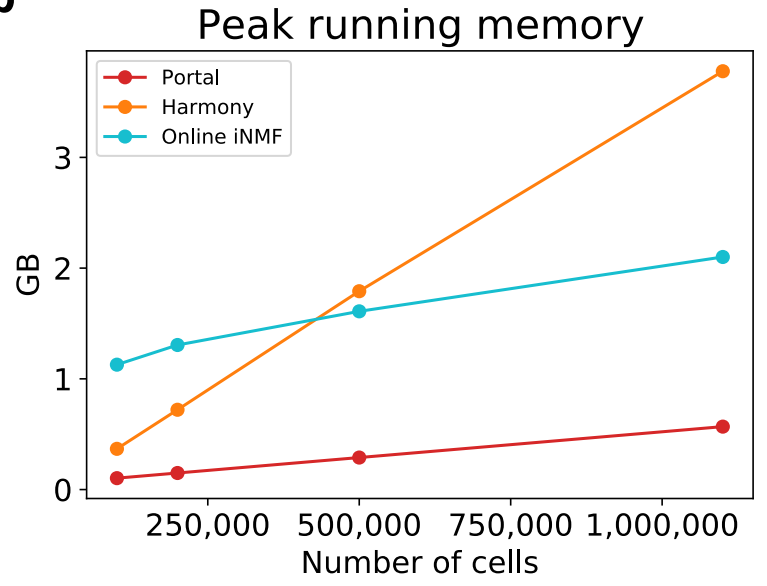

C

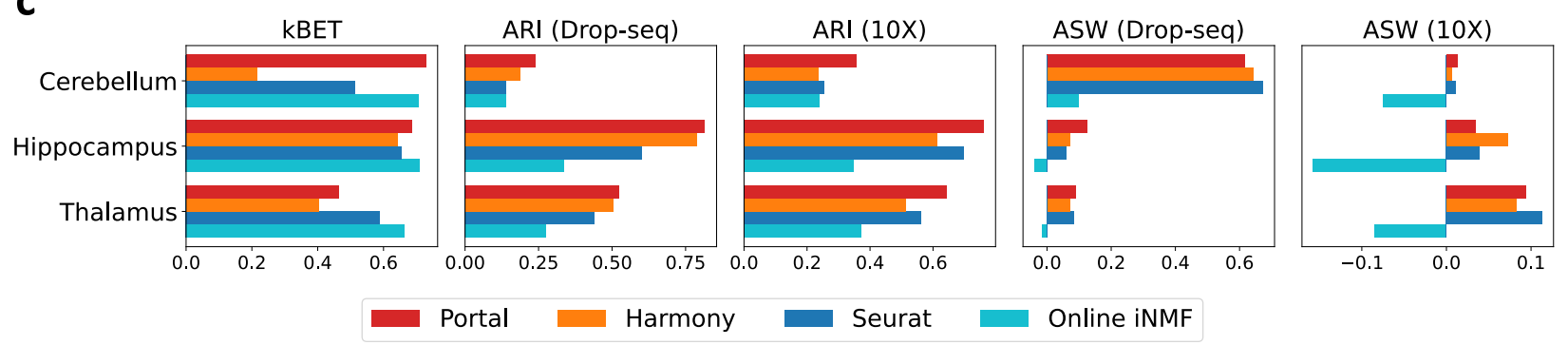

Figure 3: Benchmark of Portal, Harmony, Seurat and online iNMF. a, b. Running time and peak running memory required by benchmarked methods. The datasets were sampled from two mouse brain atlas datasets $(n=100,000,250,000,500,000$, and 1, 100, 167). Seurat requires $24.52 \mathrm{~GB}$ on the dataset with 100,000 cells, which is not comparable to the other three benchmarked methods in terms of peak running memory usage. c. Alignment (kBET) and cluster preservation performance (ARI and ASW) evaluated using three shared tissues from two mouse brain atlases (profiled by Drop-seq and 10X), including cerebellum, hippocampus, and thalamus. Cluster preservation performance was assessed based on fine-grained annotations provided by the original publications [8, 9]. A full comparison among all methods is provided in Supplementary Information (Fig. S5).

Next, we evaluated the speed, memory usage, alignment quality, and integration accuracy using a more challenging integration task. We used two mouse brain atlases [8, 9] as benchmarking datasets for a more in-depth comparison of Portal and three other methods. One atlas contains Drop-seq data of 939,489 cells, and another one contains 10X Genomics (10X) data of 160,678 cells. These two mouse brain atlases have data from three shared brain regions: cerebellum, hippocampus, and thalamus. There are many small clusters of neuron subtypes in these datasets, where gene expressions between subclusters could have a relatively small difference. Thus, these datasets are more challenging to integrate compared to data with clear clustering patterns. 
First, Portal has superior integration accuracy even when handling datasets which contain many subclusters with small difference. The ARI and ASW show that Portal outperforms other state-of-the-art methods in cluster identity preservation. In particular, for all three brain regions tested, Portal has the highest ARI score among all the benchmarked methods (Fig. 3r).

Second, Portal also outperforms the other three methods on scalability, in terms of time and memory consumption. For this benchmark test, we obtained datasets from the original full-sized datasets by combining the two atlases and subsampling proportionally from each atlas, with each dataset having increasing sample size ranging from 100,000 to 1,100,167 (full dataset). The running time and peak running memory of all methods were recorded using these datasets on the same GPU server. The results show that Portal's running time and peak running memory remained almost constant even when the sample size increased dramatically (Fig. 3a, b). Compared to the other three methods, the running time required by Portal was also substantially less (Fig. 3a). On the dataset containing 500,000 cells, Portal's running time was 80 seconds; when number of cells grew to 1,100,167, Portal's running time only increased to 120 seconds. In comparison, Harmony and online iNMF both needed more than 40 minutes to integrate 500,000 cells and more than 100 minutes to complete the integration of the full dataset. The running time of Seurat increased most rapidly among the compared methods. It took as much as 511 minutes (over 8.5 hours) to integrate the 500,000-cell dataset. The computational efficiency of Portal is owing to two important factors in its design: 1) its algorithm takes advantage of GPU-accelerated stochastic optimization, such that Portal reads data in mini-batches from the disk rather than having to load the entire dataset at once, which enables fast integration of large single-cell datasets using small amounts of memory; and 2) lightweight neural networks are adopted in Portal to further improve computational efficiency. As such, Portal is also the most memory-efficient approach among the benchmarked methods (Fig. 3b). Peak running memory required by Portal ranged from 0.10 GB on 100,000-cell dataset to $0.29 \mathrm{~GB}$ on the full million-cell dataset. Notably, Portal's lightweight networks and mini-batch stochastic optimization algorithm enable us to control GPU peak running memory usage at a constant level of 0.06 GB. Among compared methods, online iNMF used less memory than Harmony and Seurat when the sample size became larger than 500,000, because it is also trained in mini-batches. However, its peak running memory was 2.10 GB on the million-cell dataset, which is 6 times more than Portal's. Seurat required remarkably more memory usage 
than the other three methods. For clarity of visualization, we did not display the peak running memory required by Seurat as it ranged from 24.52 GB on the 100,000-cell dataset to 276.41 GB on the 500,000-cell dataset.

Finally, and importantly, Portal's high performance in speed and memory consumption does not compromise its ability to align cell type clusters. The kBET shows that Portal's alignment ability is comparable to, if not better than, the other benchmarked methods, indicating that Portal is capable to effectively remove domain-specific effects.

\section{Portal preserves subcluster and small cluster identities in complex tissues thereby facilitating identification of rare subpopulations.}

When integrating complex tissues, one problem that can arise is the inadvertent loss of small cell populations and subpopulations. Due to more nuanced differences between clusters, or due to the imbalance in cell numbers for very small cell populations, these "fine-grained" groups of cells may become inappropriately combined with other groups after integration. In the brain, for example, there are many subpopulations of neurons which are distinguished from each other using a few key gene markers while still all bearing the neuron signature; furthermore, some of these neuronal subtypes could be rare compared to other subtypes. To demonstrate that Portal can preserve the nuanced information of such small cell populations and subpopulations, we performed further analysis on the mouse hippocampus tissue integration results. Both mouse brain atlas datasets contain extensive data for this brain region (Fig. 4), and both studies identified a wide range of transcriptionally distinct cell subpopulations, including a variety of neuron subtypes, whose nuanced transcriptional differences should ideally be preserved by integration methods.

After applying Portal and the other three benchmarked methods to integrate the data, we used the integrated cell representations to perform clustering. Using the Louvain method [34] with default resolution, we obtained 29 (Portal), 29 (Harmony), 25 (Seurat) and 30 (online iNMF) clusters, respectively (Fig. S6). Particularly, we focused on one region where the cell proportions between two datasets were highly unbalanced, as marked in Fig. 4a. Only a few of cells in this region are from the 10X dataset, making it challenging to build alignment between datasets while preserving subpopulations from the Drop-seq dataset. In the original publication [8], cells from the Drop-seq dataset within the marked region were all annotated 

available under aCC-BY-NC-ND 4.0 International license.

a
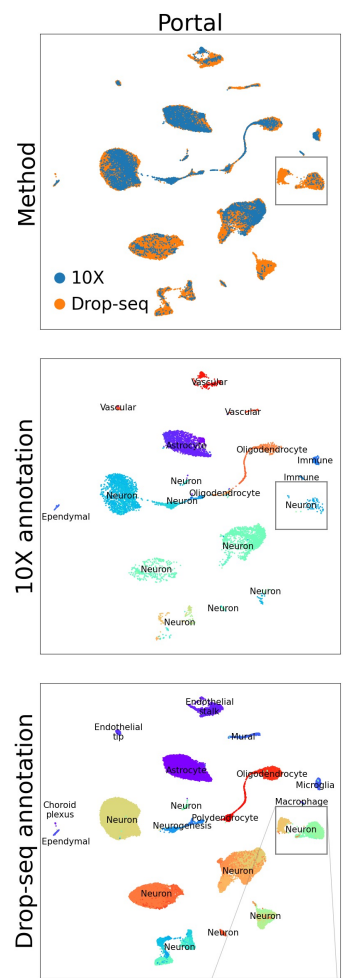

b
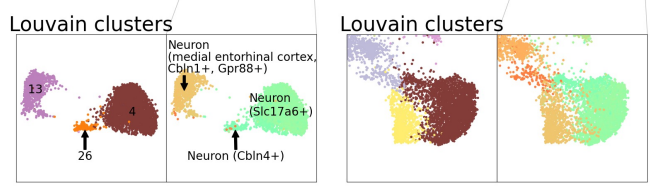

C
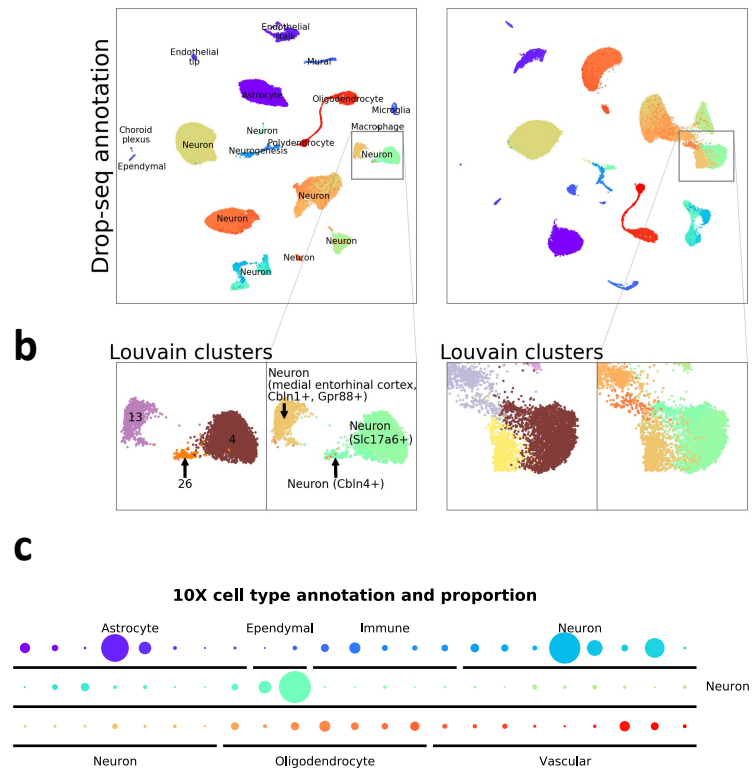
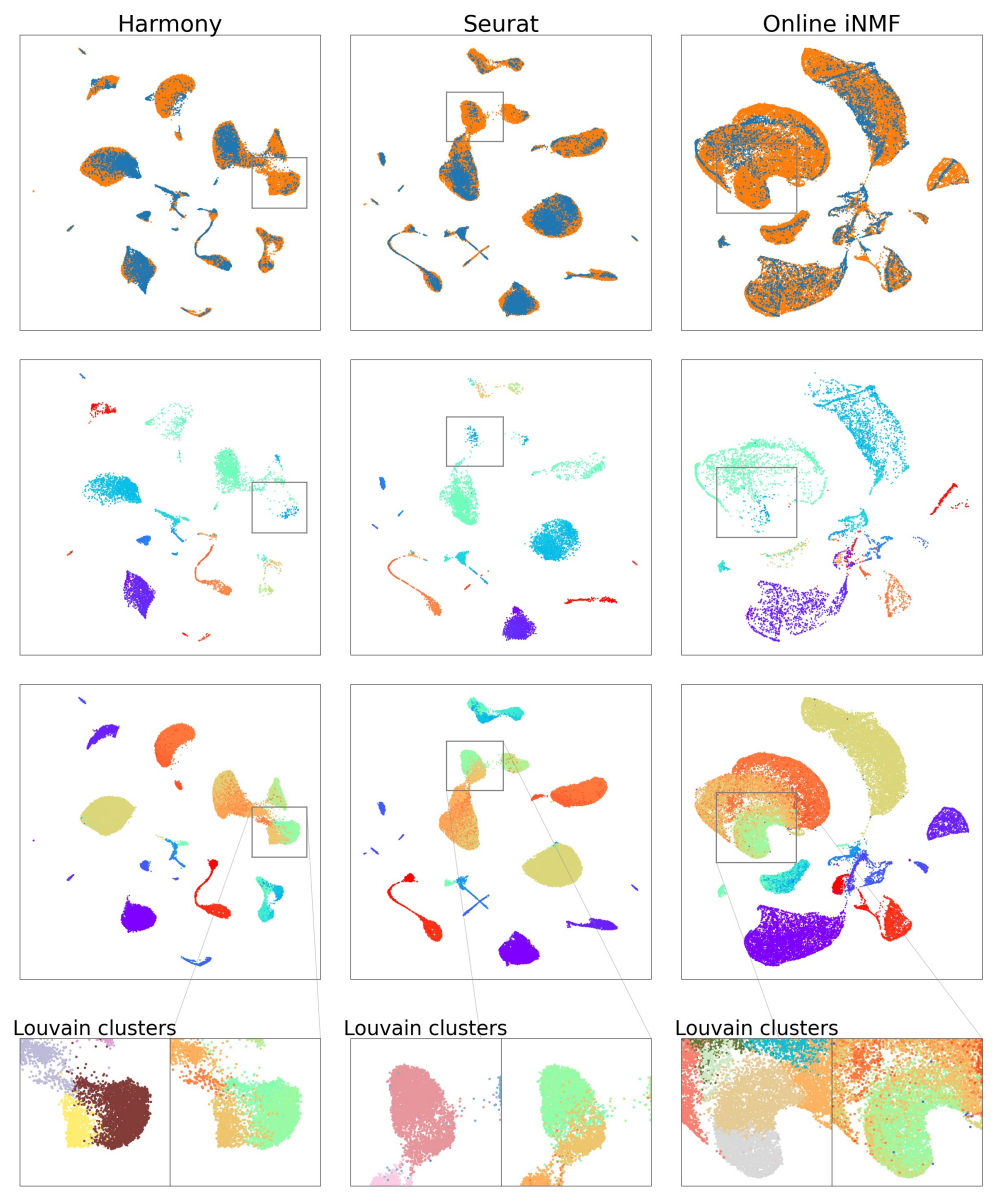

Figure 4: Preservation of fine-grained neuron subpopulations in the integration of hippocampus datasets. a. We visualized integration results from Portal, Harmony, Seurat and online iNMF of hippocampus datasets profiled by Drop-seq and 10X with UMAP. Top panels are UMAP plots colored by profiling methods. Middle and bottom panels are UMAP plots of cells from the 10X dataset, the Drop-seq dataset after integration respectively, colored by fine-grained annotations (c). b. We marked a region containing three distinct neuron subpopulations. Results from Louvain clustering algorithm were presented for a comparison of cluster identity preservation performance. c. Cell type annotations and proportions of the two datasets from their original publications [8, 9]. The comparison among proportions of subpopulations was visualized by the sizes of corresponding dots.

as neurons but further classified into three transcriptionally distinct subpopulations, namely:

Cbln1+/Grp88+ medial entorhinal cortex neurons; Slc17a6 + neurons; and Cbln4+ neurons.

Among the benchmarked methods, Portal was the only method that clearly clustered these 
cells into three coherent groups in the integrated embedding space. Specifically, clusters 4, 13, 26 identified by the Louvain method recovered the Slc17a6+ neuron; Cbln1+/Grp88+ medial entorhinal cortex neuron; and the Cbln4+ neuron subpopulations, respectively (Fig. 4b). Each cluster was confirmed by the high expression level of the annotated marker genes (Fig. S7a). Notably, these three groups only accounted for $4.79 \%, 1.76 \%$ and $0.32 \%$ of the total sample size, respectively, demonstrating Portal's ability to preserve identities of rare subpopulations. However, the differences among these three subpopulations were not well preserved by the other three methods, making it difficult to detect them each distinctly using the Louvain clustering method (Fig. 4a, b). As shown in Fig. S7k, we also identified eight protein coding genes that were the most significantly differentially expressed among clusters, indicating the different functions of each of the three neuron subtypes. Cluster 4 showed high expression levels of Camk2n1, Map1b, Nrgn, Syt1, and no detectable expression of Camk2d, Igfbp5, Nr4a2 and Ntng1. A different pattern was observed in cluster 13: High expression of Camk2d, Camk2n1, Map1b and Syt1, and no detectable expression of the other four genes. Cluster 26, meanwhile, showed moderate levels of expression of all eight genes. In the marked region, cells from the 10X dataset were mainly concentrated in cluster 4. The alignment by Portal was confirmed by the consistent gene expression levels seen in cluster 4 between the two datasets (Fig. S7b). Besides the eight differentially expressed genes, we also examined a larger set of genes, and computed the cross correlation of these genes pairwise between cells from all three groups. This analysis showed that cells within each cluster had higher similarity in gene expression than cells from other clusters, further showing the biological difference between these three clusters that should not be mixed after integration. The above results highlight Portal's power to preserve rare cell types (Fig. S7d).

The integrative analysis on the hippocampus tissue demonstrates Portal's ability to maintain nuanced transcriptional differences for small subpopulations. This means that Portal can also be used to "call out" rare subpopulations in one dataset based on integration with another dataset via label transfer. To illustrate this feature, we take 10X and SMART-seq2 (SS2) data generated for a mouse lung scRNA-seq atlas [7] as an example: the typically larger sample size of the 10X dataset facilitates powerful clustering analyses for identification of cell types; while the greater sequencing depth and sensitivity of SS2 enables deeper investigation into cell biology [35]. To leverage the different strengths of the two technologies, we used Portal to perform 
integrated analysis on 1,676 SS2 cells and 5,404 10X cells (Fig. S8a). Specifically, we defined the 10X dataset annotations from the original publication [7] as reference labels (Fig. [S8b), then made use of the Portal's integration results to identify cell types for the SS2 dataset based on these reference labels. After integration, for each SS2 cell, label transfer was performed by detecting its nearest neighbors among 10X cells. From this analysis, we identified four subpopulations of myeloid cells for the SS2 dataset, namely alveolar macrophages, dendritic cell and interstitial macrophages, classical monocytes, and non-classical monocytes (Fig. S8d). Transferred labels of these four subpopulations were validated by known marker gene expression levels [36]. For example, compared to classical monocytes, non-classical monocytes showed lower expression of Ccr2 and higher expressions of Treml4 (Fig. S9). Consistent with the gene expression pattern of alveolar macrophages in the 10X dataset, alveolar macrophages annotated by Portal in the SS2 dataset had high expression levels of marker genes Mrc1 and Siglec5. Notably, in the SS2 dataset, the alveolar macrophage subpopulation only accounted for $0.78 \%$ of total sample size, and could not be distinguished from the other SS2-profiled macrophages in the original publication [7]. Based on the original labels, alveolar macrophages were unidentified as they were labeled in a more general group named "dendritic cell, alveolar macrophage, and interstitial macrophage" (Fig. S8c). Making good use of the larger 10X dataset, Portal successfully identified extremely rare subpopulations within the SS2 dataset. We then used the mouse lemur bladder scRNA-seq datasets from Tabula Microcebus Consortium [37] as another example to demonstrate Portal's ability for discovering rare subpopulations via label transfer. In this example, mouse lemur bladder tissue was also profiled by both SS2 and 10X. When we integrated these datasets and transferred labels from the 10X dataset to the SS2 dataset using Portal, we were able to distinguish a very small myofibroblast subpopulation of just 11 cells in the SS2 dataset from the rest of the fibroblasts (Fig. S10a). We verified their myofibroblast identity based on their high expressions of known marker genes ACTA2, MYH11, TAGLN [38] (Fig. S10b).

\section{Integration of comprehensive whole-organism cell atlases.}

So far, Portal has shown impressive performance in aligning tissue-level atlases where nuanced transcriptional differences among subpopulations can be maintained after integration. We next assess Portal's capabilities under another challenging scenario: integrating two atlases 

available under aCC-BY-NC-ND 4.0 International license.

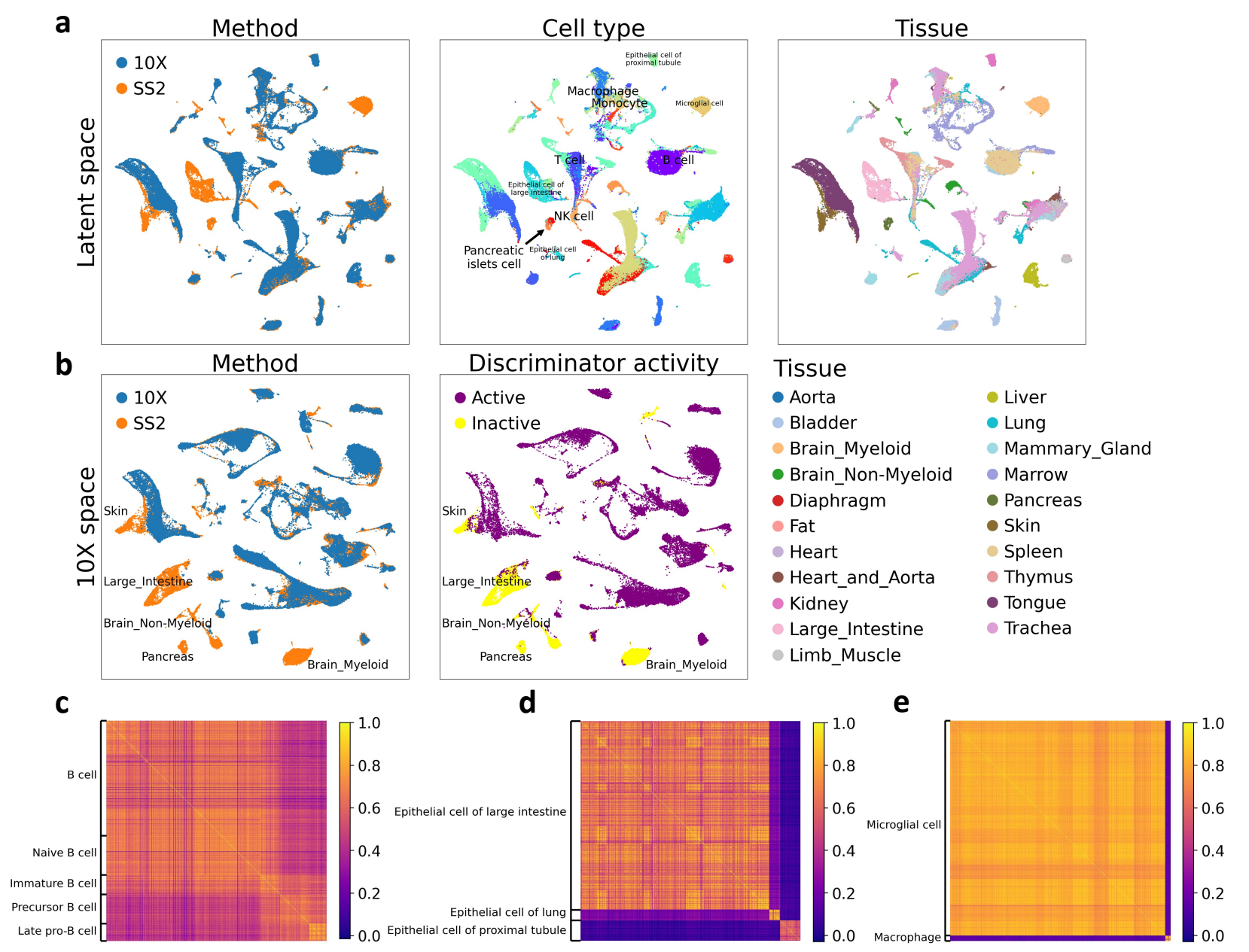

Figure 5: Construction of mouse cell atlas across entire organism by integrating atlas datasets from the Tablula Muris project. We applied Portal to integrate the datasets obtained by $10 \mathrm{X}$ and SS2. There were cells from unique tissues presented in the SS2 dataset. a. UMAP plots of Portal's integration results in the shared latent space, colored by profiling methods, cell types and tissues. b. Portal also transferred cells from the space of SS2 dataset to the space of the 10X dataset (10X space). In 10X space, 10X cells were fixed as reference. Portal only aligned SS2 cells of shared cell types between datasets to 10X cells, while maintaining the identities of SS2 cells belonging to tissue-unique cell types. This was achieved by the special design of discriminator activity in Portal. c, d. Correlations among cells from subpopulations of B cells (c) and epthelial cells (d). e. Transcriptional distinction between macrophage and microglial cells.

across an entire organism, where one of the atlases includes many more organs and tissue types than the other. This is known to be problematic for some integration algorithms due to having "missing cell types" in one of the datasets [24]. In contrast to these approaches, Portal uses discriminators with tailored design in the adversarial domain translation framework to distinguish domain-specific cell types from cell types shared across domains automatically, and 
is thus robust to non-overlapped tissue samples.

To build a foundation for extensive study of cell populations across the whole organism, Tabula Muris Consortium [7] profiled cells from 20 tissues using a combination of SS2 (44,779 cells) and 10X (54,865 cells) (Fig. 5). Notably, seven of these 20 tissues were only profiled by SS2 but not 10X: brain (myeloid and non-myeloid), diaphragm, fat, large intestine, pancreas and skin. We used Portal to build a comprehensive integrated mouse atlas that merges all the cells, and we found Portal to show extraordinary accuracy in aligning cells of the same cell type from the two datasets profiled by different platforms, not only in the shared latent space but also in both domains (Figs. 5a, b and S11). After Portal integration, tissue-specific cell types of SS2-only tissues, such as microglial cells in brain (myeloid), cell types in large intestine, and pancreatic islets cells, were all successfully and correctly remained separated from other cell types. The other three benchmarked methods, however, failed to retain many tissue-specific cell types unmixed with other cell types. For instance, they mixed microglial cells together with other macrophage cells, even though the data from these two cell types were clearly transcriptionally different (Figs. 5e, S11).

Using this construction of a mouse cell atlas across organs, we also confirmed that the designed boundaries for discriminator active region in Portal (Fig. 1 $k$ ) indeed helped to maintain the biological variation. By looking into the domain of $10 \mathrm{X}$ data (10X space), the discriminator in the 10X domain was found inactive for tissue-specific cell types that were only in the SS2 dataset (Fig. 5b). For these cells, Portal ensured that their identities were preserved by making the adversarial learning objective inactive on them automatically. Portal's ability to conserve information of cell populations indicates its reliability for integrating atlas-level single-cell datasets across entire organisms.

Besides the alignment between datasets, Portal's integration result could characterize the similarities and differences among cell types. For example, immune cells such as B cells, T cells, natural killer cells (NK cells), monocytes and macrophages were profiled by both platforms and contained in multiple tissues including brain (myeloid), diaphragm, fat, kidney, limb muscle, liver, lung, mammary gland, marrow, spleen, and thymus. Portal correctly kept the subpopulations belonging to the same type of immune cells close to each other, revealing the resemblance of immune cells across different tissues. For instance, the transcriptional correlation of all types of B cells, containing B cells, naive B cells, immature B cells, precursor B cells, and 
late pro-B cells confirmed such similarity (Fig. 5r). In addition, the epithelial cells of different tissues were identified by Portal as disjoint clusters, which was consistent with the biological distinction among these cell types (Fig. 5d).

\section{Portal successfully and efficiently aligns single-cell RNA-seq data and single-nucleus RNA-seq data.}

For frozen samples such as biobanked tissues, and for tissue types that have unique morphology or phenotypes, such as brain, fat, or bone, it can be challenging or sometimes even impossible to extract intact cells for scRNA-seq profiling [39, 40]. To bypass this issue, single-nucleus RNA sequencing (snRNA-seq) has been developed. Although nuclear transcriptomes are shown to be representative of the whole cell [41], distinctions between the whole cell and nucleus in terms of the transcript type and composition make scRNA-seq data and snRNA-seq data intrinsically different [39]. Aligning these two types of data is desirable, as the combined dataset enables joint analysis that can take advantages of both techniques, and help to improve statistical power for the analysis. Especially for comparing multiple complex tissues, with some cell types being shared and others being non-overlapping, researchers could benefit from such integrated joint analysis - one example being the integration of brain snRNA-seq data with scRNA-seq data of blood to examine similarities and differences between immune cells in each tissue milieu. However, due to the inherent difference in these two data types, aligning scRNA-seq and snRNA-seq data is not the same as batch effects correction. Compared to batch effects among scRNA-seq datasets, technical noise and unwanted variation arising from different data types are often more complex and have higher strength [39, 42]. Thus, using standard batch effects correction to integrate across data types may result in loss of alignment accuracy or important biological signals.

We evaluated Portal's ability to integrate snRNA-seq data and scRNA-seq data using three mouse brain atlas datasets, including one snRNA-seq dataset profiled by SPLiT-seq [43, and two scRNA-seq datasets profiled by Drop-seq and 10X [8, 9]. In this task, we applied integration methods to harmonize these three atlases across all brain regions. To test the accuracy of integration results, we only used cells that had annotations provided by the authors in each atlas project. After selecting cells with cell type annotations, 319,359 cells in the Drop-seq dataset, 160,678 cells in the 10X dataset, and 74,159 nuclei in the SPLiT-seq remained for 
integration.

Prior to any integration, the raw datasets were clustered by the experimental method rather than the cell type (Fig. S12a), and shared cell types between the three datasets did not align well, indicating the initial discrepancy between the three large datasets. After integration, UMAP visualizations showed that the different alignment methods gave varying results. Portal (Fig. S12b) and Seurat (Fig. S12d) achieved the best alignment of data across different methods, showing good mixing of cells annotated with the same cell type label, while also preserving subcluster data structure in the integrated results. In particular, the alignment of scRNA-seq (10X, Drop-seq) and snRNA-seq (SPLiT-seq) datasets was comparably good as that of the two scRNA-seq datasets, indicating successful alignment between the two data types without loss of biologically important variations between clusters. Online iNMF (Fig. S12 e), although it successfully clustered and aligned the same cell types together, within each cluster the streaky pattern suggested potential numerical artefacts in the integrated data. Furthermore, online iNMF alignment resulted in loss of biological variation, which was most easily observable in the coalescence of the previously distinct neuron subpopulations (Fig. S12a) into one large amorphous cluster (Fig. S12e). Harmony, however, showed poor mixing of the snRNA-seq data in some of the cell types, such as the astrocytes, where the scRNA-seq datasets were well-mixed after alignment, but the snRNA-seq data were not mixed well with the rest (Fig. S12 c). Similar to online iNMF, some of the neurons' subcluster structure appeared to be lost after the integration by Harmony. Overall, Portal and Seurat presented the best scRNA-seq and snRNA-seq data alignment performance; however, not including data preprocessing time, Seurat took over 17 hours to complete the task, while Portal only took 87 seconds.

\section{Portal aligns spermatogenesis differentiation process across multiple species.}

Portal does not need to specify the structure and the strength of unwanted variation when integrating datasets. Instead, it can flexibly account for general difference between datasets, including batch effects, technical noises, and other sources of unwanted variation, by nonlinear encoders and generators in the adversarial domain translation framework. Therefore, Portal is also applicable for merging datasets with intrinsic biological divergence, revealing biologically meaningful connections among these datasets. In this section, we demonstrate that Portal 
bioRxiv preprint doi: https://doi.org/10.1101/2021.11.16.468892; this version posted November 19,2021 . The copyright holder for this preprint (which was not certified by peer review) is the author/funder, who has granted bioRxiv a license to display the preprint in perpetuity. It is made available under aCC-BY-NC-ND 4.0 International license.

a

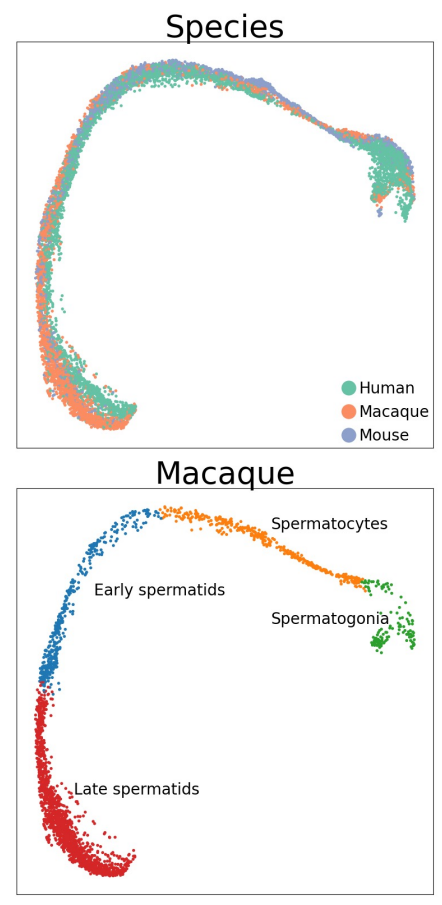

C

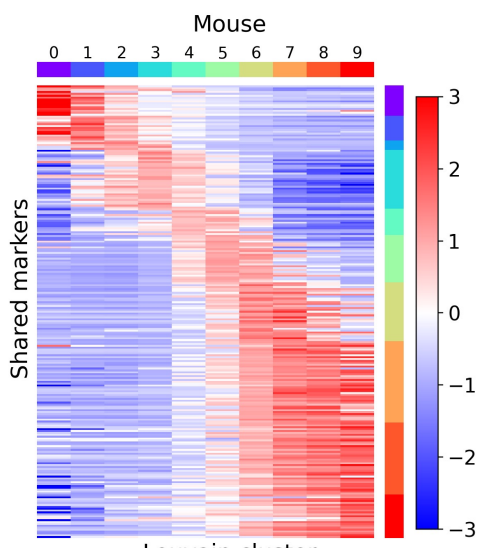

Louvain cluster

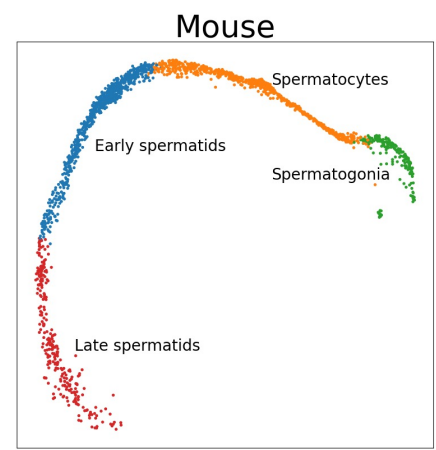

Human

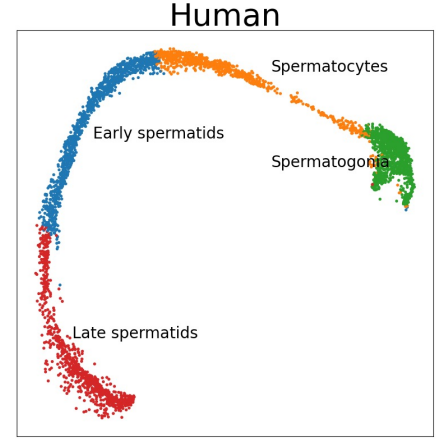

b

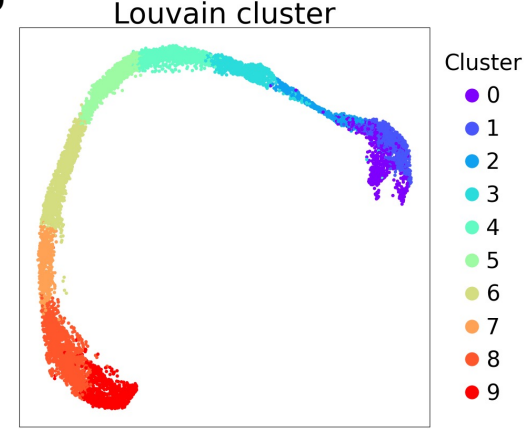

Figure 6: Integration of spermatogenesis datasets across different species, including mouse, macaque and human. a. UMAP plot of Portal's result colored by species, as well as UMAP plots of integrated mouse, macaque, human datasets visualized separately. b. Ten clusters were obtained by applying the Louvain clustering algorithm, facilitating detailed comparative analysis across species. c. Portal identified 239 highly variable genes that are shared in the spermatogenesis process across all three mammalian species.

can successfully align scRNA-seq datasets of the testes from different species including mouse, macaque and human (Fig. 6).

Compared to merging datasets from the same species, cross-species integration poses additional unique challenges. Although the transcriptomes of different species may share expression of homologous or orthologous genes, the number of shared genes varies between 
different species and is limited. Furthermore, two species may have genes with very similar sequence and be annotated in the transcriptome by the same name, but have altered function, which means that expression of the same gene in different species can denote different cell function [44]. In other words, the amount of information one can utilize for integration becomes limited and fuzzier while the variation across datasets becomes far larger, with limited number of shared genes and even fewer shared highly variable genes across different species. Nonetheless, cross-species integration can be very meaningful despite its challenges, as it can generate quick draft annotations of new or less-studied species' atlases and cell types via label transfer from well-studied species. This saves time in the manual annotation process of single-cell tissue atlas generation for new species. Such integration can also enable detailed comparisons between species, such as comparisons of cell type composition, discovery of cell types unique to a particular species, or cross-species comparisons of the same cell types.

Mammalian spermatogenesis is a continuous and irreversible differentiation process from spermatogonial stem cells (SSCs) to sperm cells [45, 46, 47, 48, 16]. Due to the unique degenerate nature of the $\mathrm{Y}$ chromosome (Y-chr), Y-chr gene expression is intricately and tightly regulated in the spermatogenesis process through meiotic sex chromosome inactivation (MSCI) 49, 50, 51, 52, 53. Interestingly, Y-linked genes are highly divergent between different species, including between closely related primates such as the chimpanzee, macaque, and human [49, 54, 55]; yet MSCI as a process is conserved across many species and is required for male fertility [52, 56]. This evidence suggests that while the evolution of genes on the Y-chr generated diverse species-specific genetic combinations, the tight control of gene expression through MSCI is required to ensure genetic stability [49]. Recently, cross-species comparisons of "escape genes" that are able to maintain or re-activate their expression despite MSCI repression during spermatogenesis have generated fascinating insights on evolutionary biology, and on sex chromosome evolution [51, 53, 57, 16]. In this biological context, integrating datasets with continuous and gradient developmental trajectories, such as for spermatogenesis data, requires integration methods to preserve the continuous structure of each dataset, while still providing high accuracy of cell type alignment between datasets. This is more difficult when, like in spermatogenesis data, there are no distinct clusters, making integration of such data a particularly difficult task. Here, we perform cross-species integration of testes datasets from three species, including one mouse [47], one macaque and one human [16], aligning the different 
stages of spermatogenesis across species thereby highlighting unique features of each. The successful integration of these spermatogenesis trajectories serves as a demonstration of the power of Portal in complex and low-information data alignment, and how it can facilitate the annotation and discovery process for new single-cell tissue atlases.

We first annotated the mouse sample according to the pattern of marker genes (Spermatogonia: Sycp1, Uchl1, Crabp1, Stra8; Spermatocytes: Piwil1, Pttg1, Insl6, Spag6; Early spermatids: Tssk1, Acrv1, Spaca1, Tsga8; Late spermatids: Prm1, Prm2, Tnp1, Tnp2) [45, 46]. Then we used Portal to harmonize the three samples, where the integration was accomplished in the mouse sample domain: The cells from the mouse sample were used as reference, and cells from the other two species were mapped to the mouse sample domain by Portal. Based on our annotation of the mouse sample, we transferred the broad cell type labels to cells from the macaque and human samples according to the nearest neighbors, using the alignment given by Portal (Fig. 6a). To check whether the alignments were correct for broad cell type identities, we visualized the UMAPs for cells from each species labeled by their original published annotations [16], and we confirmed concordant cell type integration across species (Fig. S13). Then, we used Louvain clustering algorithm to cluster the cells from all three species based on integrated cell representations. Ten clusters were found, and the cluster names were relabeled by their order of progression from the spermatogonia along the developmental trajectory (Fig. 6b). We then visualized the expression of known spermatogenesis markers [45, 46, 16] in each Louvain cluster and found that the Louvain clusters generated by Portal's alignment clearly captured the key transcriptomic features for each stage of spermatogenesis, and correctly identified cells from each stage for all three species (Fig. S14, S15). Furthermore, each Louvain cluster represented a more fine-grained classification of cells within the labeled broad spermatogenesis cell types. Using these clusters we assessed the transcriptomic changes throughout the differentiation trajectory with higher resolution (Fig. S14, S15). Notably, many of the marker genes known to define stages of spermatogenesis in human were not shared or sometimes not expressed in macaque and/or mouse scRNA-seq data. For example, human genes SYCP3, YBX2, SPACA4, H1FNT, PRM1, and TNP1 were known to mark human spermatogenesis progression, but they were absent in the macaque dataset. As only highly variable genes that were expressed in all three species were considered in the integration process, these genes were not used by Portal. However, they showed clear expression in the cell clusters where they were expected to be 
expressed after integration (Fig. S15), confirming the correctness of Portal's integration result.

The above results show that Portal can provide an accurate integration even for genes not measured by all three samples.

Cross-species data integration can be a quick and easy way to generate draft cell atlas annotations for new species via label transfer from well-annotated species, but moreover, such integrated data can be used to highlight interesting biological features of shared cell types. In our Louvain clusters for spermatogenesis, for each species, we selected top 200 highly expressed genes of every cluster. By taking the intersection of those genes across three species, we then identified 239 highly variable genes that are shared in the spermatogenesis process across all three mammalian species (Fig. 6c). For the highly expressed genes that were unique to only one species, we compared their expressions across all three species (Fig. S16). Such comparisons could give insight into shared and divergent features of spermatogenesis across different species.

\section{Discussion}

Taking advantage of machine learning methodologies, Portal is an efficient and powerful tool for single-cell data integration that easily scales to handle large datasets with sample sizes in the millions. As a machine learning-based model, Portal is easy to train, and its training process is greatly accelerated by using GPUs. Meanwhile, mini-batch optimization allows Portal to be trained with a low memory usage. Besides, it also makes Portal applicable in the situation where the dataset is not fully observed, but arrives incrementally.

The nonlinearity of neural networks makes Portal a flexible approach that can adjust for complex dataset-specific effects. Nonetheless, according to benchmarking studies, strong ability for removing dataset-specific effects often comes with the weakness in conserving biological variation [42, 58], e.g., being prone to overcorrection. Portal overcomes this challenge by its model and algorithm designs. First, the boundaries of discriminator scores help Portal to protect dataset-unique cell types from overcorrection. Second, the use of three specifically designed regularizers not only assists Portal to find correct correspondence across domains, but also enables Portal to have high-level preservation of subcluster and small cluster identities in both datasets.

Two existing popular methods are Seurat and BBKNN. Seurat often provides integration results with high accuracy, but also requires high computational cost, preventing its usage on 
large-scale datasets; while BBKNN is well-known for its extremely fast speed, its comparatively less precise results are sometimes a concern for users (Figs. 2, S3, S4, and S5). A major advance of Portal over these existing state-of-the-art integration approaches is its ability to achieve high efficiency and accuracy simultaneously. With speed comparable or faster than BBKNN, and significantly lower memory requirement than BBKNN (Fig. S5a), Portal presents similar alignment performance as well as superior information preservation performance compared to that of Seurat (Figs. 2, 3a, $4 \mathbf{b}, \mathrm{S} 3$ and $\mathrm{S} 4$ ).

By leveraging the adversarial domain translation framework, Portal can build meaningful alignment between datasets with efficient utilization of information. From single tissue types to complex cell atlases, Portal showed extraordinary information preservation performance throughout all integration tasks. This feature of Portal is exemplified by integration of the spermatogenesis trajectory across three species, where only a limited number of highly variable genes were shared and utilized by Portal. Improvements can further be made if an effective way of leveraging the whole transcriptome of all species is developed, which is left for future work to address. Nonetheless, such cross-species integration allows biologists to easily identify shared and divergent cellular programs across different species, which is particularly useful for addressing questions of evolutionary biology. In our example of mouse, macaque, and human testes tissue integration, identifying genes that are primate-specific can help to generate hypotheses about the evolution of primates and shed light on the applicability of various animal models for biological research.

Recently, two other generative adversarial networks based approaches have been proposed for single-cell data integration, namely cross-modal autoencoders [59] and iMAP [60]. Cross-modal autoencoders rely on cell type label information or paired data to obtain accurate integration results, and such paired information may not always be available. iMAP software faces scaling challenges when working on datasets of 500,000 cells or more, due to its high GPU memory consumption; further, as a two-stage integration method, iMAP results often rely on the MNN pairs detected in the first stage as anchors. By these reasons, we believe that Portal has made significant progress in the development of single-cell methods, as it is a unified framework which utilizes advanced techniques in domain translation with its tailored designs to achieve efficiency, scalability, flexibility and accuracy simultaneously.

It is now clear that using single-cell technologies to assemble comprehensive whole organism 
atlases encompassing diverse cell types is accelerating biological discovery, and this demand will only grow as more datasets are generated. The demand for integration of such datasets, along with the size of these datasets, will expand correspondingly. We expect that Portal, with its fast, versatile, and robust integration performance, will play a valuable and essential role in the modern life scientist's single-cell analysis toolkit.

\section{Methods}

\section{The model of Portal}

Expression measurements of cells from two different studies are viewed as datasets originated from two different domains $\mathcal{X}$ and $\mathcal{Y}$. After standard data preprocessing of the expression data, Portal performs joint principle component analysis (PCA) across datasets and adopts the first $p$ principal components of cells as the low-dimensional representation of cells, namely, cell embeddings. Portal takes the cell embeddings as the input to achieve data alignment between $\mathcal{X}$ and $\mathcal{Y}$. To learn a harmonized representation of cells, Portal introduces a $q$-dimensional latent space $\mathcal{Z}$ to connect $\mathcal{X}$ and $\mathcal{Y}$, where the latent codes of cells in $\mathcal{Z}$ are not affected by domain-specific effects but capture biological variation.

Portal achieves the integration of datasets through training a unified framework of adversarial domain translation. Let $\mathbf{x}$ and $\mathbf{y}$ be the cell embeddings in $\mathcal{X}$ and $\mathcal{Y}$, respectively. For domain $\mathcal{X}$, Portal first employs encoder $E_{1}(\cdot): \mathcal{X} \rightarrow \mathcal{Z}$ to get a latent code $E_{1}(\mathbf{x}) \in \mathcal{Z}$ for all $\mathbf{x} \in \mathcal{X}$. Encoder $E_{1}(\cdot)$ is designed to remove domain-specific effects in $\mathcal{X}$. To transfer cells from $\mathcal{X}$ to $\mathcal{Y}$, Portal then uses generator $G_{2}(\cdot): \mathcal{Z} \rightarrow \mathcal{Y}$ to model the data generating process in domain $\mathcal{Y}$, where domain-specific effects in $\mathcal{Y}$ are induced. $E_{1}(\cdot)$ and $G_{2}(\cdot)$ together form a domain translation network $G_{2}\left(E_{1}(\cdot)\right)$ that maps cells from $\mathcal{X}$ to $\mathcal{Y}$ along $\mathcal{X} \rightarrow \mathcal{Z} \rightarrow \mathcal{Y}$. By symmetry, encoder $E_{2}(\cdot): \mathcal{Y} \rightarrow \mathcal{Z}$ and generator $G_{1}(\cdot): \mathcal{Z} \rightarrow \mathcal{X}$ are utilized to transfer cells from $\mathcal{Y}$ to $\mathcal{X}$ along the path $\mathcal{Y} \rightarrow \mathcal{Z} \rightarrow \mathcal{X}$.

Portal trains domain translation network $G_{2}\left(E_{1}(\cdot)\right): \mathcal{X} \rightarrow \mathcal{Y}$, such that the distribution of transferred cells $G_{2}\left(E_{1}(\mathbf{x})\right)$ can be mixed with the distribution of cell embeddings $\mathbf{y}$ in domain $\mathcal{Y}$. Discriminator $D_{2}(\cdot)$ is employed in domain $\mathcal{Y}$ to identify where the poor mixing of the two distributions occurs. The competition between domain translation network $G_{2}\left(E_{1}(\cdot)\right)$ and discriminator $D_{2}(\cdot)$ is known as adversarial learning [27]. Discriminator $D_{2}(\cdot)$ will send a 
feedback signal to improve the domain translation network $G_{2}\left(E_{1}(\cdot)\right)$ until the two distributions are well mixed. By symmetry, domain translation network $G_{1}\left(E_{2}(\cdot)\right): \mathcal{Y} \rightarrow \mathcal{X}$ and discriminator $D_{1}(\cdot)$ deployed in domain $\mathcal{X}$ form another adversarial learning pair. The feedback signal from $D_{1}(\cdot)$ improves $G_{1}\left(E_{2}(\cdot)\right)$ until the well mixing of the transferred cell distribution $G_{1}\left(E_{2}(\mathbf{y})\right)$ and the original cell distribution $\mathrm{x}$ in domain $\mathcal{X}$.

Notice that the well mixing of the transferred distribution and the original distribution does not necessarily imply the correct correspondence established between $\mathcal{X}$ and $\mathcal{Y}$. First, cells from a unique cell population in domain $\mathcal{X}$ should not be forced to mix with cells in domain $\mathcal{Y}$. Second, cell types $A$ and $B$ in domain $\mathcal{X}$ could be incorrectly aligned with cell types $B$ and $A$ in domain $\mathcal{Y}$, respectively, even if the two distributions are well mixed. These problems can occur because we don't have any cell type label information as an anchor for data alignment across domains. To address these, Portal has the following unique features, distinguishing it from existing domain translation methods [28, 29]. First, Portal has a tailored discriminator for the integrative analysis of single-cell data, which can prevent mixing of unique cell types in one domain with a different type of cell in another domain. Second, Portal deploys three regularizers to find correct correspondence during adversarial learning; These regularizers also play a critical role in accounting for domain-specific effects and retaining biological variation in the shared latent space $\mathcal{Z}$.

We propose to train domain translation networks under the following framework:

$$
\begin{aligned}
\min _{\left\{E_{1}, G_{1}, E_{2}, G_{2}\right\}} \max _{\left\{D_{1}, D_{2}\right\}} & \mathcal{L}_{\mathcal{X}}\left(D_{1}, E_{2}, G_{1}\right)+\mathcal{L}_{\mathcal{Y}}\left(D_{2}, E_{1}, G_{2}\right), \\
\text { subject to } & \mathcal{R}_{\mathrm{AE}}\left(E_{1}, G_{1}, E_{2}, G_{2}\right) \leq t_{\mathrm{AE}} \\
& \mathcal{R}_{\mathrm{LA}}\left(E_{1}, G_{1}, E_{2}, G_{2}\right) \leq t_{\mathrm{LA}} \\
& \mathcal{R}_{\mathrm{cos}}\left(E_{1}, G_{1}, E_{2}, G_{2}\right) \leq t_{\mathrm{cos}}
\end{aligned}
$$

where component (2) is the objective function of adversarial learning for single-cell data integration; components (3), (4) and (5) are regularizers for imposing the autoencoder consistency, the latent alignment consistency and cosine similarity to preserve cross-domain correspondence, respectively. We have investigated the roles of each component in Portal and provided more results (Figs. S1 and S2 in the Supplementary Information. We explain each component in more detail in the next section.

Adversarial learning with discriminator score thresholding. The adversarial training between discriminators and domain translation networks is formulated as a min-max opti- 

available under aCC-BY-NC-ND 4.0 International license.

mization problem (2), where $\mathcal{L}_{\mathcal{X}}\left(D_{1}, E_{2}, G_{1}\right)=\mathbb{E}\left[\log D_{1}(\mathbf{x})\right]+\mathbb{E}\left[\log \left(1-D_{1}\left(G_{1}\left(E_{2}(\mathbf{y})\right)\right)\right)\right]$ and $\mathcal{L}_{\mathcal{Y}}\left(D_{2}, E_{1}, G_{2}\right)=\mathbb{E}\left[\log D_{2}(\mathbf{y})\right]+\mathbb{E}\left[\log \left(1-D_{2}\left(G_{2}\left(E_{1}(\mathbf{x})\right)\right)\right)\right]$ are the objective functions for adversarial learning in domain $\mathcal{X}$ and domain $\mathcal{Y}$, respectively. Given domain translation network $G_{1}\left(E_{2}(\cdot)\right)$, discriminator $D_{1}(\cdot): \mathcal{X} \rightarrow(0,1)$ is trained to distinguish the transferred cells $G_{1}\left(E_{2}(\mathbf{y})\right)$ from the original cells $\mathbf{x}$, where a high score (close to 1 ) indicates a "real cell" in domain $\mathcal{X}$, and a low score (close to 0) indicates a "transferred cell" from domain $\mathcal{Y}$. This is achieved by maximizing $\mathcal{L}_{\mathcal{X}}$ with respect to $D_{1}(\cdot)$. Similarly, discriminator $D_{2}(\cdot)$ in domain $\mathcal{Y}$ is updated by maximizing $\mathcal{L}_{\mathcal{Y}}$. Given discriminators $D_{1}(\cdot)$ and $D_{2}(\cdot)$, the domain translation networks are trained by minimizing $\mathcal{L}_{\mathcal{X}}+\mathcal{L}_{\mathcal{Y}}$ with respect to $E_{1}(\cdot), G_{2}(\cdot)$ and $E_{2}(\cdot), G_{1}(\cdot)$, such that the discriminators cannot distinguish transferred cells from real cells. This is equivalent to $\min _{\left\{E_{1}, G_{1}, E_{2}, G_{2}\right\}} \mathbb{E}\left[\log \left(1-D_{1}\left(G_{1}\left(E_{2}(\mathbf{y})\right)\right)\right)\right]+\mathbb{E}\left[\log \left(1-D_{2}\left(G_{2}\left(E_{1}(\mathbf{x})\right)\right)\right)\right]$. However, direct optimization of this objective function is known to suffer from severe gradient vanishing [27, 61]. Therefore, we adopt the "logD-trick" [27] to stabilize the training process. Denote $\mathcal{L}_{\mathcal{X}}^{\log D}=-\mathbb{E}\left[\log D_{1}\left(G_{1}\left(E_{2}(\mathbf{y})\right)\right)\right]$ and $\mathcal{L}_{\mathcal{Y}}^{\log D}=-\mathbb{E}\left[\log D_{2}\left(G_{2}\left(E_{1}(\mathbf{x})\right)\right)\right]$. In practice, we $\operatorname{minimize} \mathcal{L}_{\mathcal{X}}^{\log D}+\mathcal{L}_{\mathcal{Y}}^{\log D}=-\left\{\mathbb{E}\left[\log D_{1}\left(G_{1}\left(E_{2}(\mathbf{y})\right)\right)\right]+\mathbb{E}\left[\log D_{2}\left(G_{2}\left(E_{1}(\mathbf{x})\right)\right)\right]\right\}$ with respect to $E_{1}(\cdot), G_{2}(\cdot)$ and $E_{2}(\cdot), G_{1}(\cdot)$, instead of minimizing $\mathcal{L}_{\mathcal{X}}+\mathcal{L}_{\mathcal{Y}}=\mathbb{E}\left[\log \left(1-D_{1}\left(G_{1}\left(E_{2}(\mathbf{y})\right)\right)\right)\right]+$ $\mathbb{E}\left[\log \left(1-D_{2}\left(G_{2}\left(E_{1}(\mathbf{x})\right)\right)\right)\right]$.

Although the above adversarial learning can make the transferred cells and real cells well mixed, it can falsely force cells of a unique cell population in one domain to mix with cells in another domain, leading to overcorrection. Consider a cell population that is present in $\mathcal{X}$ but absent in $\mathcal{Y}$ as an example. On one hand, discriminator $D_{1}(\cdot)$ can easily identify cells from the unique cell population as real cells in $\mathcal{X}$. Cells in the nearby region of this cell population have extremely high discriminator scores. Some cells in $\mathcal{Y}$ will be mapped into this region by the domain translation network $G_{1}\left(E_{2}(\cdot)\right)$, leading to incorrect mixing of cell types in $\mathcal{X}$. On the other hand, cells transferred from $\mathcal{X}$-unique population will have low $D_{2}$ scores in $\mathcal{Y}$. Discriminator $D_{2}(\cdot)$ will incorrectly force the domain translation network $G_{2}\left(E_{1}(\cdot)\right)$ to mix these cells with real cells in domain $\mathcal{Y}$. The cell identity as a domain-unique population in $\mathcal{X}$ is lost.

From the above reasoning, domain-unique cell populations are prone to be assigned with extreme discriminator scores, either too high in the original domain or too low in the transferred domain. Such extreme scores can lead to overcorrection. To address this issue in single-cell data 
integration tasks, we set boundaries for discriminator scores to make discriminators inactive on such cells. Specifically, the outputs of standard discriminators are transformed into $(0,1)$ with the sigmoid function, i.e., $D_{i}(\mathbf{x})=\operatorname{sigmoid}\left(d_{i}(\mathbf{x})\right)=1 /\left(1+\exp \left(-d_{i}(\mathbf{x})\right)\right), i=1,2$, where $d_{i}(\mathbf{x}) \in(-\infty, \infty)$ is the logit of the output. We bound the discriminator score by thresholding its logit to a reasonable range $[-t, t]$ :

$$
\widetilde{D}_{i}(\mathbf{x})=1 /\left(1+\exp \left(-\operatorname{clamp}\left(d_{i}(\mathbf{x})\right)\right)\right)
$$

where $\operatorname{clamp}(\cdot)=\max (\min (\cdot, t),-t)$. By clamping the logit $d_{i}(\mathbf{x}), \widetilde{D}_{i}(\mathbf{x})$ becomes a constant when $d_{i}(\mathbf{x})<-t$ or $d_{i}(\mathbf{x})>t$, providing zero gradients for updating the parameters of encoders and generators. Meanwhile, $\widetilde{D}_{i}(\mathbf{x})$ remains the same as $D_{i}(\mathbf{x})$ when $d_{i}(\mathbf{x}) \in[-t, t]$. By such design, the adversarial learning mechanism in Portal is only applied to cell populations that are likely to be common across domains. In Portal, we then use this modified version of discriminators $\widetilde{D}_{i}(\cdot)$ to avoid incorrect alignment of domain-unique cell populations. For clarity, we still use the notation $D_{i}(\cdot)$ to represent $\widetilde{D}_{i}(\cdot)$ hereinafter.

Regularization for autoencoder consistency. Encoder $E_{1}(\cdot): \mathcal{X} \rightarrow \mathcal{Z}$ and generator $G_{1}(\cdot): \mathcal{Z} \rightarrow \mathcal{X}$ form an autoencoder structure, where $E_{1}(\cdot)$ removes domain-specific effects in $\mathcal{X}$, and $G_{1}(\cdot)$ recovers them. Similarly, $E_{2}(\cdot): \mathcal{Y} \rightarrow \mathcal{Z}$ and $G_{2}(\cdot): \mathcal{Z} \rightarrow \mathcal{Y}$ form another autoencoder structure. Therefore, we use the regularizer in (3) for the autoencoder consistency, where $\mathcal{R}_{\mathrm{AE}}=\frac{1}{p}\left\{\mathbb{E}\left[\left\|\mathbf{x}-G_{1}\left(E_{1}(\mathbf{x})\right)\right\|_{2}^{2}\right]+\mathbb{E}\left[\left\|\mathbf{y}-G_{2}\left(E_{2}(\mathbf{y})\right)\right\|_{2}^{2}\right]\right\}, p$ is the dimensionality of $\mathcal{X}$ and $\mathcal{Y}$.

Regularization for cosine similarity correspondence. Besides the autoencoder consistency, the cosine similarity regularizer in (5) plays a critical role in data alignment between domains, where $\mathcal{R}_{\cos }=\mathbb{E}\left[1-\frac{<\mathbf{x}, G_{2}\left(E_{1}(\mathbf{x})\right)>}{\|\mathbf{x}\|_{2}\left\|G_{2}\left(E_{1}(\mathbf{x})\right)\right\|_{2}}\right]+\mathbb{E}\left[1-\frac{<\mathbf{y}, G_{1}\left(E_{2}(\mathbf{y})\right)>}{\|\mathbf{y}\|_{2}\left\|G_{1}\left(E_{2}(\mathbf{y})\right)\right\|_{2}}\right]$ is the regularizer that imposes the cross-domain correspondence on domain translation. The key idea is that a cell and its transferred version should not be largely different from each other in terms of cosine similarity. This is because cosine similarity is scale invariant and insensitive to domain-specific effects, including differences in sequencing depth and capture efficiency of protocols used across datasets [62, 24, 21]. Thus, the cosine similarity regularizer is helpful to uncover robust correspondence between cells of the same cell type across domains.

\section{Domain-specific effects removal in the shared latent space by latent alignment regu-}

larization. Portal decouples domain translation into the encoding process $\mathcal{X} \rightarrow \mathcal{Z}$ (or $\mathcal{Y} \rightarrow \mathcal{Z}$ ) and the generating process $\mathcal{Z} \rightarrow \mathcal{Y}$ (or $\mathcal{Z} \rightarrow \mathcal{X}$ ). Although adversarial learning enables the do- 
main translation networks to effectively transfer cells across domains, it can not remove domainspecific effects in shared latent space $\mathcal{Z}$. To enable encoders $E_{1}(\cdot), E_{2}(\cdot)$ to eliminate domainspecific effects in $\mathcal{X}$ and $\mathcal{Y}$, we propose the latent alignment regularizer in (4) for the consistency in latent space $\mathcal{Z}$, where $\mathcal{R}_{\mathrm{LA}}=\frac{1}{q}\left\{\mathbb{E}\left[\left\|E_{1}(\mathbf{x})-E_{2}\left(G_{2}\left(E_{1}(\mathbf{x})\right)\right)\right\|_{2}^{2}\right]+\mathbb{E}\left[\left\|E_{2}(\mathbf{y})-E_{1}\left(G_{1}\left(E_{2}(\mathbf{y})\right)\right)\right\|_{2}^{2}\right]\right\}$, $q$ is the dimensionality of $\mathcal{Z}, E_{1}(\mathbf{x})$ is the latent code of a real cell $\mathbf{x} \in \mathcal{X}$ and $E_{2}\left(G_{2}\left(E_{1}(\mathbf{x})\right)\right)$ is the latent code of its transferred version, $E_{2}(\mathbf{y})$ is the latent code of a real cell $\mathbf{y} \in \mathcal{Y}$ and $E_{1}\left(G_{1}\left(E_{2}(\mathbf{y})\right)\right)$ is the latent code of its transferred version. The regularizer (4) encourages the latent codes of the same cell to be close to each other. This regularizer helps encoders $E_{1}(\cdot)$ and $E_{2}(\cdot)$ to remove domain-specific effects, such that the latent codes in $\mathcal{Z}$ preserve biological variation of cells from different domains.

Algorithm. Now we develop an alternative updating algorithm to solving the optimization problem of adversarial domain translation with the three regularizers. To efficiently solve the optimization problem, we replace the constraints (3), (4) and (5) by its Lagrange form. We introduce three regularization parameters $\lambda_{\mathrm{AE}}, \lambda_{\mathrm{LA}}$ and $\lambda_{\mathrm{cos}}$ as coefficients for the regularizers. The optimization problem of Portal is rewritten as

$$
\min _{\left\{E_{1}, G_{1}, E_{2}, G_{2}\right\}} \max _{\left\{D_{1}, D_{2}\right\}} \mathcal{L}_{\mathcal{X}}+\mathcal{L}_{\mathcal{Y}}+\lambda_{\mathrm{AE}} \mathcal{R}_{\mathrm{AE}}+\lambda_{\mathrm{LA}} \mathcal{R}_{\mathrm{LA}}+\lambda_{\mathrm{cos}} \mathcal{R}_{\mathrm{cos}}
$$

As we adopt the "logD-trick" for updating domain translation networks formed by $E_{1}(\cdot), G_{2}(\cdot)$ and $E_{2}(\cdot), G_{1}(\cdot)$, the optimization problem $(7)$ is modified accordingly as

$$
\min _{\left\{E_{1}, G_{1}, E_{2}, G_{2}\right\}} \max _{\left\{D_{1}, D_{2}\right\}} \mathcal{L}_{\mathrm{adv}}+\lambda_{\mathrm{AE}} \mathcal{R}_{\mathrm{AE}}+\lambda_{\mathrm{LA}} \mathcal{R}_{\mathrm{LA}}+\lambda_{\mathrm{cos}} \mathcal{R}_{\mathrm{cos}},
$$

where $\mathcal{L}_{\text {adv }}$ stands for the adversarial learning objective, whose value is $\mathcal{L}_{\mathcal{X}}+\mathcal{L}_{\mathcal{Y}}$ when maximizing with respect to $D_{1}(\cdot), D_{2}(\cdot)$, and it is replaced with $\mathcal{L}_{\mathcal{X}}^{\log D}+\mathcal{L}_{\mathcal{Y}}^{\log D}$ when minimizing with respect to $E_{1}(\cdot), G_{1}(\cdot), E_{2}(\cdot), G_{2}(\cdot)$.

Let the parameters of the networks $E_{1}(\cdot), E_{2}(\cdot), G_{1}(\cdot), G_{2}(\cdot), D_{1}(\cdot)$ and $D_{2}(\cdot)$ be denoted as $\theta_{E_{1}}, \theta_{E_{2}}, \theta_{G_{1}}, \theta_{G_{2}}, \theta_{D_{1}}$ and $\theta_{D_{2}}$. Then we collect the parameter sets as $\theta_{E}=\left\{\theta_{E_{1}}, \theta_{E_{2}}\right\}, \theta_{G}=$ $\left\{\theta_{G_{1}}, \theta_{G_{2}}\right\}$ and $\theta_{D}=\left\{\theta_{D_{1}}, \theta_{D_{2}}\right\}$. We use the Monte Carlo estimators to approximate expectations in Portal's objective. With a mini-batch of $2 m$ samples including $\left\{\mathbf{x}^{(1)}, \mathbf{x}^{(2)}, \cdots, \mathbf{x}^{(m)}\right\}$ from $\mathcal{X}$ 
703

and $\left\{\mathbf{y}^{(1)}, \mathbf{y}^{(2)}, \cdots, \mathbf{y}^{(m)}\right\}$ from $\mathcal{Y}$, the Monte Carlo estimators are given by

$$
\begin{aligned}
& \widehat{\mathcal{L}}_{\mathcal{X}}=\frac{1}{m} \sum_{i=1}^{m}\left[\log D_{1}\left(\mathbf{x}^{(i)}\right)+\log \left(1-D_{1}\left(G_{1}\left(E_{2}\left(\mathbf{y}^{(i)}\right)\right)\right)\right)\right], \widehat{\mathcal{L}}_{\mathcal{X}}^{\log D}=-\frac{1}{m} \sum_{i=1}^{m} \log D_{1}\left(G_{1}\left(E_{2}\left(\mathbf{y}^{(i)}\right)\right)\right), \\
& \widehat{\mathcal{L}}_{\mathcal{Y}}=\frac{1}{m} \sum_{i=1}^{m}\left[\log D_{2}\left(\mathbf{y}^{(i)}\right)+\log \left(1-D_{2}\left(G_{2}\left(E_{1}\left(\mathbf{x}^{(i)}\right)\right)\right)\right)\right], \widehat{\mathcal{L}}_{\mathcal{Y}}^{\log D}=-\frac{1}{m} \sum_{i=1}^{m} \log D_{2}\left(G_{2}\left(E_{1}\left(\mathbf{x}^{(i)}\right)\right)\right), \\
& \widehat{\mathcal{R}}_{\mathrm{AE}}=\frac{1}{m p} \sum_{i=1}^{m}\left[\left\|\mathbf{x}^{(i)}-G_{1}\left(E_{1}\left(\mathbf{x}^{(i)}\right)\right)\right\|_{2}^{2}+\left\|\mathbf{y}^{(i)}-G_{2}\left(E_{2}\left(\mathbf{y}^{(i)}\right)\right)\right\|_{2}^{2}\right], \\
& \widehat{\mathcal{R}}_{\mathrm{LA}}=\frac{1}{m q} \sum_{i=1}^{m}\left[\left\|E_{1}\left(\mathbf{x}^{(i)}\right)-E_{2}\left(G_{2}\left(E_{1}\left(\mathbf{x}^{(i)}\right)\right)\right)\right\|_{2}^{2}+\left\|E_{2}\left(\mathbf{y}^{(i)}\right)-E_{1}\left(G_{1}\left(E_{2}\left(\mathbf{y}^{(i)}\right)\right)\right)\right\|_{2}^{2}\right], \\
& \widehat{\mathcal{R}}_{\mathrm{cos}}=\frac{1}{m} \sum_{i=1}^{m}\left\{\left[1-\frac{<\mathbf{x}^{(i)}, G_{2}\left(E_{1}\left(\mathbf{x}^{(i)}\right)\right)>}{\left\|\mathbf{x}^{(i)}\right\|_{2}\left\|G_{2}\left(E_{1}\left(\mathbf{x}^{(i)}\right)\right)\right\|_{2}}\right]+\left[1-\frac{<\mathbf{y}^{(i)}, G_{1}\left(E_{2}\left(\mathbf{y}^{(i)}\right)\right)>}{\left\|\mathbf{y}^{(i)}\right\|_{2}\left\|G_{1}\left(E_{2}\left(\mathbf{y}^{(i)}\right)\right)\right\|_{2}}\right]\right\} .
\end{aligned}
$$

The implementation of Portal is summarized in Algorithm 1 .

Algorithm 1 Stochastic gradient descent training of Portal.

Require: Batch size $m$, coefficients $\lambda_{\mathrm{AE}}, \lambda_{\mathrm{LA}}$ and $\lambda_{\mathrm{cos}}$

for number of training iterations do

Sample $m$ cells $\left\{\mathbf{x}^{(1)}, \mathbf{x}^{(2)}, \cdots, \mathbf{x}^{(m)}\right\}$ from $\mathcal{X}$ and $m$ cells $\left\{\mathbf{y}^{(1)}, \mathbf{y}^{(2)}, \cdots, \mathbf{y}^{(m)}\right\}$ from $\mathcal{Y}$. Calculate $\widehat{\mathcal{L}}_{\mathcal{X}}, \widehat{\mathcal{L}}_{\mathcal{Y}}, \widehat{\mathcal{L}}_{\mathcal{X}}^{\log D}, \widehat{\mathcal{L}}_{\mathcal{Y}}^{\log D}, \widehat{\mathcal{R}}_{\mathrm{AE}}, \widehat{\mathcal{R}}_{\mathrm{LA}}$, and $\widehat{\mathcal{R}}_{\mathrm{cos}}$

Update discriminators by stochastic gradient descent with $\nabla_{\theta_{D}}\left[-\left(\widehat{\mathcal{L}}_{\mathcal{X}}+\widehat{\mathcal{L}}_{\mathcal{Y}}\right)\right]$.

Update encoders and generators simultaneously by stochastic gradient descent with

$$
\nabla_{\theta_{E}, \theta_{G}}\left(\widehat{\mathcal{L}}_{\mathcal{X}}^{\log D}+\widehat{\mathcal{L}}_{\mathcal{Y}}^{\log D}+\lambda_{\mathrm{AE}} \widehat{\mathcal{R}}_{\mathrm{AE}}+\lambda_{\mathrm{LA}} \widehat{\mathcal{R}}_{\mathrm{LA}}+\lambda_{\cos } \widehat{\mathcal{R}}_{\mathrm{cos}}\right) .
$$

\section{end for}

After training, cells from domains $\mathcal{X}$ and $\mathcal{Y}$ are encoded into $\mathcal{Z}$ to construct an integrated dataset, which can be applied to downstream analysis. In each domain, the original cells and transferred cells are also well integrated. For integration of multiple datasets, Portal can handle them incrementally, by transferring all other datasets into the domain formed by one dataset.

\section{Analysis details}

Data preprocessing. For all datasets, we used raw read or unique molecular identifier (UMI) matrices depending on the data source. We then performed standard data preprocessing for each count matrix, including log-normalization, feature selection, scaling and dimensionality reduction. For each dataset represented by a cell-by-gene count matrix, we first adopted the log-normalization, following the Seurat and Scanpy pipelines [22, 63]. For each cell, its library size was normalized to 10,000 reads. Specifically, the counts abundance of each gene was 
divided by the total counts for each cell, then multiplied by a scaling factor of 10,000 . The normalized dataset was then transformed to $\log$ scale by the function $\log (1+x)$. In order to identify a subset of features that highlight variability across individual cells, we adopted the feature selection procedure from the Seurat pipeline. For each dataset, we selected $K$ top highly variable genes ranked by dispersion with the control of means. In this paper, we used $K=4,000$ throughout all analyses except for the cross-species analysis. In the cross-species analysis, we used $K=3,000$ since the usage of a larger number of features would result in the situation that correspondence across species is dominated by the distinction (e.g., altered functions of genes annotated by the same name). For each selected variable gene, we centered and standardized its expressions across individual cells to have mean at zero and variance at one. After the above procedures, which were applied to individual datasets, we continued to preprocess data across datasets. For those datasets to be integrated, we collected genes that were identified as top highly variable genes in all of them as features for integration. We extracted the scaled data with these features from each dataset, and then concatenated them based on features to perform joint PCA. Top $p=30$ principle components were kept for all dataset as inputs to Portal. For the shared latent space, we set its dimensionality to be $q=20$ throughout all analyses.

Hyperparameter setting. Hyperparameters used in Portal are $m, t, \lambda_{\mathrm{AE}}, \lambda_{\mathrm{LA}}, \lambda_{\mathrm{cos}}$, where $m$ is the batch size used by Portal for mini-batch training; $t$ is the absolute value of boundaries for the logit of discriminator scores $\left(-t<d_{i}(\mathbf{x})<t, i=1,2\right) ; \lambda_{\mathrm{AE}}, \lambda_{\mathrm{LA}}, \lambda_{\mathrm{cos}}$ are coefficients for autoencoder consistency regularizer $\mathcal{R}_{\mathrm{AE}}$, latent alignment regularizer $\mathcal{R}_{\mathrm{LA}}$ and cosine similarity regularizer $\mathcal{R}_{\cos }$ respectively. Throughout all analyses, we set $m=500, t=5.0, \lambda_{\mathrm{AE}}=10.0$, $\lambda_{\mathrm{LA}}=10.0$. Hyperparameter $\lambda_{\text {cos }}$ was tuned within the range $[10.0,50.0]$ with interval 5.0 according to the mixing metric, where the mixing metric was designed in Seurat to evaluate how well the datasets mixed after integration. The insight into tuning $\lambda_{\cos }$ is as follows: During domain translations, there is a trade-off between preservation of similarity across domains and flexibility of modeling domain differences. Since $\mathcal{R}_{\cos }$ is designed to preserve the cosine similarity during translations, a higher value of $\lambda_{\cos }$ can enhance the cosine similarity as the cross-domain correspondence, and a lower $\lambda_{\text {cos }}$ allows domain translation networks to deal with remarkable differences between domains. Following this intuition, we empirically find out that $\lambda_{\cos }=10.0$ has a good performance when harmonizing datasets with intrinsic differences, for 
example, datasets used in cross-species analysis. For other integration tasks, $\lambda_{\cos }=20.0$ often yields reasonable results, which is adopted as the default setting in our package. Slightly better alignment results could be achieved by tuning $\lambda_{\cos }$.

Label transfer. Suppose we wish to transfer labels from domain $\mathcal{X}$ to domain $\mathcal{Y}$. As Portal produces integrated cell representations in each domain and the shared latent space, we can use any of these representations to perform label transfer. For each cell in domain $\mathcal{Y}$, we find its $k=20$-nearest neighbors among the cells in domain $\mathcal{X}$ based on the integrated result. The metric for finding nearest neighbors can be Euclidean distance in shared latent space, or cosine similarity in domains. The labels in domain $\mathcal{Y}$ are finally determined by majority voting.

Evaluation metrics. We assessed all metrics based on Portal's integration results in shared latent space $\mathcal{Z}$. We used kBET [32] for quantitative evaluation of integration approaches in terms of domain-specific effects removal ability. Firstly, kBET creates a $k$-nearest neighbour matrix. Then, $10 \%$ of the samples are chosen for hypothesis testing, where the null hypothesis is that all batches are well-mixed. For each of selected samples, kBET adopts a Pearson's $\chi^{2}$-based test to check whether the batch label distribution in its neighbourhood is similar to the global batch label distribution or not. In our experiments, we ran 100 replicates of $\mathrm{kBET}$ with 1,000 random samples, and used the median of the 100 average acceptance rates as the final result. We used the neighbourhood size following the default setting in the official implementation of kBET. To evaluate the cluster preservation performance, we used ARI and ASW. ARI measures the degree to which the two clustering results match. It outputs scores ranging from 0 to 1 , where 0 indicates that the two clustering labels are independent to each other, and 1 means that the two clustering labels are the same up to a permutation. We obtained clustering results following the Seurat clustering pipeline with its default setting, and assessed ARI by comparing identified clusters and cell type annotations. ASW is another metric to quantify cluster preservation. The silhouette width for cell $\mathbf{x}$ from cell type $C$ is defined as $(b(\mathbf{x})-a(\mathbf{x})) / \max (a(\mathbf{x}), b(\mathbf{x}))$, where $a(\mathbf{x})$ is the average distance from cell $\mathbf{x}$ to all cells from cell type $C$, and $b(\mathbf{x})$ is the minimum value of average distances from cell $\mathbf{x}$ to all cells from each cell type other than $C$. ASW lies between -1 and 1 , where a higher score means that cells are closer to cells of the same cell type, indicating good cluster preservation. Benchmarking of running time and memory usage. Standard data preprocessing such as normalization, feature selection and dimension reduction could be performed incrementally using 
mini-batches to control memory usage. In Portal's preprocessing, we adopted the incremental strategy and used a chunk size of 20,000. For example, the preprocessing of Portal took 63.4 minutes, requiring 22.0 GB peak running memory on the two mouse brain atlases datasets with 1,100,167 cells. The preprocessing time could be reduced to 37.7 minutes when the chunk size was increased to 200,000, with 36.4 GB peak running memory. Some other methods may not be able to adopt a mini-batch implementation. For the two mouse brain atlases datasets, Harmony took 17.6 minutes to finish preprocessing, but required 127.1 GB memory usage. Online iNMF performed preprocessing with mini-batches. Its default preprocessing procedure on the two mouse brain atlases datasets took 15.9 hours, with 0.6 GB memory usage. For a fair comparison, time and memory usages of data preprocessing procedures were not included in our benchmarking.

Visualization. We used the UMAP algorithm [31] for visualization of cell representations in a two-dimensional space. In all analyses, the UMAP algorithm was run with 30-nearest neighbors, minimum distance 0.3 , and correlation metric.

\section{Acknowledgements}

The authors would like to thank Camille Sophie Ezran (Stanford University), Dr. Angela Oliveira Pisco (CZ Biohub), and Dr. Hosu Sin (Stanford University) for valuable discussions. This work is supported in part by Hong Kong Research Grant Council [16101118, 24301419, 14301120, 16307818, 16301419, 16308120], the Hong Kong University of Science and Technology's startup grant [R9405,R9364], the Hong Kong University of Science and Technology Big Data for Bio Intelligence Laboratory (BDBI), the Lo Ka Chung Foundation through the Hong Kong Epigenomics Project, the Chau Hoi Shuen Foundation, the Chinese University of Hong Kong direct grants [4053360, 4053423, 4053476], the Chinese University of Hong Kong startup grant [4930181], the Chinese University of Hong Kong's Project Impact Enhancement Fund (PIEF) and Science Faculty's Collaborative Research Impact Matching Scheme (CRIMS), the East China Normal University startup grant, the Shanghai Sailing Program. The computational task for this work was partially performed using the X-GPU cluster supported by the RGC Collaborative Research Fund: C6021-19EF. 


\section{Data availability}

All data used in this work are publicly available through online sources.

- Mouse Brain cells from Saunders et al [8] (http://dropviz.org).

- Mouse Brain cells from Zeisel et al [9] (http://mousebrain.org/downloads.html).

- Mouse Brain cells from Rosenberg et al [43] (GSE110823).

- Mouse cell atlas from the Tabula Muris Consortium [7] (https://figshare.com/project $/$ Tabula_Muris_Transcriptomic_characterization_of_20_organs_and_tissues_from_ Mus_musculus_at_single_cell_resolution/27733).

- Mouse lemur cell atlas from the Tabula Microcebus Consortium (https://figshare com/projects/Tabula_Microcebus/112227).

- Mouse spermatogenesis cells from Ernst et al [47] (https://www.ebi.ac.uk/arrayexpress/ experiments/E-MTAB-6946/).

- Human spermatogenesis cells from Shami et al [16] (GSE142585).

- Macaque spermatogenesis cells from Shami et al [16] (GSE142585).

\section{Code availability}

Portal software is available at https://github.com/YangLabHKUST/Portal. 


\section{References}

[1] Alexandra-Chloé Villani, Rahul Satija, Gary Reynolds, Siranush Sarkizova, Karthik Shekhar, James Fletcher, Morgane Griesbeck, Andrew Butler, Shiwei Zheng, Suzan Lazo, et al. Single-cell RNA-seq reveals new types of human blood dendritic cells, monocytes, and progenitors. Science, 356(6335), 2017.

[2] Maria Brbić, Marinka Zitnik, Sheng Wang, Angela O Pisco, Russ B Altman, Spyros Darmanis, and Jure Leskovec. Mars: discovering novel cell types across heterogeneous single-cell experiments. Nature methods, 17(12):1200-1206, 2020.

[3] Giovanni Iacono, Ramon Massoni-Badosa, and Holger Heyn. Single-cell transcriptomics unveils gene regulatory network plasticity. Genome biology, 20(1):1-20, 2019.

[4] Anna SE Cuomo, Daniel D Seaton, Davis J McCarthy, Iker Martinez, Marc Jan Bonder, Jose Garcia-Bernardo, Shradha Amatya, Pedro Madrigal, Abigail Isaacson, Florian Buettner, et al. Single-cell RNA-sequencing of differentiating iPS cells reveals dynamic genetic effects on gene expression. Nature communications, 11(1):1-14, 2020.

[5] Barbara Treutlein, Doug G Brownfield, Angela R Wu, Norma F Neff, Gary L Mantalas, F Hernan Espinoza, Tushar J Desai, Mark A Krasnow, and Stephen R Quake. Reconstructing lineage hierarchies of the distal lung epithelium using single-cell RNA-seq. Nature, 509(7500):371-375, 2014.

[6] Xiaojie Qiu, Qi Mao, Ying Tang, Li Wang, Raghav Chawla, Hannah A Pliner, and Cole Trapnell. Reversed graph embedding resolves complex single-cell trajectories. Nature methods, 14(10):979-982, 2017.

[7] Tabula Muris Consortium et al. Single-cell transcriptomics of 20 mouse organs creates a Tabula Muris. Nature, 562(7727):367-372, 2018.

[8] Arpiar Saunders, Evan Z Macosko, Alec Wysoker, Melissa Goldman, Fenna M Krienen, Heather de Rivera, Elizabeth Bien, Matthew Baum, Laura Bortolin, Shuyu Wang, et al. Molecular diversity and specializations among the cells of the adult mouse brain. Cell, 174(4):1015-1030, 2018. 
[9] Amit Zeisel, Hannah Hochgerner, Peter Lönnerberg, Anna Johnsson, Fatima Memic, Job Van Der Zwan, Martin Häring, Emelie Braun, Lars E Borm, Gioele La Manno, et al. Molecular architecture of the mouse nervous system. Cell, 174(4):999-1014, 2018.

[10] Hoa Thi Nhu Tran, Kok Siong Ang, Marion Chevrier, Xiaomeng Zhang, Nicole Yee Shin Lee, Michelle Goh, and Jinmiao Chen. A benchmark of batch-effect correction methods for single-cell RNA sequencing data. Genome biology, 21(1):1-32, 2020.

[11] Oliver Stegle, Sarah A Teichmann, and John C Marioni. Computational and analytical challenges in single-cell transcriptomics. Nature Reviews Genetics, 16(3):133-145, 2015.

[12] Monika Litviňuková, Carlos Talavera-López, Henrike Maatz, Daniel Reichart, Catherine L Worth, Eric L Lindberg, Masatoshi Kanda, Krzysztof Polanski, Matthias Heinig, Michael Lee, et al. Cells of the adult human heart. Nature, 588(7838):466-472, 2020.

[13] Kyle J Travaglini, Ahmad N Nabhan, Lolita Penland, Rahul Sinha, Astrid Gillich, Rene V Sit, Stephen Chang, Stephanie D Conley, Yasuo Mori, Jun Seita, et al. A molecular cell atlas of the human lung from single-cell RNA sequencing. Nature, 587(7835):619-625, 2020 .

[14] Blue B Lake, Simone Codeluppi, Yun C Yung, Derek Gao, Jerold Chun, Peter V Kharchenko, Sten Linnarsson, and Kun Zhang. A comparative strategy for single-nucleus and single-cell transcriptomes confirms accuracy in predicted cell-type expression from nuclear RNA. Scientific reports, 7(1):1-8, 2017.

[15] Haojia Wu, Yuhei Kirita, Erinn L Donnelly, and Benjamin D Humphreys. Advantages of single-nucleus over single-cell RNA sequencing of adult kidney: rare cell types and novel cell states revealed in fibrosis. Journal of the American Society of Nephrology, 30(1):23-32, 2019.

[16] Adrienne Niederriter Shami, Xianing Zheng, Sarah K Munyoki, Qianyi Ma, Gabriel L Manske, Christopher D Green, Meena Sukhwani, Kyle E Orwig, Jun Z Li, and Saher Sue Hammoud. Single-cell RNA sequencing of human, macaque, and mouse testes uncovers conserved and divergent features of mammalian spermatogenesis. Developmental cell, 54(4):529-547, 2020. 
[17] Aviv Regev, Sarah A Teichmann, Eric S Lander, Ido Amit, Christophe Benoist, Ewan Birney, Bernd Bodenmiller, Peter Campbell, Piero Carninci, Menna Clatworthy, et al. Science forum: the human cell atlas. elife, 6:e27041, 2017.

[18] Xiaoping Han, Renying Wang, Yincong Zhou, Lijiang Fei, Huiyu Sun, Shujing Lai, Assieh Saadatpour, Ziming Zhou, Haide Chen, Fang Ye, et al. Mapping the mouse cell atlas by microwell-seq. Cell, 172(5):1091-1107, 2018.

[19] Si Wang, Yuxuan Zheng, Jingyi Li, Yang Yu, Weiqi Zhang, Moshi Song, Zunpeng Liu, Zheying Min, Huifang Hu, Ying Jing, et al. Single-cell transcriptomic atlas of primate ovarian aging. Cell, 180(3):585-600, 2020.

[20] Shuai Ma, Shuhui Sun, Jiaming Li, Yanling Fan, Jing Qu, Liang Sun, Si Wang, Yiyuan Zhang, Shanshan Yang, Zunpeng Liu, et al. Single-cell transcriptomic atlas of primate cardiopulmonary aging. Cell research, 31(4):415-432, 2021.

[21] Ilya Korsunsky, Nghia Millard, Jean Fan, Kamil Slowikowski, Fan Zhang, Kevin Wei, Yuriy Baglaenko, Michael Brenner, Po-ru Loh, and Soumya Raychaudhuri. Fast, sensitive and accurate integration of single-cell data with Harmony. Nature methods, 16(12):1289-1296, 2019.

[22] Tim Stuart, Andrew Butler, Paul Hoffman, Christoph Hafemeister, Efthymia Papalexi, William M Mauck III, Yuhan Hao, Marlon Stoeckius, Peter Smibert, and Rahul Satija. Comprehensive integration of single-cell data. Cell, 177(7):1888-1902, 2019.

[23] Chao Gao, Jialin Liu, April R Kriebel, Sebastian Preissl, Chongyuan Luo, Rosa Castanon, Justin Sandoval, Angeline Rivkin, Joseph R Nery, Margarita M Behrens, et al. Iterative single-cell multi-omic integration using online learning. Nature Biotechnology, pages 1-8, 2021.

[24] Laleh Haghverdi, Aaron TL Lun, Michael D Morgan, and John C Marioni. Batch effects in single-cell RNA-sequencing data are corrected by matching mutual nearest neighbors. Nature biotechnology, 36(5):421-427, 2018.

[25] Brian Hie, Bryan Bryson, and Bonnie Berger. Efficient integration of heterogeneous single-cell transcriptomes using Scanorama. Nature biotechnology, 37(6):685-691, 2019. 

available under aCC-BY-NC-ND 4.0 International license.

[26] Krzysztof Polański, Matthew D Young, Zhichao Miao, Kerstin B Meyer, Sarah A Teichmann, and Jong-Eun Park. BBKNN: fast batch alignment of single cell transcriptomes. Bioinformatics, 36(3):964-965, 2020.

[27] Ian Goodfellow, Jean Pouget-Abadie, Mehdi Mirza, Bing Xu, David Warde-Farley, Sherjil Ozair, Aaron Courville, and Yoshua Bengio. Generative adversarial nets. In Advances in Neural Information Processing Systems, pages 2672-2680, 2014.

[28] Jun-Yan Zhu, Taesung Park, Phillip Isola, and Alexei A Efros. Unpaired image-toimage translation using cycle-consistent adversarial networks. In Proceedings of the IEEE International Conference on Computer Vision, pages 2223-2232, 2017.

[29] Ming-Yu Liu, Thomas Breuel, and Jan Kautz. Unsupervised image-to-image translation networks. In Advances in Neural Information Processing Systems, pages 700-708, 2017.

[30] Yunjey Choi, Minje Choi, Munyoung Kim, Jung-Woo Ha, Sunghun Kim, and Jaegul Choo. Stargan: Unified generative adversarial networks for multi-domain image-to-image translation. In Proceedings of the IEEE conference on Computer Vision and Pattern Recognition, pages 8789-8797, 2018.

[31] Leland McInnes, John Healy, Nathaniel Saul, and Lukas Grossberger. UMAP: Uniform manifold approximation and projection. The Journal of Open Source Software, 3(29):861, 2018.

[32] Maren Büttner, Zhichao Miao, F Alexander Wolf, Sarah A Teichmann, and Fabian J Theis. A test metric for assessing single-cell RNA-seq batch correction. Nature methods, 16(1):43-49, 2019.

[33] Joshua D Welch, Velina Kozareva, Ashley Ferreira, Charles Vanderburg, Carly Martin, and Evan Z Macosko. Single-cell multi-omic integration compares and contrasts features of brain cell identity. Cell, 177(7):1873-1887, 2019.

[34] Vincent D Blondel, Jean-Loup Guillaume, Renaud Lambiotte, and Etienne Lefebvre. Fast unfolding of communities in large networks. Journal of statistical mechanics: theory and experiment, 2008(10):P10008, 2008. 
[35] Jeanette Baran-Gale, Tamir Chandra, and Kristina Kirschner. Experimental design for single-cell RNA sequencing. Briefings in functional genomics, 17(4):233-239, 2018.

[36] Joey Schyns, Fabrice Bureau, and Thomas Marichal. Lung interstitial macrophages: past, present, and future. Journal of immunology research, 2018, 2018.

[37] Tabula Microcebus Consortium. https://tabula-microcebus.ds.czbiohub.org.

[38] Ting Xie, Yizhou Wang, Nan Deng, Guanling Huang, Forough Taghavifar, Yan Geng, Ningshan Liu, Vrishika Kulur, Changfu Yao, Peter Chen, et al. Single-cell deconvolution of fibroblast heterogeneity in mouse pulmonary fibrosis. Cell reports, 22(13):3625-3640, 2018.

[39] Alan Selewa, Ryan Dohn, Heather Eckart, Stephanie Lozano, Bingqing Xie, Eric Gauchat, Reem Elorbany, Katherine Rhodes, Jonathan Burnett, Yoav Gilad, et al. Systematic comparison of high-throughput single-cell and single-nucleus transcriptomes during cardiomyocyte differentiation. Scientific reports, 10(1):1-13, 2020.

[40] Anne-Marie Galow, Markus Wolfien, Paula Müller, Madeleine Bartsch, Ronald M Brunner, Andreas Hoeflich, Olaf Wolkenhauer, Robert David, and Tom Goldammer. Integrative cluster analysis of whole hearts reveals proliferative cardiomyocytes in adult mice. Cells, $9(5): 1144,2020$.

[41] Blue B Lake, Rizi Ai, Gwendolyn E Kaeser, Neeraj S Salathia, Yun C Yung, Rui Liu, Andre Wildberg, Derek Gao, Ho-Lim Fung, Song Chen, et al. Neuronal subtypes and diversity revealed by single-nucleus RNA sequencing of the human brain. Science, 352(6293):15861590, 2016.

[42] Ricard Argelaguet, Anna SE Cuomo, Oliver Stegle, and John C Marioni. Computational principles and challenges in single-cell data integration. Nature Biotechnology, pages 1-14, 2021.

[43] Alexander B Rosenberg, Charles M Roco, Richard A Muscat, Anna Kuchina, Paul Sample, Zizhen Yao, Lucas T Graybuck, David J Peeler, Sumit Mukherjee, Wei Chen, et al. Singlecell profiling of the developing mouse brain and spinal cord with split-pool barcoding. Science, 360(6385):176-182, 2018. 
[44] Chunmei Cui, Yuan Zhou, and Qinghua Cui. Defining the functional divergence of orthologous genes between human and mouse in the context of miRNA regulation. Briefings in Bioinformatics, pages 1477-4054, 2021.

[45] Christopher Daniel Green, Qianyi Ma, Gabriel L Manske, Adrienne Niederriter Shami, Xianing Zheng, Simone Marini, Lindsay Moritz, Caleb Sultan, Stephen J Gurczynski, Bethany B Moore, et al. A comprehensive roadmap of murine spermatogenesis defined by single-cell RNA-seq. Developmental cell, 46(5):651-667, 2018.

[46] Brian P Hermann, Keren Cheng, Anukriti Singh, Lorena Roa-De La Cruz, Kazadi N Mutoji, I-Chung Chen, Heidi Gildersleeve, Jake D Lehle, Max Mayo, Birgit Westernströer, et al. The mammalian spermatogenesis single-cell transcriptome, from spermatogonial stem cells to spermatids. Cell reports, 25(6):1650-1667, 2018.

[47] Christina Ernst, Nils Eling, Celia P Martinez-Jimenez, John C Marioni, and Duncan T Odom. Staged developmental mapping and X chromosome transcriptional dynamics during mouse spermatogenesis. Nature communications, 10(1):1-20, 2019.

[48] Xianzhong Lau, Prabhakaran Munusamy, Mor Jack Ng, and Mahesh Sangrithi. Single-cell RNA sequencing of the Cynomolgus macaque testis reveals conserved transcriptional profiles during mammalian spermatogenesis. Developmental Cell, 54(4):548-566, 2020.

[49] Ho-Su Sin and Satoshi H Namekawa. The great escape: Active genes on inactive sex chromosomes and their evolutionary implications. Epigenetics, 8(9):887-892, 2013.

[50] Jennifer F Hughes and David C Page. The biology and evolution of mammalian Y chromosomes. Annual review of genetics, 49:507-527, 2015.

[51] Satoshi H Namekawa and Jeannie T Lee. XY and ZW: is meiotic sex chromosome inactivation the rule in evolution? PLoS genetics, 5(5):e1000493, 2009.

[52] Jeffrey M Cloutier and James MA Turner. Meiotic sex chromosome inactivation. Current Biology, 20(22):R962-R963, 2010.

[53] Erica L Larson, Emily EK Kopania, and Jeffrey M Good. Spermatogenesis and the evolution of mammalian sex chromosomes. Trends in Genetics, 34(9):722-732, 2018. 
[54] Jennifer F Hughes, Helen Skaletsky, Laura G Brown, Tatyana Pyntikova, Tina Graves, Robert S Fulton, Shannon Dugan, Yan Ding, Christian J Buhay, Colin Kremitzki, et al. Strict evolutionary conservation followed rapid gene loss on human and rhesus Y chromosomes. Nature, 483(7387):82-86, 2012.

[55] Jennifer F Hughes, Helen Skaletsky, Tatyana Pyntikova, Tina A Graves, Saskia KM Van Daalen, Patrick J Minx, Robert S Fulton, Sean D McGrath, Devin P Locke, Cynthia Friedman, et al. Chimpanzee and human Y chromosomes are remarkably divergent in structure and gene content. Nature, 463(7280):536-539, 2010.

[56] Polly Campbell, Jeffrey M Good, and Michael W Nachman. Meiotic sex chromosome inactivation is disrupted in sterile hybrid male house mice. Genetics, 193(3):819-828, 2013.

[57] Ho-Su Sin, Yosuke Ichijima, Eitetsu Koh, Mikio Namiki, and Satoshi H Namekawa. Human postmeiotic sex chromatin and its impact on sex chromosome evolution. Genome research, $22(5): 827-836,2012$.

[58] Malte D Luecken, Maren Büttner, Kridsadakorn Chaichoompu, Anna Danese, Marta Interlandi, Michaela Fee Mueller, Daniel C Strobl, Luke Zappia, Martin Dugas, Maria Colomé-Tatché, et al. Benchmarking atlas-level data integration in single-cell genomics. BioRxiv, 2020.

[59] Karren Dai Yang, Anastasiya Belyaeva, Saradha Venkatachalapathy, Karthik Damodaran, Abigail Katcoff, Adityanarayanan Radhakrishnan, GV Shivashankar, and Caroline Uhler. Multi-domain translation between single-cell imaging and sequencing data using autoencoders. Nature Communications, 12(1):1-10, 2021.

[60] Dongfang Wang, Siyu Hou, Lei Zhang, Xiliang Wang, Baolin Liu, and Zemin Zhang. imap: integration of multiple single-cell datasets by adversarial paired transfer networks. Genome biology, 22(1):1-24, 2021.

[61] Martin Arjovsky and Léon Bottou. Towards principled methods for training generative adversarial networks. In $I C L R, 2017$.

[62] Sean C Bendall, Kara L Davis, El-ad David Amir, Michelle D Tadmor, Erin F Simonds, Tiffany J Chen, Daniel K Shenfeld, Garry P Nolan, and Dana Pe'er. Single-cell trajectory 
bioRxiv preprint doi: https://doi.org/10.1101/2021.11.16.468892; this version posted November 19,2021 . The copyright holder for this preprint (which was not certified by peer review) is the author/funder, who has granted bioRxiv a license to display the preprint in perpetuity. It is made available under aCC-BY-NC-ND 4.0 International license.

1015

1016

1017

1018

detection uncovers progression and regulatory coordination in human B cell development. Cell, 157(3):714-725, 2014.

[63] F Alexander Wolf, Philipp Angerer, and Fabian J Theis. SCANPY: large-scale single-cell gene expression data analysis. Genome biology, 19(1):1-5, 2018. 


\section{Supplementary Information}

\section{Investigation of the role of each component in Portal}

In this section, we investigate the role of each component in Portal. The optimization problem solved by Portal is

$$
\min _{\left\{E_{1}, G_{1}, E_{2}, G_{2}\right\}} \max _{\left\{D_{1}, D_{2}\right\}} \mathcal{L}_{\mathrm{adv}}+\lambda_{\mathrm{cos}} \mathcal{R}_{\mathrm{cos}}+\lambda_{\mathrm{LA}} \mathcal{R}_{\mathrm{LA}}+\lambda_{\mathrm{AE}} \mathcal{R}_{\mathrm{AE}}
$$

where $E_{1}(\cdot)$ and $E_{2}(\cdot)$ are encoder networks; $G_{1}(\cdot)$ and $G_{2}(\cdot)$ are generator networks; $D_{1}(\cdot)$ and $D_{2}(\cdot)$ are discriminator networks; $\mathcal{L}_{\text {adv }}$ stands for the adversarial learning objective, whose value is $\mathcal{L}_{\mathcal{X}}+\mathcal{L}_{\mathcal{Y}}$ when maximizing with respect to $D_{1}(\cdot), D_{2}(\cdot)$, and it is replaced with $\mathcal{L}_{\mathcal{X}}^{\log D}+\mathcal{L}_{\mathcal{Y}}^{\log D}$ when minimizing with respect to $E_{1}(\cdot), G_{1}(\cdot), E_{2}(\cdot), G_{2}(\cdot)$ according to the "logDtrick"; $\mathcal{R}_{\mathrm{cos}}=\mathcal{R}_{\cos }\left(E_{1}, G_{1}, E_{2}, G_{2}\right)$ is a regularizer for the cosine similarity correspondence cross domains; $\mathcal{R}_{\mathrm{LA}}=\mathcal{R}_{\mathrm{LA}}\left(E_{1}, G_{1}, E_{2}, G_{2}\right)$ is a regularizer for the alignment consistency in the latent space; $\mathcal{R}_{\mathrm{AE}}=\mathcal{R}_{\mathrm{AE}}\left(E_{1}, G_{1}, E_{2}, G_{2}\right)$ is a regularizer for the autoencoder consistency; $\lambda_{\mathrm{cos}}, \lambda_{\mathrm{LA}}, \lambda_{\mathrm{AE}}$ are coefficients for the three regularizers respectively. To demonstrate the roles of the objective function $\mathcal{L}_{\text {adv }}$ and three regularizers $\left(\mathcal{R}_{\mathrm{cos}}, \mathcal{R}_{\mathrm{LA}}\right.$, and $\left.\mathcal{R}_{\mathrm{AE}}\right)$, we rewrite the optimization problem (S1) as

$$
\min _{\left\{E_{1}, G_{1}, E_{2}, G_{2}\right\}} \max _{\left\{D_{1}, D_{2}\right\}} \lambda_{\mathrm{adv}} \mathcal{L}_{\mathrm{adv}}+\lambda_{\mathrm{cos}} \mathcal{R}_{\mathrm{cos}}+\lambda_{\mathrm{LA}} \mathcal{R}_{\mathrm{LA}}+\lambda_{\mathrm{AE}} \mathcal{R}_{\mathrm{AE}}
$$

with $\lambda_{\text {adv }}$ set to 1.0 in Portal's algorithm. Based on (S2), we are able to study on the impact of each component of Portal by manually setting the corresponding coefficient to zero, and then compare its performance with that of the standard algorithm empirically. Recall that the discriminators are designed to deal with domain-unique cell types by discriminator score thresholding. In this section, we also experimentally verify the effectiveness of such design. Here we took mouse mammary gland scRNA-seq atlas from the Tabula Muris consortium as an example. In the mouse mammary gland data, 4,481 cells were profiled by 10X Genomics (10X), and 2,405 cells were profiled by SMART-seq2 (SS2). With these two datasets, we investigate the role of each component in Portal.

Role of objective function $\mathcal{L}_{\text {adv }}$. The objective function $\mathcal{L}_{\text {adv }}$ plays an essential role in learning effective domain translation across different datasets. To demonstrate the importance of adversarial training using $\mathcal{L}_{\text {adv }}$, we removed it from Portal by setting $\lambda_{\text {adv }}$ to zero, then applied this version of Portal (Portal $\left.\left(\lambda_{\mathrm{adv}}=0\right)\right)$ to integrate mouse mammary gland datasets. 

available under aCC-BY-NC-ND 4.0 International license.

Comparison between integration results obtained by Portal (Fig. S2a) and Portal $\left(\lambda_{\text {adv }}=0\right)$ )

(Fig. S2b) confirmed that cells from different datasets could not be well mixed without the objective function.

Role of regularizer $\mathcal{R}_{\text {cos }}$. Regularizer $\mathcal{R}_{\text {cos }}$ helps to establish reliable alignment between different domains. It guides domain translation networks to find correspondence of the same cell type across domains. To confirm this, we fixed $\lambda_{\text {cos }}$ in (S2) as zero to remove $\mathcal{R}_{\text {cos }}$ from Portal, and we denoted this version of Portal as Portal $\left(\lambda_{\cos }=0\right)$. After applying Portal $\left(\lambda_{\cos }=0\right)$, cells from the two datasets were well mixed, however, the obtained alignment between these datasets was problematic. For example, basal cells from SS2 dataset were incorrectly aligned with $\mathrm{B}$ cells and $\mathrm{T}$ cells from 10X dataset (Fig. S1 ). In contrast, the standard version of Portal built the alignment correctly (Fig. S1a). The difference between results obtained by Portal and Portal $\left(\lambda_{\mathrm{cos}}=0\right)$ verified the usefulness of $\mathcal{R}_{\mathrm{cos}}$ to establish robust correspondence between datasets.

Role of regularizer $\mathcal{R}_{\mathrm{LA}}$. Regularizer $\mathcal{R}_{\mathrm{LA}}$ is introduced to impose the consistency constraint for latent representations of cells. It is helpful to remove domain-specific effects in the latent space. To demonstrate the effectiveness of $\mathcal{R}_{\mathrm{LA}}$, we set $\lambda_{\mathrm{LA}}=0$. For Portal $\left(\lambda_{\mathrm{LA}}=0\right)$, the learned representation in the latent space showed a poor alignment of two datasets (Fig. S1d). This result indicated that the learned representation in the latent space would not be a valid integration result without adopting regularizer $\mathcal{R}_{\mathrm{LA}}$.

Role of regularizer $\mathcal{R}_{\mathrm{AE}} \cdot\left\{E_{1}(\cdot), G_{1}(\cdot)\right\}$ and $\left\{E_{2}(\cdot), G_{2}(\cdot)\right\}$ form two autoencoder structures in Portal's framework, $\mathcal{R}_{\mathrm{AE}}$ is hence introduced for regularizing autoencoder consistency. Here we set $\lambda_{\mathrm{AE}}=0$ to evaluate the role of $\mathcal{R}_{\mathrm{AE}}$ with Portal $\left(\lambda_{\mathrm{AE}}=0\right)$. Comparison between results obtained by Portal (Fig. S1a) and Portal $\left(\lambda_{\mathrm{AE}}=0\right)$ (Fig. S1e) indicated that $\mathcal{R}_{\mathrm{AE}}$ was useful to improve the accuracy of Portal's results by imposing the consistency between encoders and generators.

Role of discriminator score thresholding. The discriminator score thresholding in Portal is a tailored design for single-cell integration tasks. With such design, Portal does not force the alignment of domain-unique cell types, preventing overcorrection of domain-specific effects. To illustrate the role of the discriminators, we used the same mouse mammary gland data. We manually removed all basal cells from the $10 \mathrm{X}$ dataset and thereby basal cell type became a domain-unique cell type in the SS2 dataset. We applied standard Portal and Portal without 
discriminator score thresholding (denoted as "Portal w/o $D$ score thresholding") for integration.

The results in Fig. S2 indicated that, without discriminator score thresholding, Portal could not retain the identity of basal cells in the SS2 dataset, and incorrectly aligned them with T cells and B cells in the 10X dataset.

\section{Supplementary Information: Figures}
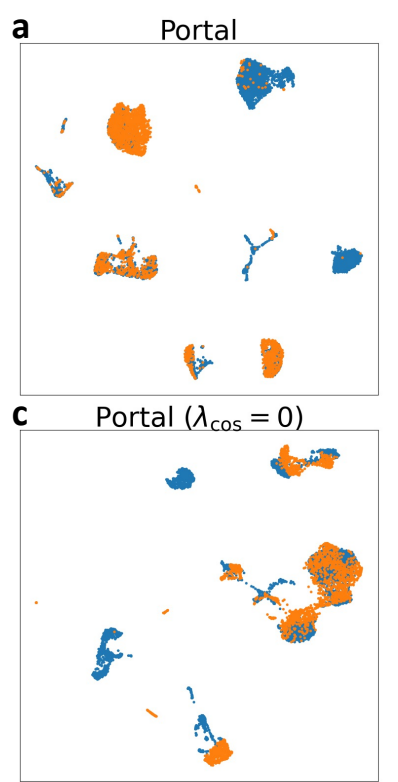

e

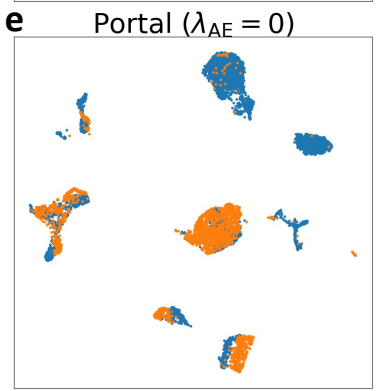

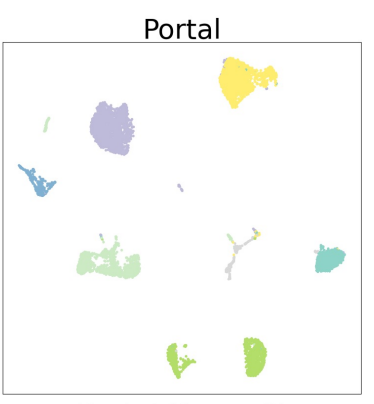

Portal $\left(\lambda_{\text {cos }}=0\right)$

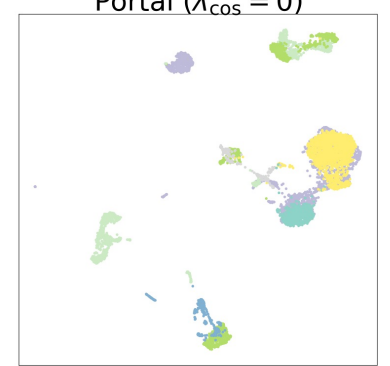

Portal $\left(\lambda_{\mathrm{AE}}=0\right)$

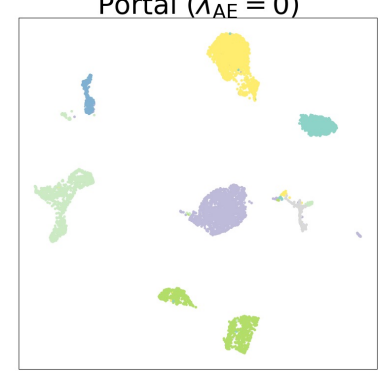

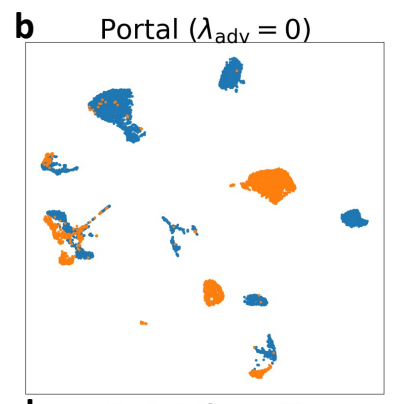

d

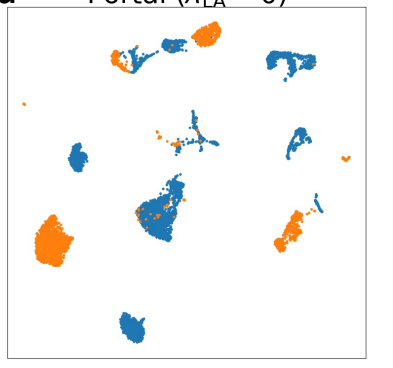

Method

- $10 \mathrm{X}$

- SS2
Cell type

- B cell

- Basal cell

- Endothelial cell

- Luminal epithelial cell of mammary gland

- Macrophage

Stromal cell

T cell

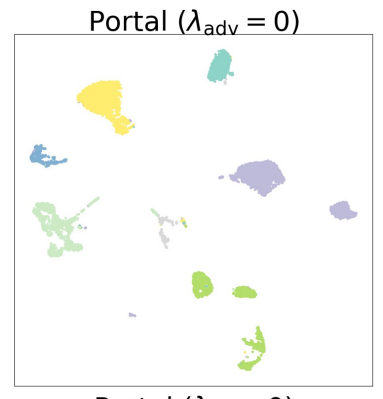

Portal $\left(\lambda_{L A}=0\right)$

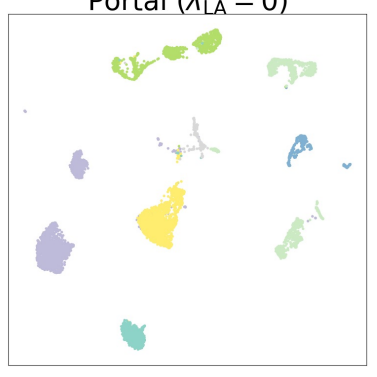

Figure S1: Investigation of the roles of objective function $\mathcal{L}_{\text {adv }}$ and three regularizers $\mathcal{R}_{\text {cos }}, \mathcal{R}_{\mathrm{LA}}$ and $\mathcal{R}_{\mathrm{AE}}$ in Portal. We used the mouse mammary gland scRNA-seq datasets from Tabluma Muris Consortium in this study. a. We applied Portal to integrate the two datasets as a baseline. b-e. Then we fixed $\lambda_{\mathrm{adv}}, \lambda_{\mathrm{cos}}, \lambda_{\mathrm{LA}}, \lambda_{\mathrm{AE}}$ in (S2) at zero to evaluate the effectiveness of $\mathcal{L}_{\text {adv }}, \mathcal{R}_{\text {cos }}, \mathcal{R}_{\mathrm{LA}}$ and $\mathcal{R}_{\mathrm{AE}}$, respectively. Clearly, each component of Poral plays its important role in data integration. 
a

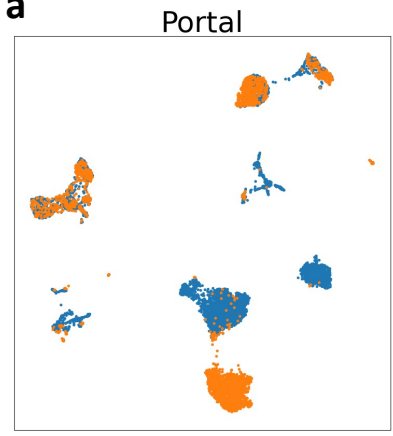

Method

- 10X

- SS2

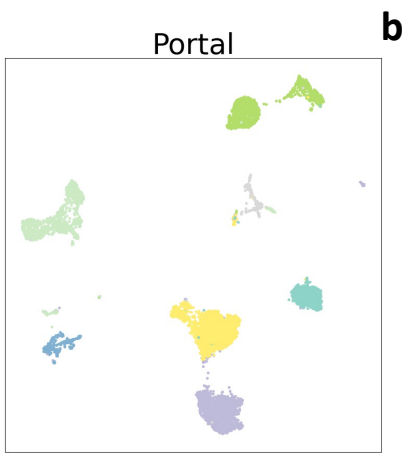

b

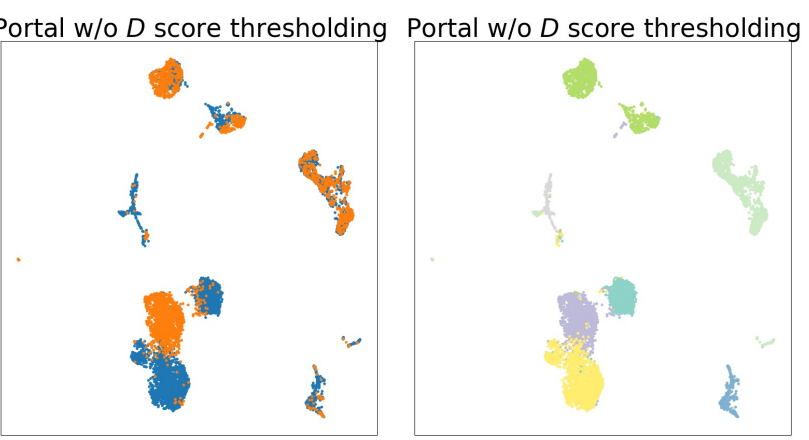

Cell type

- B cell

- Basal cell

- Endothelial cell

- Macrophage

- Stromal cell

- T cell

- Luminal epithelial cell of mammary gland

Figure S2: Investigation of the role of discriminator score thresholding in Portal. We used the same mouse mammary gland data from Tabluma Muris Consortium, and removed all basal cells from the 10X dataset to make basal cell a domain-unique cell type in the SS2 dataset. a. We applied Portal to integrating the two datasets as a baseline. b. We removed discriminator score thresholding in Portal to integrate the two datasets. 
a
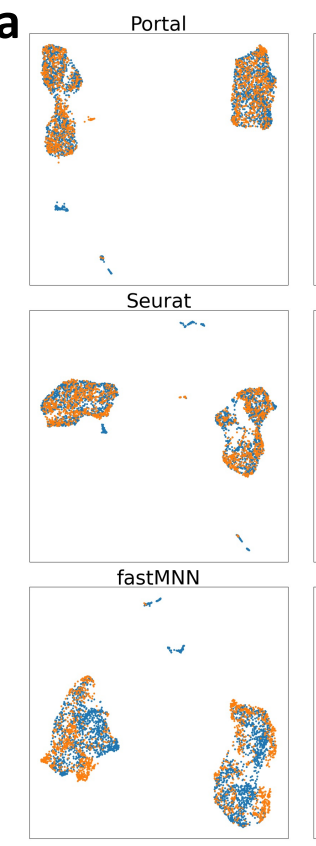

BBKNN

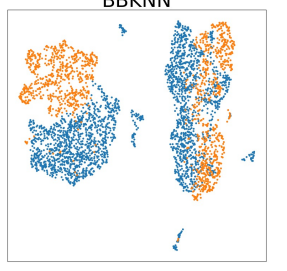

Portal
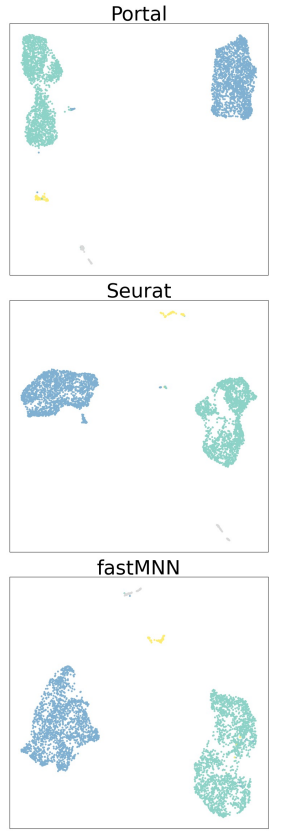

BBKNN

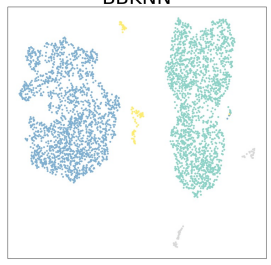

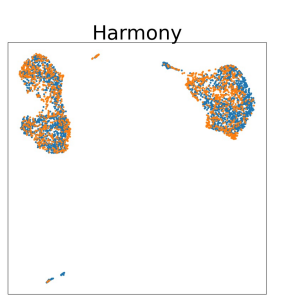

Online iNMF
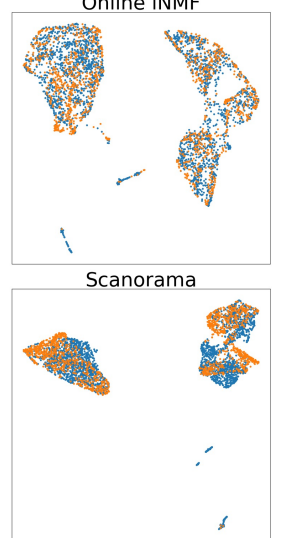

Method

- 10X

- SS2

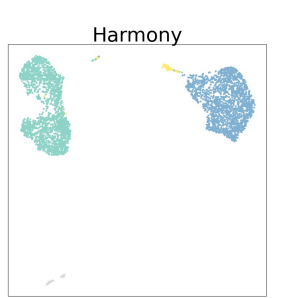

Online iNMF

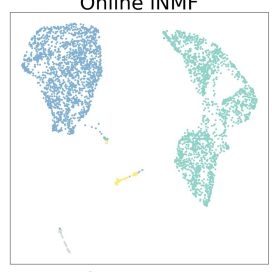

Scanorama

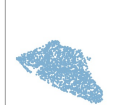

Cell type

- Bladder cell

- Bladder urothelial cell

Endothelial cell

Leukocyte b
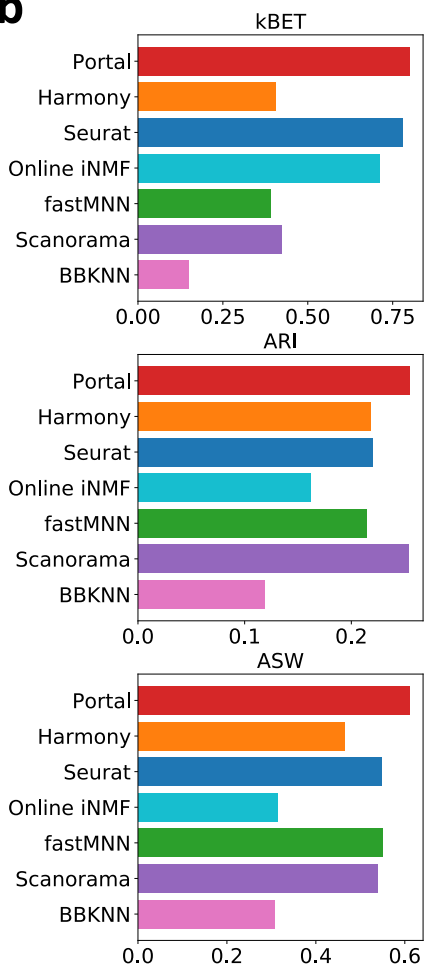

Figure S3: Comparison of integration methods based on mouse bladder data. a. UMAP plots colored by profiling methods and cell types. b. Alignment (kBET) and cluster preservation performance (ARI and ASW) evaluated using the mouse bladder data. 
a
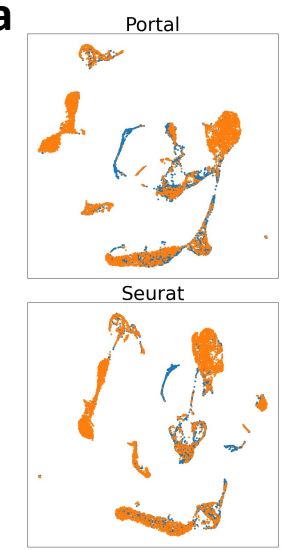

fastMNN
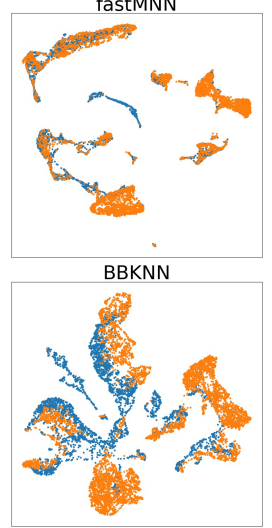

Portal

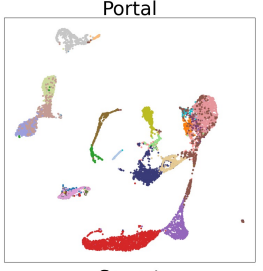

Seurat
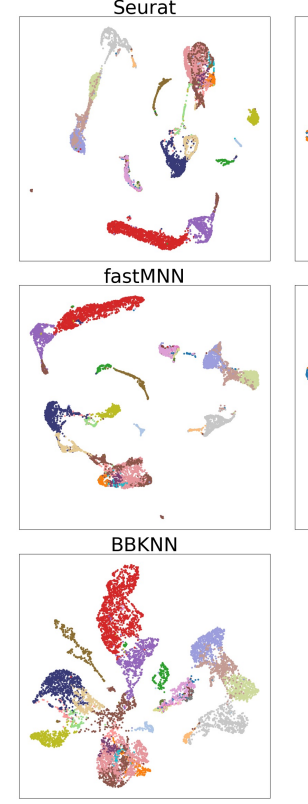
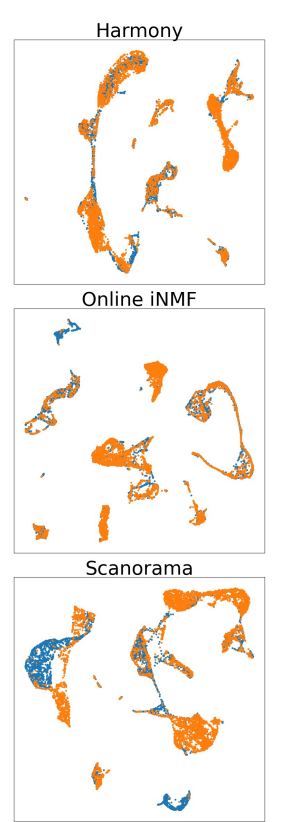

Method Cell type

- 10X

- B cell

- Basophil

- Common lymphoid progenitor

- Early pro-b cell

- Erythroblast

- Fraction a pre-pro b cell

- Granulocyte

- Granulocyte monocyte progenitor cell

- Granulocytopoietic cell

- Hematopoietic precursor cell

- Immature b cell

- Immature natural killer cell

- Immature nk t cell

- Immature t cell b
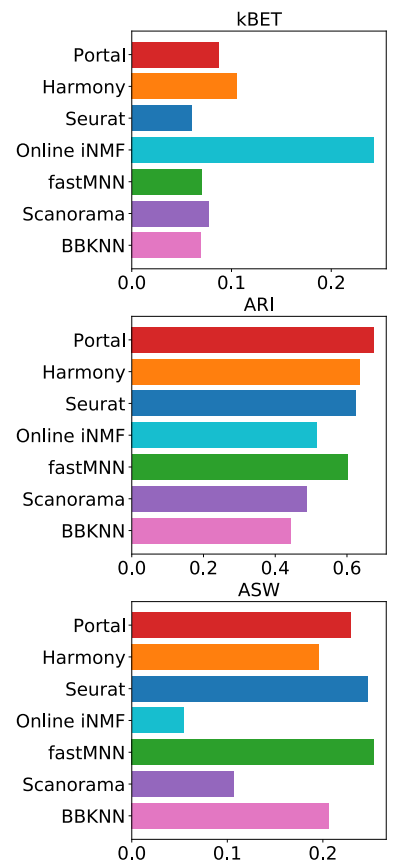

- Late pro-b cell

- Macrophage

- Mature natural killer cell

- Megakaryocyte-erythroid progenitor cell

- Monocyte

- Naive b cell

- Pre-natural killer cell

- Precursor b cell

- Proerythroblast

- Promonocyte

- Regulatory t cell

- Slamf1-negative multipotent progenitor cell

- Slamf1-positive multipotent progenitor cell

- T cell

Figure S4: Comparison of integration methods based on mouse marrow data. a. UMAP plots colored by methods and cell types. b. Alignment (kBET) and cluster preservation performance (ARI and ASW) evaluated using the mouse marrow data. 
a
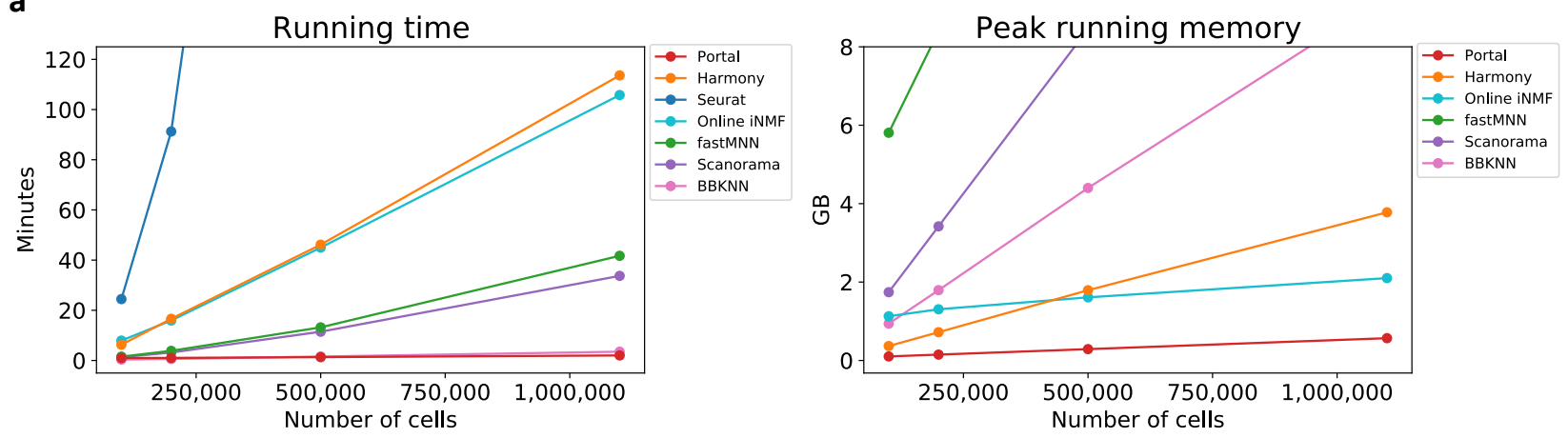

b
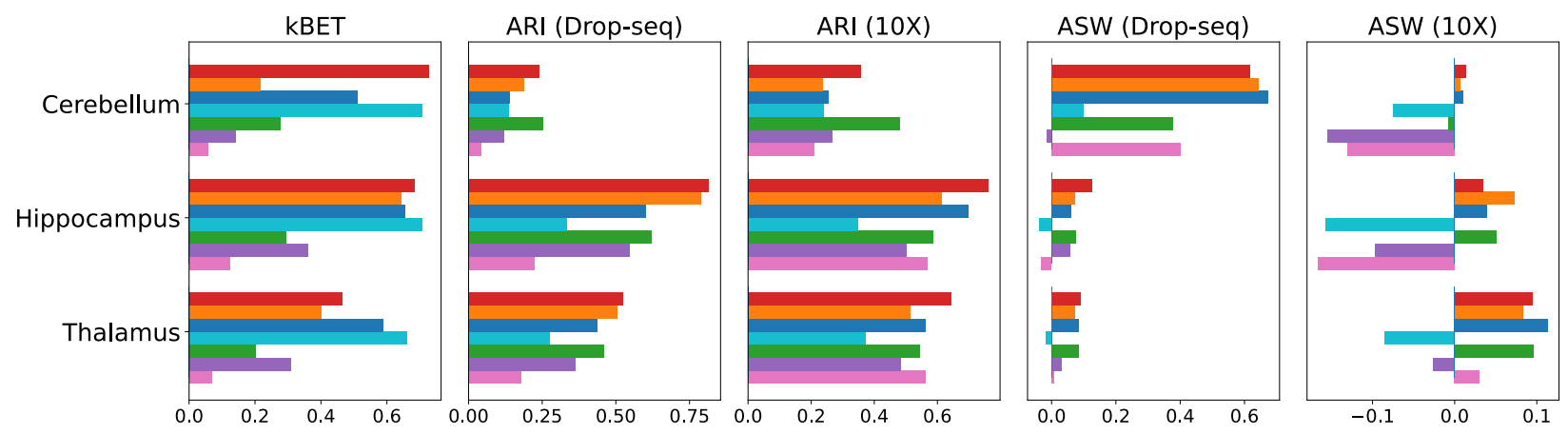

Portal Harmony

Seurat

Online iNMF

fastMNN

Scanorama

BBKNN

Figure S5: Benchmark of Portal, Harmony, Seurat, online iNMF, fastMNN, Scanorama and BBKNN. a. We evaluated running time and memory required by all compared methods. Datasets with a total sample size $n=100,000,250,000,50,000$, and 1, 100, 167 were sampled from two mouse brain atlas datasets. Considering running time and peak running memory usage, Portal was the most efficient method. Since comparison among Portal, Harmony, Seurat and online iNMF have been discussed in the main text, here we focus on investigating the performance of fastMNN, Scanorama and BBKNN. Among all compared methods, Portal and BBKNN were remarkably faster than other methods. However, BBKNN required much more memory usage than Portal as sample size increased. More importantly, BBKNN often provided less satisfactory integration performance as indicated by UMAP plots and quantitative metrics in Figs. 2, S3, S4, and (b). Similar to BBKNN, the two methods Scanorama and fastMNN also showed their comparatively limited performance compared to that of Portal, Harmony, Seurat and online iNMF. These two methods showed similar pattern of time and memory usage. Specifically, running Scanorama, fastMNN, BBKNN on full datasets with 1,100,167 cells required 33.7, 41.7, 3.5 minutes, and 15.6, 57.8, 9.3 GB respectively. As a comparison, running Portal used 2.0 minutes and 0.57 GB in the same experiment. Seurat required 24.5 GB on the datasets with 100,000 cells, so we did not include it in the comparison of peak running memory for clarity. b. Alignment (kBET) and cluster preservation performance (ARI and ASW) evaluated using datasets of three shared tissues, including cerebellum, hippocampus and thalamus, in two mouse brain atlas projects. Consistent with previous benchmarking results, fastMNN, Scanorama and BBKNN presented less accurate alignment results, indicated by low kBET, ARI and ASW scores. 

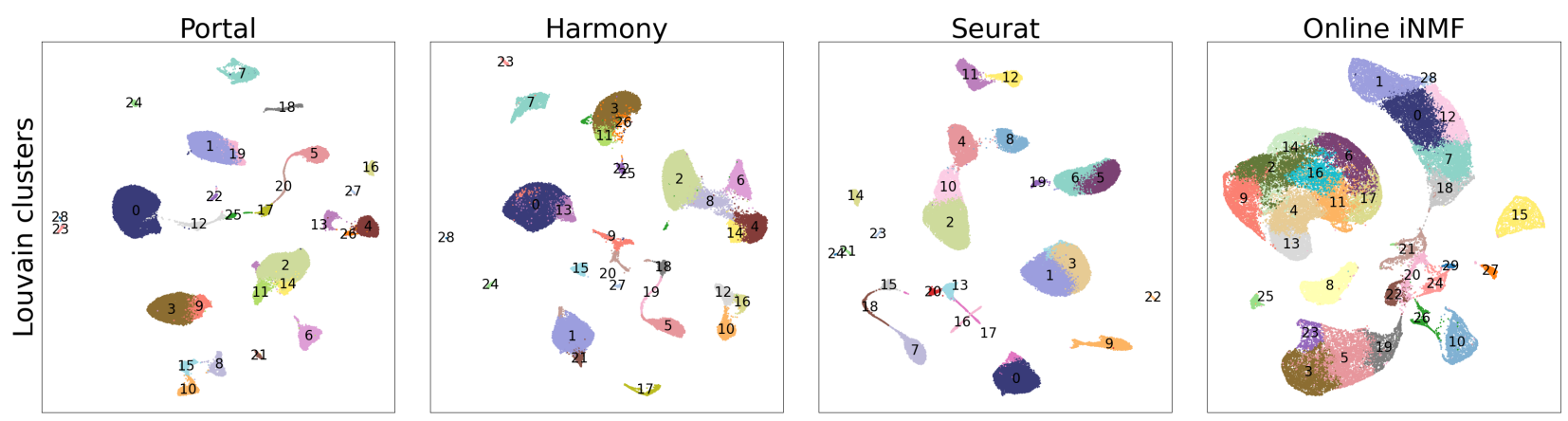

Figure S6: Clusters identified by applying the Louvain algorithm to cell embeddings obtained by Portal, Harmony, Seurat and online iNMF after integration. With default resolution setting, Louvain algorithm detected 29 (Portal), 29 (Harmony), 25 (Seurat), 30 (online iNMF) clusters as shown in UMAP plots. The UMAP plots were drawn separately and colored by clusters identified in the cell embedding space of each method, respectively.

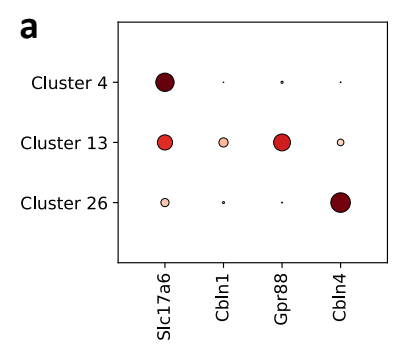

C
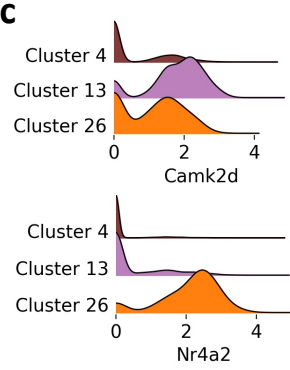

b
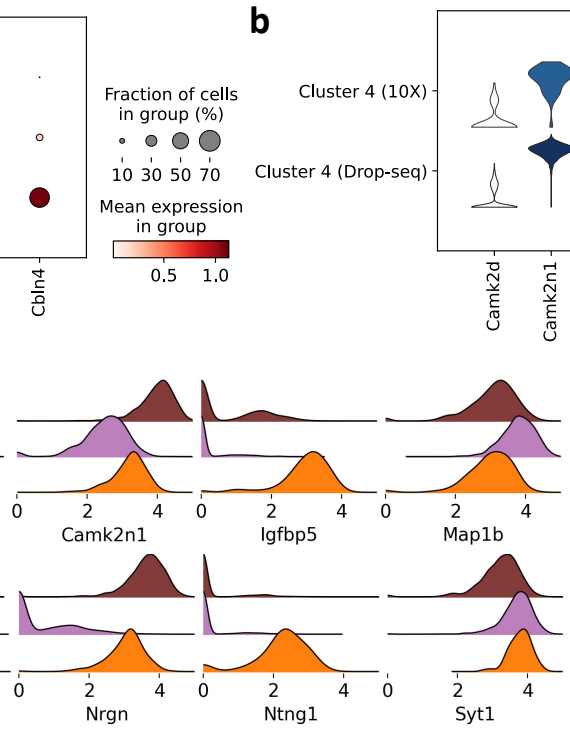

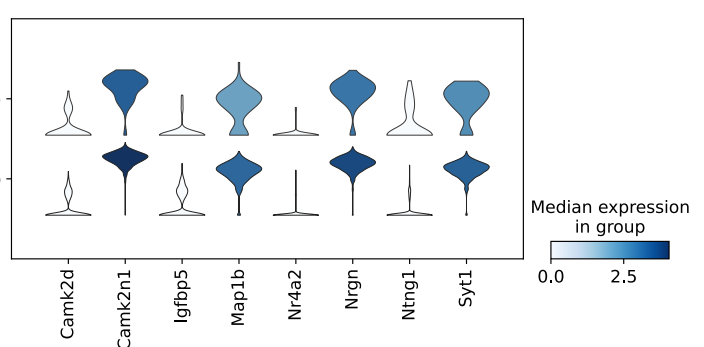

d

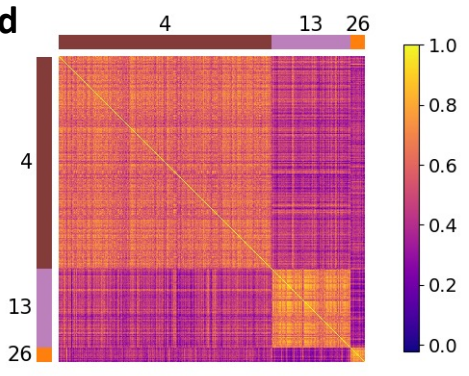

Figure S7: Detailed verification of Portal's integration result on hippocampus datasets at transcriptome level. a. We confirmed Portal's alignments of cluster 4, 13, 26 and the three neuron subpopulations by investigating the pattern of marker genes. $\mathbf{b}$. The integration result from Portal was validated by the consistent pattern of differentially expressed genes across distinct clusters. Here we only investigated into cluster 4 as cells from the 10X dataset only concentrated in cluster 4 in the marked region. c. We identified eight genes that showed distinct expression patterns across the three clusters. d. Transcriptional difference among the three clusters was further reflected by examining the correlation between cells using more genes. 

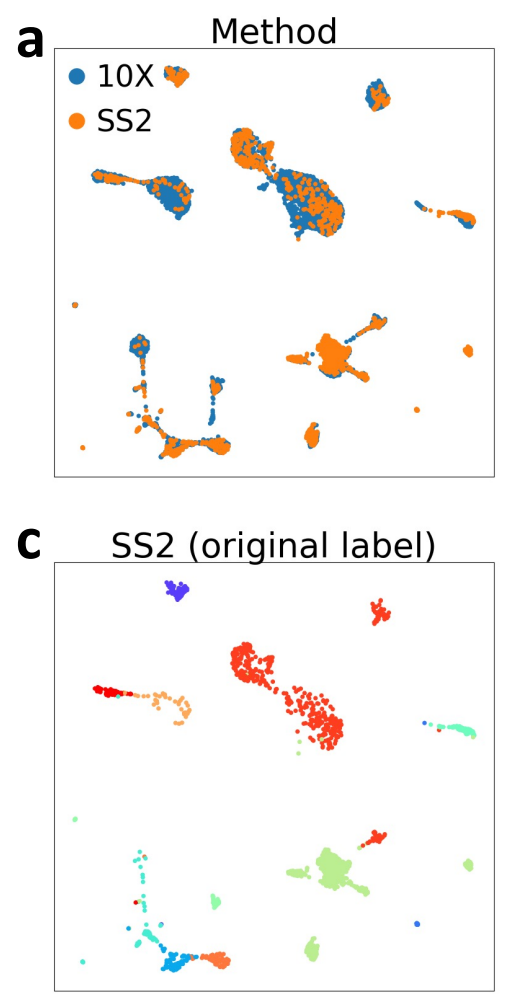
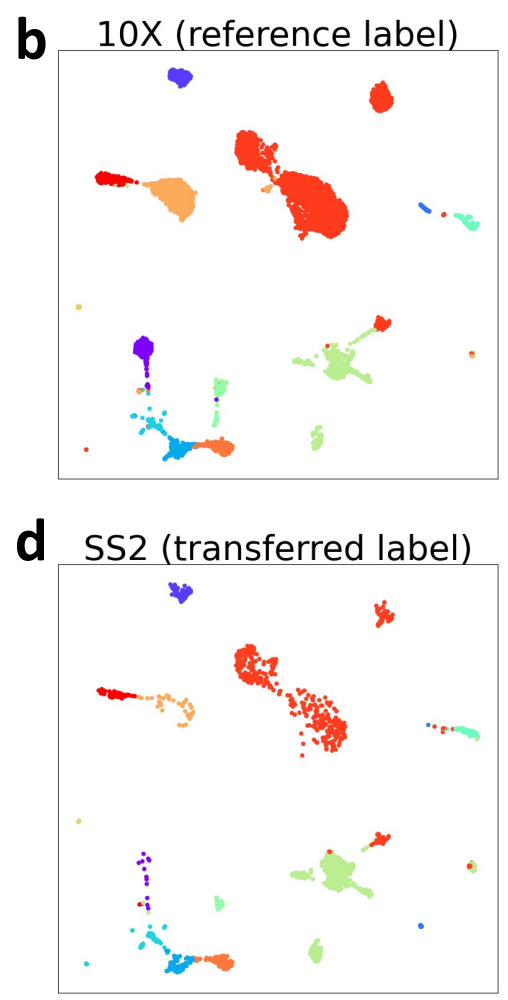

Cell type

- Alveolar macrophage

- B cell

Ciliated columnar cell of tracheobronchial tree - Classical monocyte

Dendritic cell and interstital macrophage

Dendritic cell, alveolar macrophage, and interstital macrophage

Epithelial cell of lung

Leukocyte

Lung endothelial cell

Mast cell

Natural killer cell

Non-classical monocyte

Stromal cell

T cell

Figure S8: Identification of rare subpopulations in mouse lung scRNA-seq data via label transfer. Utilizing Portal's integration result (a), we transferred annotations from the 10X dataset (b) to the SS2 dataset (d). Portal's integration helped to identify fine-grained subpopulation alveolar macrophage $(\mathbf{d})$, which was not identified in its original labels (c). a. UMAP plot of Portal's integration result colored by profiling methods. b, c. UMAP plots of integrated 10X, SS2 data colored by cell types obtained from their original publication [7]. d. UMAP plot of integrated SS2 data colored by transferred labels provided by Portal. 

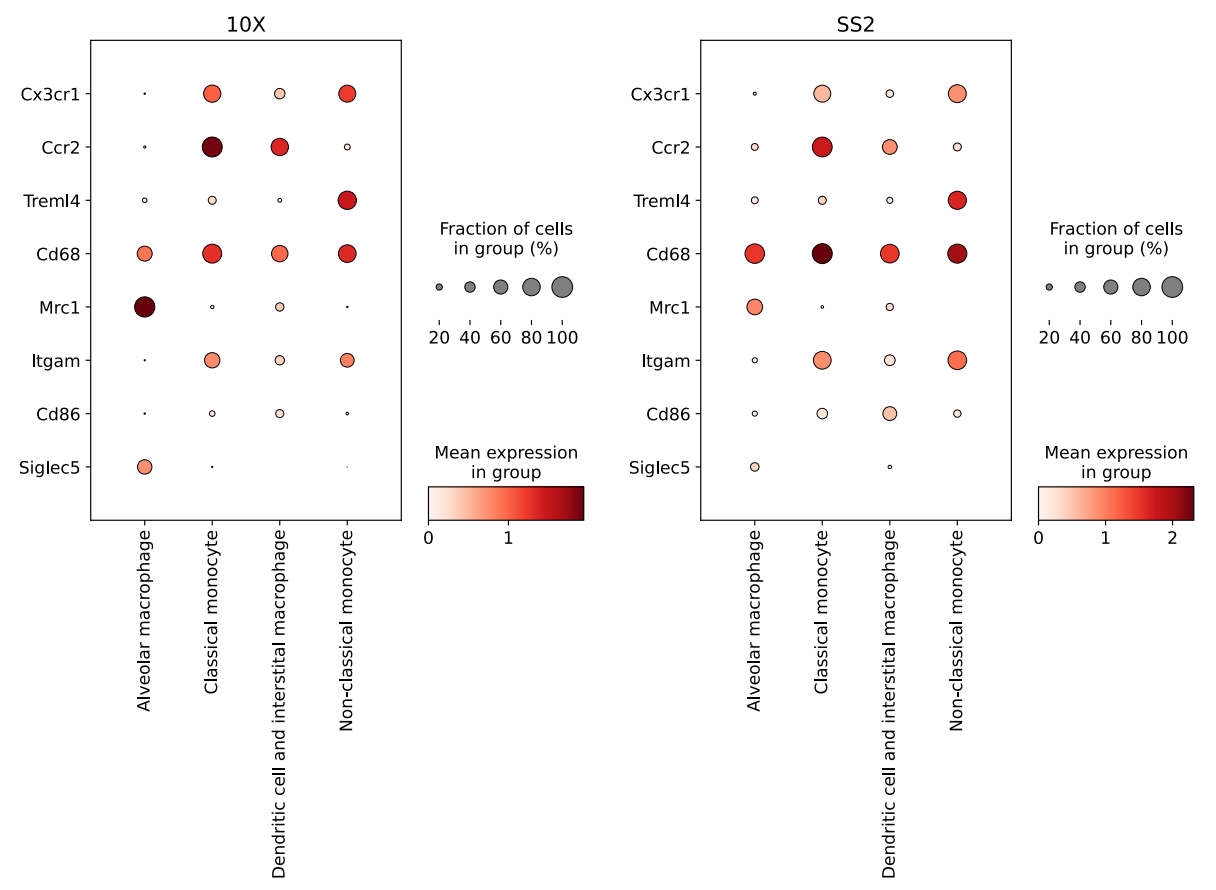

Figure S9: Marker gene pattern of identified rare subpopulations in mouse lung scRNA-seq data. By transferring labels from the 10X dataset to SS2 dataset, Portal identified four subpopulations of myeloid cells in SS2 dataset, including alveolar macrophage, dendritic cell and interstitial macrophage, classical monocyte, and non-classical monocyte. To validate the result, we examined four subpopulations' expression levels of marker genes: Cd68 is a marker of macrophages and monocytes. Between classical monocytes and non-classical monocytes, Ccr2 is a marker of classical monocytes, Cx3cr1, Treml4 are markers of non-classical monocytes. Between alveolar macrophages and interstitial macrophages, Mrc1, Siglec5 are markers of alveolar macrophages, Itgam, Cd86 are markers of interstitial macrophages. 

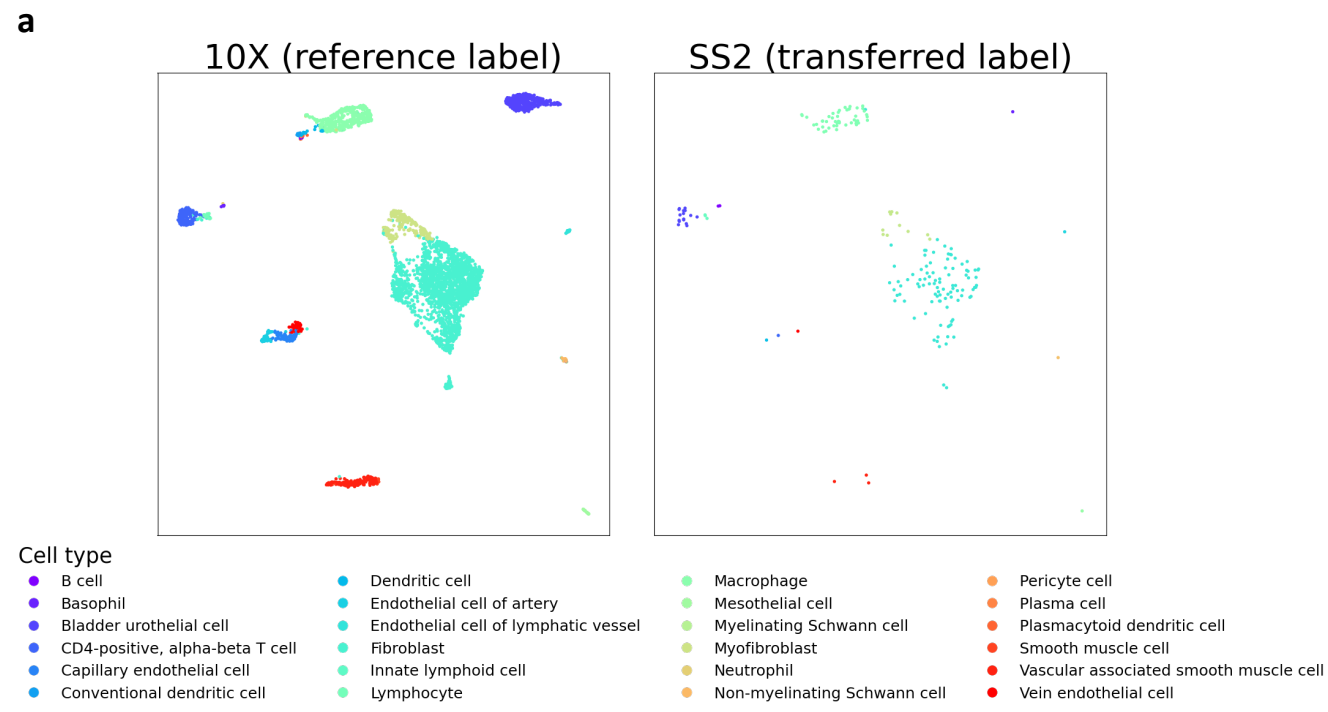

\section{b}
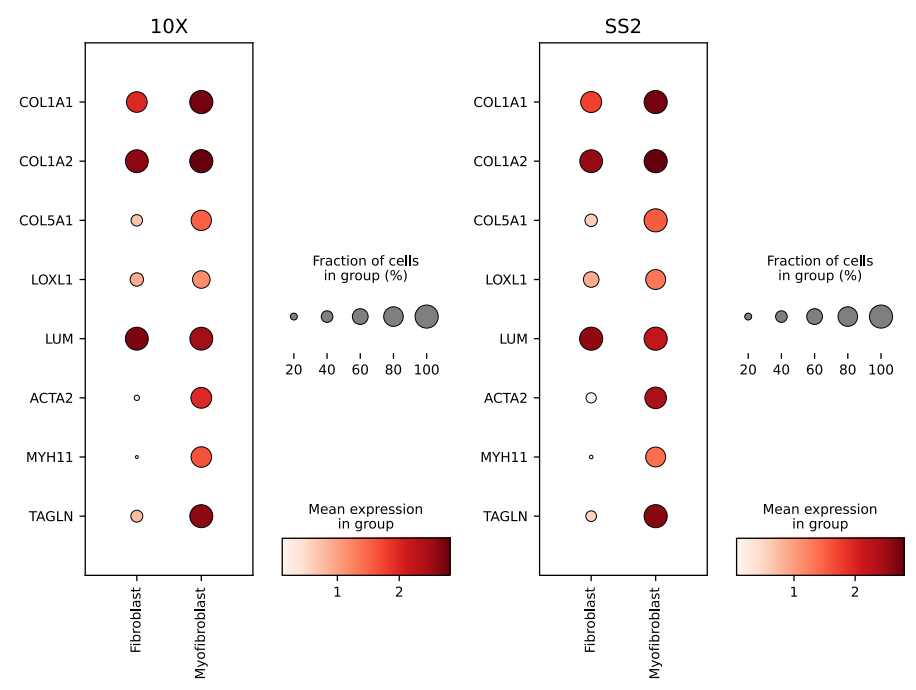

Figure S10: Identification of myofibroblast subpopulation from fibroblast population in mouse lemur bladder scRNA-seq data via label transfer. a. Portal utilized its integration result to transfer labels from the 10X dataset to the SS2 dataset. Portal successfully identified myofibroblast cells in SS2 dataset, although there were only 11 of them. b. We confirmed Portal's identification of myofibroblast cells by validating marker gene pattern. We collected eight marker genes: COL1A1, COL1A2, COL5A1, LOXL1, LUM are markers of fibroblast and myofibroblast cells. Compared to fibroblast cells, myofibroblast cells should have higher expression levels of markers ACTA2, MYH11 and TAGLN. 

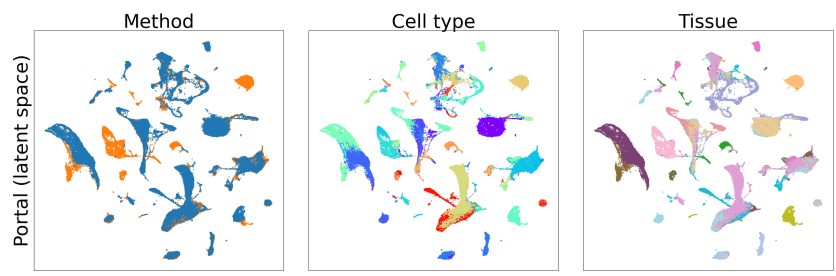

Method

$\bullet 10 \mathrm{x} \bullet \mathrm{s52}$
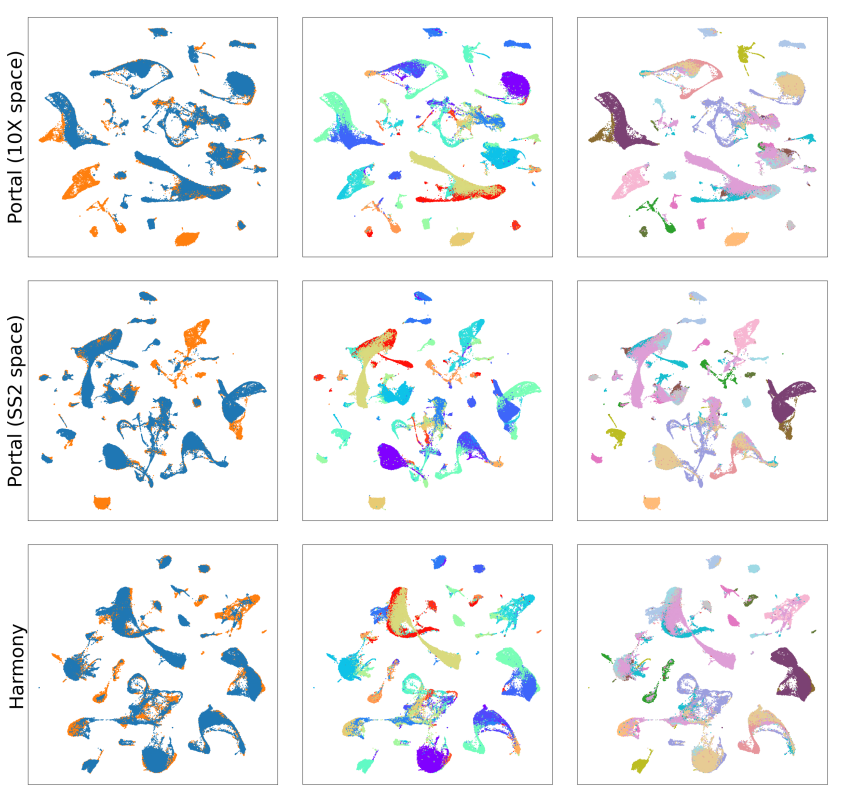

Cell type

- B cergmann glial cell

- Brush cell of epithelium proper of large intestine

kidney capillary endothelial cell

- DN1 thymic pro-T cell

- Fraction A pre-pro

- Langerhans cell

- Slamf1-negative multipotent progenitor cell

- Slamf1-positive multipotent progenitor cell

- T cell

- alveolar macrophage

- astrocyte

- basal cell of epidermis

- basophil

- bladder cell

- blood cell

- brain pericyte

- cardiac muscle cell

- cilited cosumnar cell of tracheobronchia tree

- classical monocyte

- common lymphoid progenitor

- dendritic cell

duct epithelial ce

- endocardial cell

- endocardial cell

- endothelial cell of hepatic sinusoid

- enterocyte of epithelium of large intestine

- enteroendocrine cell

- epidermal cell

- epithelial cell of large intestine

- epithelial cell of lung

- epithelial cell of proximal tubule

- erythroblast

- erythrocyte

- fibroblast

- granulocyte

- granulocytopoietic cell

- hematopoietic precursor cell
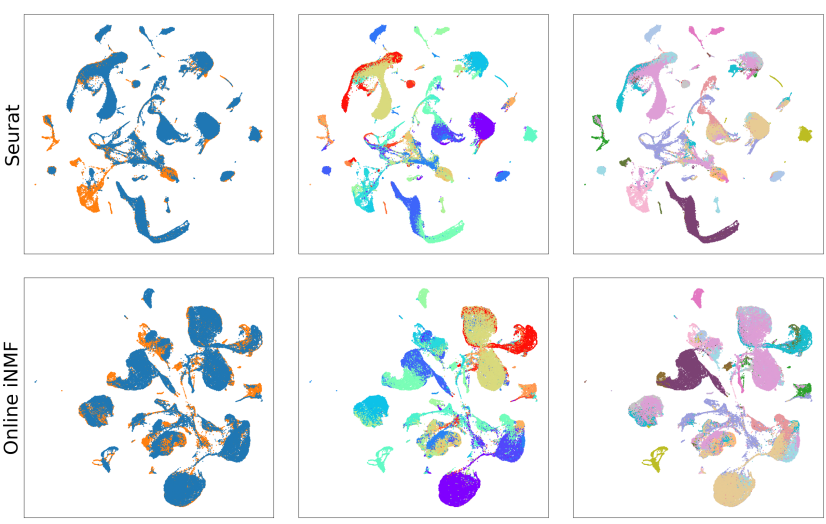

- hepatocyte

immature B cell

- immature T cell

- immature natural killer cell

- keratinocyte

- keratinocyte stem cell

(idney collecting duct epithelial cell

kidney loop of Henle ascending limb epithelial cell kidney proximal straight tubule epithelial cell - large intestine goblet cell

- late pro-B cell

- leukocyte

leukocyte
luminal epithelial cell of mammary gland

lung endothelial cell

- lymphocyte

- macrophag

- mast cell

- megakaryocyte-erythroid progenitor cell

- mesangial cell

- mesenchymal cell

- mesenchymal stem cell

- mesenchymal stem cell of adipose
mesenter

- microglial cell

- monocyte

- myeloid cell

- myofibroblast cell

- naive B cell

enatural killer cell

- neuroendocrine cell

- neuron

non-classical monocyte

- oligodendrocyte

- oligodendrocyte

- Mgodendrocyte precursor cell

- pancreatic A cell

- pancreatic D cell

- pancreatic acinar cell

- pancreatic ductal cell

- pancreatic stellate cell

- pre-natura killer cell

- precursor B cell

- proerythroblast

- professional antigen presenting cell

- promonocyte

- regulatory T cell

- skeletal muscle satellite cell

- skeletal muscle satellite stem cel

- smooth muscle cell

- stem cell of epidermis

- stromal cell

- type B pancreatic cell

Tissue

- Aorta - Liver

- Brain_Myeloid Mammary_Gland

- Brain_Non-Myeloid - Marrow

- Diaphragm - Pancreas

- Fat - Skin

- Heart Spleen

- Heart_and_Aorta $\quad$ Thymus

- Tongue
- Large_Intestine

- Limb_Muscle

Figure S11: Comparison of the capability of Portal, Harmony, Seurat and online iNMF to construct a comprehensive cell atlas across entire organism. We applied the four integration approaches to harmonize the SS2 dataset and the 10X dataset from the Tabula Muris project, where mouse cells from 20 tissues were profiled. For a comprehensive investigation into Portal's performance, we visualized integration results of Portal in three spaces, namely shared latent space, 10X data space, and SS2 data space. Notably, among the 20 tissues, only 13 of them were included in the 10X data, while all of them were included in the SS2 data. In such a integration task, Portal preserved unique cell types contained in SS2 data, e.g., microglial cells, cell types in large intestine, and pancreatic islets cells. In contrast, Harmony, Seurat and online iNMF provided less accurate results, e.g., they incorrectly mixed microglial cells in brain myeloid with macrophage cells. 

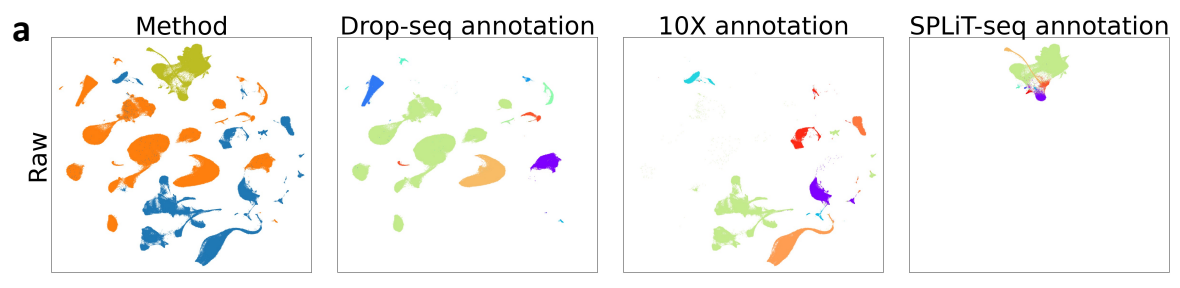
Method
Cell (Drop-seq)
Cell (10X)

Nuclei (SPLiT-seq)

b
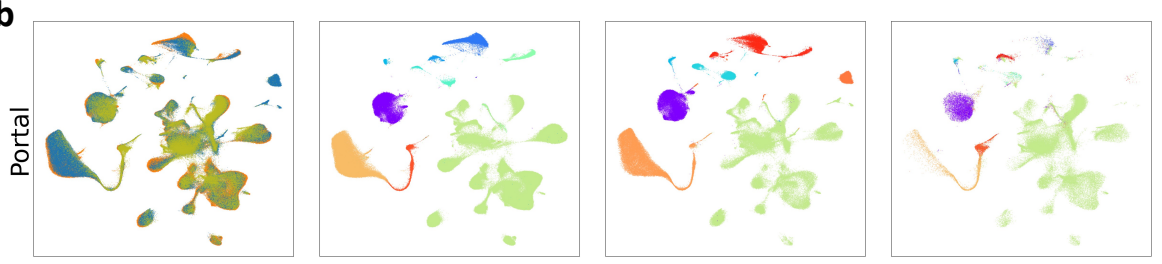

C
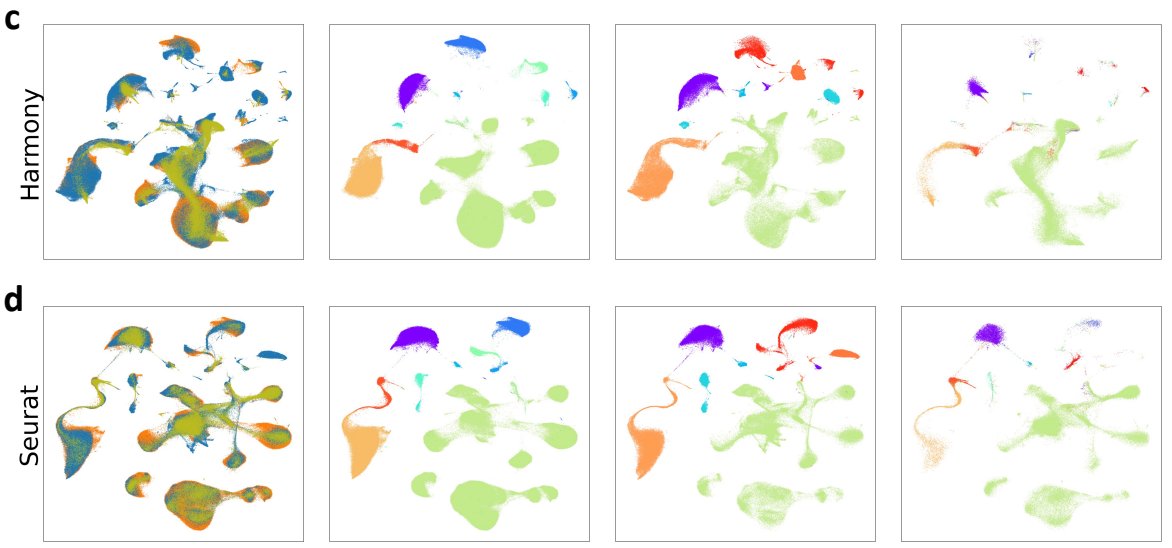

Cell type

OChoroid plexus

Ondothelial

Endothelial stalk

Endothelial tip

- Ependymal

OImmune

Macrophage

Microglia

Mitotic

Mural

Neurogenesis

Neuron

Olfactory ensheathing cell

Oligodendrocyte

Oligos

Peripheral glia

Polydendrocyte

OVascular

- Vascular and leptomeningeal cell
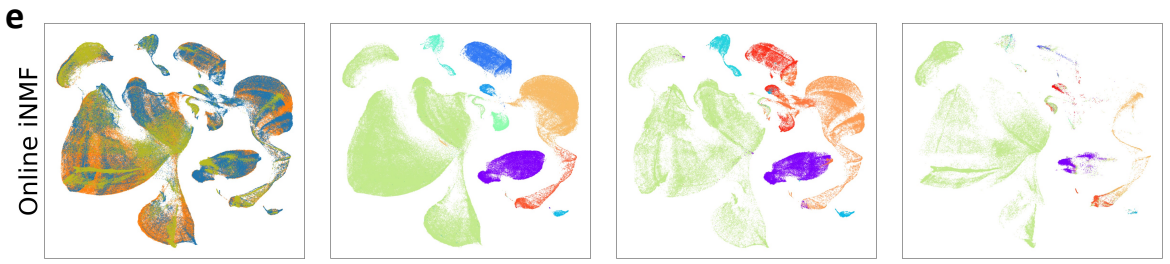

Figure S12: Comparison of the ability of Portal, Harmony, Seurat and online iNMF to build alignment across one snRNA-seq dataset and two scRNA-seq datasets. We applied the four integration approaches to align one snRNA-seq dataset profiled by SPLiT-seq [43], and two scRNA-seq datasets profiled by Drop-seq and 10X [8, 9]. We combined the cell type annotations provided by the three datasets together, although they contained slightly different annotations for non-neuron cells, e.g. immune cells and endothelial cells. a-e, UMAP visualizations of combined raw data (a), integration results of Portal (b), Harmony (c), Seurat (d) and online iNMF (e). 
bioRxiv preprint doi: https://doi.org/10.1101/2021.11.16.468892; this version posted November 19, 2021. The copyright holder for this preprint (which was not certified by peer review) is the author/funder, who has granted bioRxiv a license to display the preprint in perpetuity. It is made available under aCC-BY-NC-ND 4.0 International license.
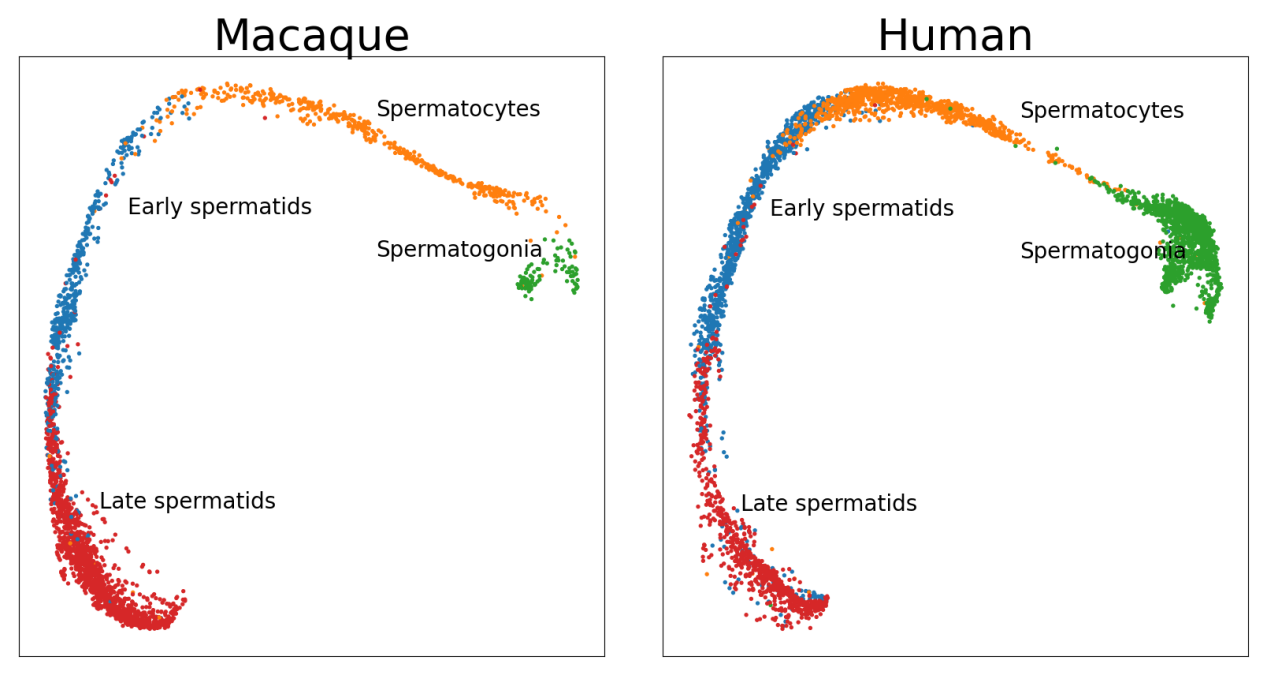

Figure S13: UMAP visualization of the original published annotations for spermatogenesis data of macaque and human [16]. 


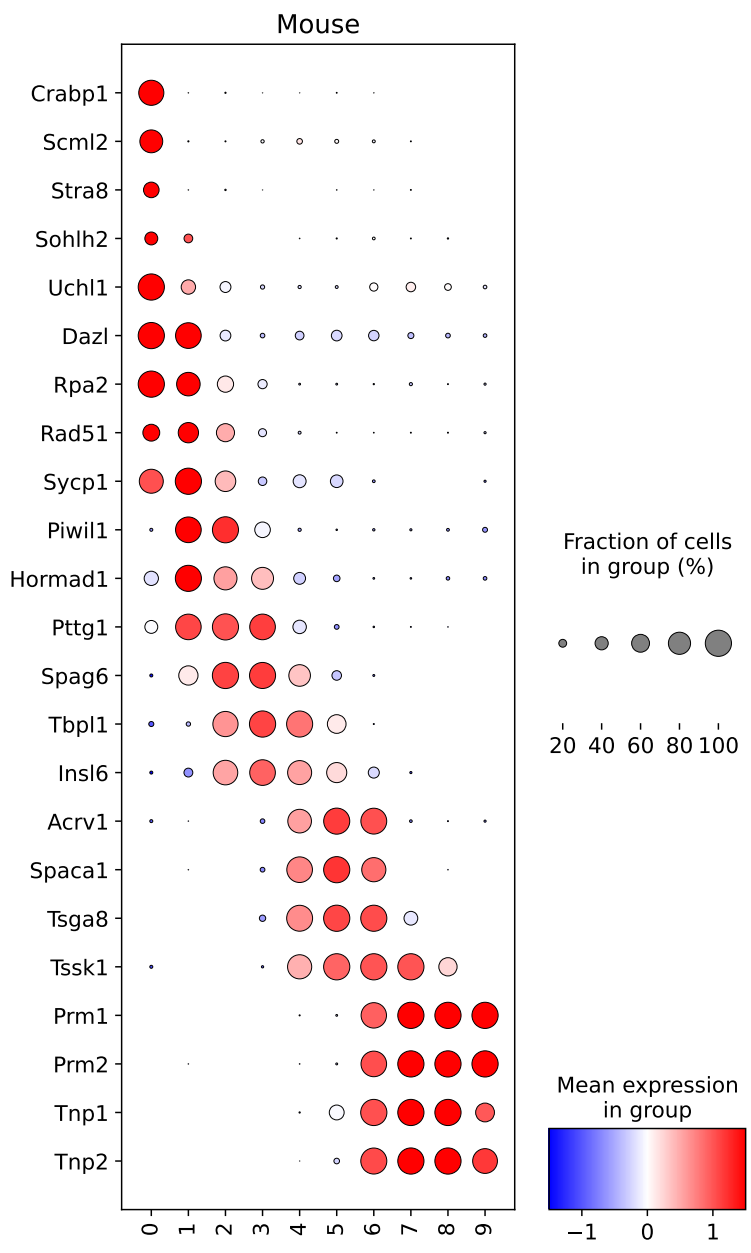

Figure S14: Marker gene patterns of mouse in Louvain clusters in cross-species integration of spermatogenesis differentiation process. For mouse, Crabp1, Scml2, Stra8, Sohlh2, Uchl, Dazl, Rpa2, Rad51, Sycp1 are markers of spermatogonia, Piwil1, Hormad1, Pttg1, Spag6, Tbpl1, Insl6 are markers of spermatocytes, Acrv1, Spaca1, Tsga8, Tssk1 are markers of early spermatids, and Prm1, Prm2, Tnp1, Tnp2 are markers of late spermatids. 

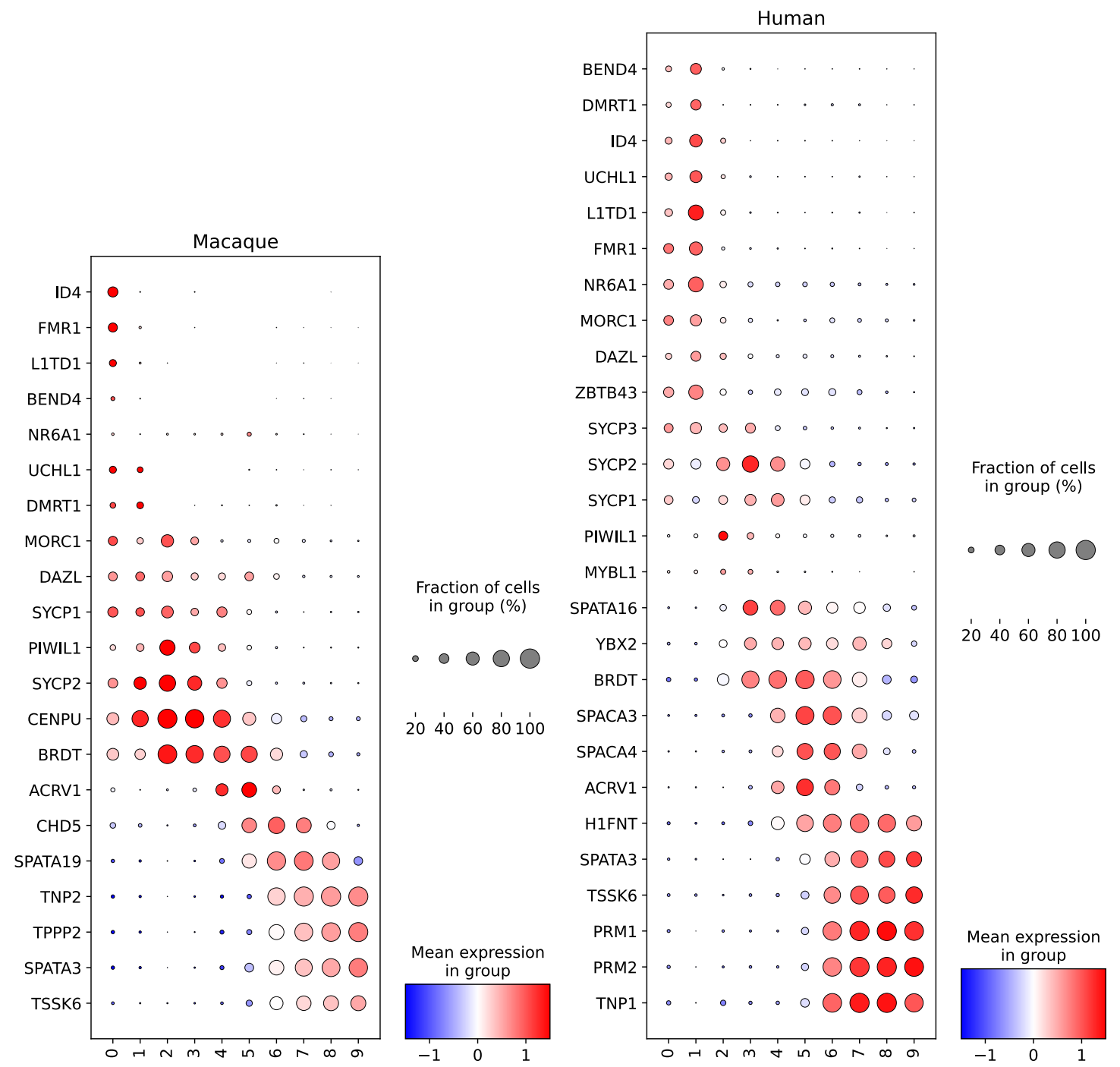

Figure S15: Marker gene patterns of macaque and human in Louvain clusters in cross-species integration of spermatogenesis differentiation process. For macaque, ID4, FMR1, L1TD1, BEND4, NR6A1, UCHL1, DMRT1, MORC1, DAZL are markers of spermatogonia, SYCP1, PIWIL1, SYCP2, CENPU are markers of spermatocytes, BRDT, ACRV1, CHD5, SPATA19 are markers of early spermatids, and TNP2, TPPP2, SPATA3, TSSK6 are markers of late spermatids. For human, BEND4, DMRT1, ID4, UCHL1, L1TD1, FMR1, NR6A1, MORC1, DAZL, ZBTB43, SYCP3 are markers of spermatogonia, SYCP2, SYCP1, PIWIL1, MYBL1, SPATA16, YBX2 are markers of spermatocytes, BRDT, SPACA3, SPACA4, ACRV1, H1FNT are markers of early spermatids, and TSSK6, PRM1, PRM2, TNP1, SPATA3 are markers of late spermatids. The marker gene patterns validated the label transfer results given by Portal. 
bioRxiv preprint doi: https://doi.org/10.1101/2021.11.16.468892; this version posted November 19, 2021. The copyright holder for this preprint (which was not certified by peer review) is the author/funder, who has granted bioRxiv a license to display the preprint in perpetuity. It is made available under aCC-BY-NC-ND 4.0 International license.
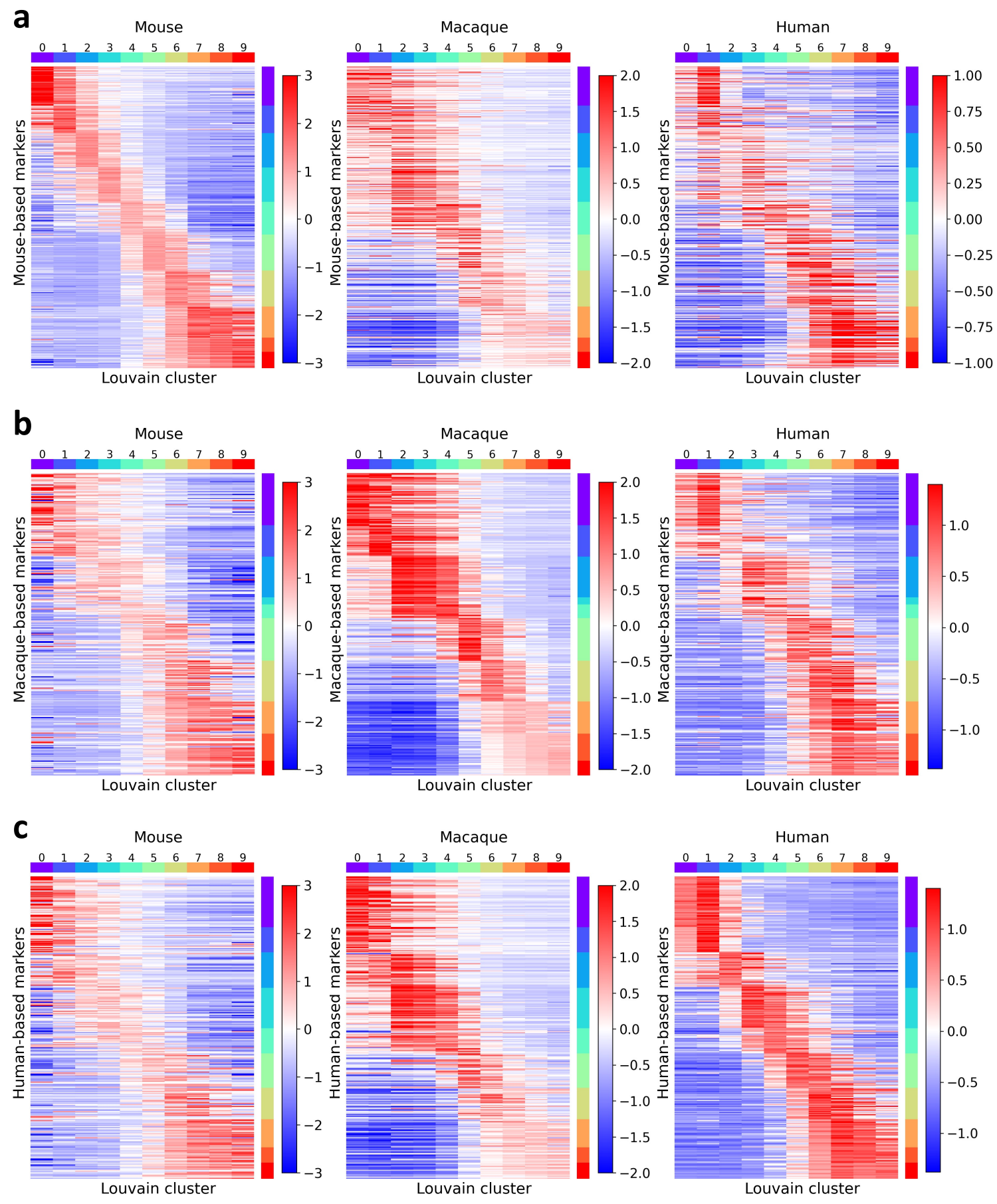

Figure S16: Gene expression heatmaps in Louvain clusters in cross-species integration of spermatogenesis differentiation process. For each species, we selected highlyexpressed genes for each cluster and combined them together. Gene expression patterns on genes selected based on mouse (a), macaque (b) and human (c) showed connection and distinction among spermatogenesis differentiation processes of different species. 